\title{
QUANTUM D-MODULES FOR TORIC NEF COMPLETE INTERSECTIONS
}

\author{
by \\ Etienne Mann \& Thierry Mignon
}

\begin{abstract}
On a smooth projective variety with $k$ ample line bundles, we denote by $Z$ the complete intersection subvariety defined by generic sections.

We define the twisted quantum $\mathcal{D}$-module which is a vector bundle with a flat connection, a flat pairing and a natural integrable structure. An appropriate quotient of it is isomorphic to the ambient part of the quantum $\mathcal{D}$-module of $Z$.

When the variety is toric, these quantum $\mathcal{D}$-modules are cyclic. The twisted quantum $\mathcal{D}$ module can be presented via mirror symmetry by the GKZ system associated to the total space of the dual of the direct sum of these line bundles.

A question is to know what is the system of equations that define the ambiant part of the quantum $\mathcal{D}$-module of $Z$. We construct this system as a quotient ideal of the GKZ system.

We also state and prove the non-equivariant twisted Gromov-Witten axioms in the appendix.
\end{abstract}

\section{Contents}

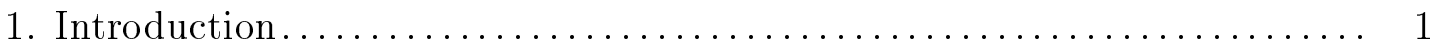

2. Twisted and reduced quantum $\mathcal{D}$-modules with geometric interpretation . 4

3. Batyrev rings for toric varieties with a splitted vector bundle........ 17

4. GKZ systems, quotient ideals and residual $\mathcal{D}$-modules.............. 29

5. Isomorphisms between quantum $\mathcal{D}$-modules and $\mathrm{GKZ}$ systems via mirror

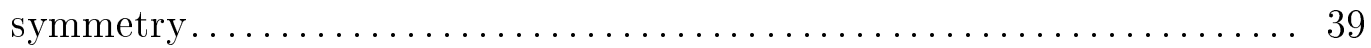

A. Twisted Axioms for Gromov-Witten invariants.................. 48

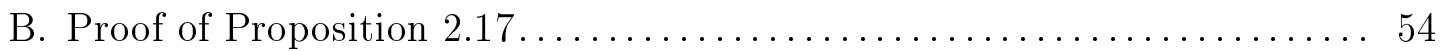

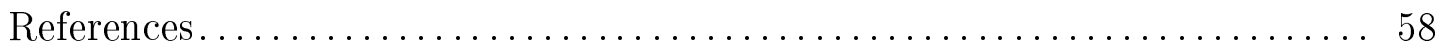

\section{Introduction}

Mirror symmetry leads to many different formulations in mathematics : equivalence of derived categories (known as Homological Mirror Symmetry by Kontsevich [Kon95]), isomorphism of Frobenius manifolds (see [Bar00]), comparison of Hodge numbers for Calabi-Yau varieties (see for example [Bat94]), isomorphism of Givental's cones (see [Giv98]), isomorphism of pure polarized TERP structures (see [Her06]) or variation of non-commutative Hodge structures (see [KKP08]), ...

2010 Mathematics Subject Classification. - 14N35, 53D45, 14F10.

Key words and phrases. - Quantum differential modules, Gromov-Witten invariants, Batyrev rings, GKZ systems, mirror symmetry, $\mathcal{D}$-modules, toric geometry.

E.M is supported by the grant of the Agence Nationale de la Recherche "New symmetries on Gromov-Witten theories" ANR- 09-JCJC-0104-01. 
Inspired by the works of Givental (see for examples [Giv96] and [Giv98]), many authors look at quantum cohomology with a differential module approach : see Kim [Kim99] for homogeneous spaces, see Coates-Corti-Lee-Tseng [CCLT06] and Guest-Sakai [GS08] for weighted projective spaces, see also the works of Iritani [Iri06], [Iri07], [Iri08] and [Iri09], the book of Cox-Katz [CK99] and the one of Guest [Gue10].

From the small quantum product on a smooth projective variety, we can define a trivial vector bundle over $H^{0}(X, \mathbb{C}) \times V \times \mathbb{C}$ where $V \subset\left(\mathbb{C}^{*}\right)^{r}$ and $r:=\operatorname{dim}_{\mathbb{C}} H^{2}(X, \mathbb{C})$ whose fiber is $H^{*}(X, \mathbb{C})$. This bundle is endowed with a flat connection and a non-degenerated pairing. This connection is sometimes called the Dubrovin-Givental connection. When $X$ is a toric smooth Fano variety, Givental (see also Iritani [Iri09] for toric weak Fano orbifolds) gives an explicit presentation of this $\mathcal{D}$-module using GKZ systems. To prove this isomorphism, he uses the equality, up to a mirror map, between the so called $I$ and $J$ functions.

In the very nice article [Iri09], Iritani enriches this quantum $\mathcal{D}$-module by adding a natural integral structure $i$.e., he defines a $\mathbb{Z}$-local system which is compatible with the connection. We call quantum $\mathcal{D}$-module, denoted by $\operatorname{QDM}(X)$, the trivial bundle endowed with a flat connection, a flat non-degenerated pairing and a natural integral structure. This $\mathbb{Z}$-local system is natural in the following sense. Assume that $X$ has a mirror (for instance $X$ is a weak Fano toric orbifolds) that is a Laurent polynomial such that its Brieskorn lattice (which is a vector bundle with a flat connection) is isomorphic to the quantum $\mathcal{D}$-module of $X$. On this Brieskorn lattice, we have a natural integral structure that comes from the Lefschetz's thimbles. The integral structure defined by Iritani is natural because it corresponds to the natural one on the mirror. Notice that the bundle, the connection, the pairing and the integral structure is part of the definition of a TERP structure defined by Hertling in [Her06] or a variation of non-commutative Hodge structure defined by Kontsevich, Katzarkov and Pantev in [KKP08].

In this paper, we investigate the same kind of objects associated to a smooth projective variety $X$ together with a splitted vector bundle $\mathcal{E}$ which is globally generated.

We use the twisted Gromov-Witten invariants and the twisted quantum products to define a trivial vector bundle, denoted by $F$, on $H^{0}(X, \mathbb{C}) \times V \times \mathbb{C}$ where $V$ is an open in $\left(\mathbb{C}^{*}\right)^{r}$ where the twisted quantum product is convergent. Inspired by the classical case, we define a flat connection $\nabla$, a flat pairing $S$ and an integral structure $F_{\mathbb{Z}}$ on it. We call twisted quantum $\mathcal{D}$-module, the quadruple $\operatorname{QDM}(X, \mathcal{E}):=\left(F, \nabla, S, F_{\mathbb{Z}}\right)$. It satisfies all the properties of the classical $\operatorname{QDM}(X)$ except that the pairing $S$ is degenerated. We quotient by the kernel of $S$ and we get a better object, called reduced quantum $\mathcal{D}$-module and denoted by $\overline{\mathrm{QDM}}(X, \mathcal{E}):=\left(\bar{F}, \bar{\nabla}, \bar{S}, \bar{F}_{\mathbb{Z}}\right)$. More precisely, we consider the trivial vector bundle $\bar{F}$ with the fibers $H^{2 *}(X, \mathbb{C}) /$ ker $m_{c_{\text {top }}}$ where $m_{c_{\text {top }}}: \alpha \rightarrow c_{\text {top }}(\mathcal{E}) \cup \alpha$ for any cohomology class $\alpha$. The data $\left(F, \nabla, S, F_{\mathbb{Z}}\right)$ pass to this quotient and we get $\overline{\mathrm{QDM}}(X, \mathcal{E})$ that satisfies all the classical properties and now $\bar{S}$ is non-degenerated. So it really looks like a quantum $\mathcal{D}$-module of a variety. Indeed, we have a geometric interpretation of $\overline{\mathrm{QDM}}(X, \mathcal{E})$ :

Theorem 1.1 (See Theorem 2.42). - Let $\mathcal{L}_{1}, \ldots, \mathcal{L}_{k}$ be ample line bundles on $X$, and assume that $\operatorname{dim}_{\mathbb{C}} X \geq k+3$. Let $Z$ be the zero of a generic section of $\mathcal{E}:=\oplus_{i=1}^{k} \mathcal{L}_{i}$. Denote by $\iota: Z \hookrightarrow X$ the closed embedding. Then the reduced quantum $\mathcal{D}$-module $\overline{\operatorname{QDM}}(X, \mathcal{E})$ is isomorphic to the sub-quantum $\mathcal{D}$-module $\mathrm{QDM}_{\text {amb }}(Z)$ of $\operatorname{QDM}(Z)$ whose fiber is $\iota^{*} H^{2 *}(X, \mathbb{C})$.

Notice that our integral structure $F_{\mathbb{Z}}$ defined on $\operatorname{QDM}(X, \mathcal{E})$ is natural because it induces the natural one on $\mathrm{QDM}_{\mathrm{amb}}(Z)$.

Then the next natural question is : can we find a presentation of $\operatorname{QDM}(X, \mathcal{E})$ and $\overline{\mathrm{QDM}}(X, \mathcal{E})$ when $X$ is a toric smooth variety in terms of GKZ systems ?

Denote by $\mathcal{D}$ the sheaf of differential operators on the basis space of the $F$ (this is not really true, the operators that we consider are $z q_{a} \partial_{q_{a}}$ where $q_{a}$ are variable in $H^{2}(X, \mathbb{C})$ and 
$z$ is the coordinate on $\mathbb{C}$ ). Denote by $Y$ the total space of the dual vector bundle $\mathcal{E}^{\vee}$. Denote by $\mathcal{G}$ the ideal sheaf associated to the GKZ system of the toric variety $Y$. We have the following result.

Theorem 1.2 (see Theorem 5.10). - Let $X$ be a smooth toric variety with $k$ line bundles $\mathcal{L}_{1}, \ldots, \mathcal{L}_{k}$ such that $\left(\omega_{X} \otimes \mathcal{L}_{1} \otimes \ldots \otimes \mathcal{L}_{k}\right)^{\vee}$ is nef. We put $\mathcal{E}:=\oplus_{i=1}^{k} \mathcal{L}_{i}$.

1. If the line bundles $\mathcal{L}_{1}, \ldots, \mathcal{L}_{k}$ are globally generated then we have the following isomorphism :

$$
\mathcal{D} / \mathcal{G} \stackrel{\sim}{\longrightarrow} \operatorname{Mir}^{*}(\mathcal{F}, \nabla)
$$

where Mir is the mirror map of Givental and $\mathcal{F}$ is the sheaf of sections of $F$.

2. If the line bundles $\mathcal{L}_{1}, \ldots, \mathcal{L}_{k}$ are ample, we have the following commutative diagram

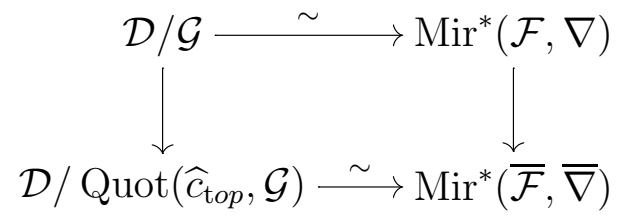

where $\widehat{c}_{\text {top }}$ is an operator attach to the cohomology class $c_{\text {top }}(\mathcal{E})$ (cf. Notation 4.1) and $\operatorname{Quot}\left(\widehat{c}_{\text {top }}, \mathcal{G}\right)$ is the left quotient ideal $\left\langle P \in \mathcal{D}, \widehat{c}_{\text {top }} P \in \mathcal{G}\right\rangle$.

Notice that, unlike the commutative case, the set $\left\{P \in \mathcal{D}, \widehat{c}_{\text {top }} P \in \mathcal{G}\right\}$ is not an ideal.

The ideal sheaf Quot $\left(\widehat{c}_{\text {top }}, \mathcal{G}\right)$ answer to the following question which is addressed in the [CK99, p.94-95 and p.101]: What differential equations shall we add to $\mathcal{G}$ to get an isomorphism with $\mathrm{QDM}_{\mathrm{amb}}(Z)$ ?

The isomorphisms above are based on the equality (up to the mirror map) between the twisted $J$-function and the twisted $I$-function of Givental (see [Giv96] and [Giv98]) and a careful analysis of the local freeness and rank of GKZ modules. Freeness and rank requires the study of Batyrev rings of the toric variety $Y$-the total space of $\mathcal{E}^{\vee}$ - which will appear as the restriction of the $\mathcal{D}$-modules at $z=0$, and can be thought as a twisted Batyrev ring of the pair $(X, \mathcal{E})$.

Proving this theorem leads to develop quite a lot of materials and results which deserve some precisions. Let us sketch our strategy of proof.

For the first point of the theorem above, we show that $\mathcal{D} / \mathcal{G}$ is a locally free sheaf of rank $\operatorname{dim}_{\mathbb{C}} H^{2 *}(X, \mathbb{C})=\operatorname{rk} F$ (see Theorem 4.10). This is done in 2 steps.

- We first prove the coherence of $\mathcal{D} / \mathcal{G}$ (see Theorem 4.5). This implies the local freeness over $z \neq 0$ and we use Adolphson's result in [Ado94] to compute the rank.

- On $z=0$, we have a tautological isomorphism between $\mathcal{D} /\left.\mathcal{G}\right|_{z=0}$ and the Batyrev ring of $Y$. We prove that this ring is locally free of $\operatorname{rank}$ rk $F$ over a suitable algebraic neighborhood $\bar{U}$ (see below).

For second point of the theorem above, we show in Theorem 4.14 :

- On $z=0$, we prove that the natural morphism between $\mathcal{D} /\left.\operatorname{Quot}\left(\widehat{c}_{\text {top }}, \mathcal{G}\right)\right|_{z=0}$ and the residual Batyrev ring (see Definition 3.39) of $Y$ is an isomorphism. We prove that this residual ring is locally free of $\operatorname{rank} \operatorname{rk} \bar{F}=\operatorname{dim} H^{2 *}(X)-\operatorname{dim} \operatorname{ker} m_{c_{t o p}}$ over $\bar{U}$.

- on $z \neq 0$ the coherence of $\mathcal{D} / \mathcal{G}$ implies that $\mathcal{D} / \operatorname{Quot}\left(\widehat{c}_{\text {top }}, \mathcal{G}\right)$ is locally free of rank less than $\operatorname{rk} \bar{F}$.

Let us collect the precise results that we prove on the Batyrev rings, which are interesting on their own :

Theorem 1.3. - Let $X$ be a smooth toric variety with $k$ globally generated line bundles $\mathcal{L}_{1}, \ldots, \mathcal{L}_{k}$ such that the total space of the vector bundle $\mathcal{E}:=\bigoplus_{i=1}^{k} \mathcal{L}_{i}$ has a nef anticanonical 
divisor. Denote by $\bar{U}$ the good neighborhood in the spectrum of the Novikov ring defined in Notation 3.34.

1. (See Theorem 3.26) Denote by B the Batyrev ring (see Definition 3.12) of the total space of $\mathcal{E}^{\vee}$.The morphism : $\left.\operatorname{Spec}(B)\right|_{\bar{U}} \longrightarrow \bar{U}$ is finite, flat, of degree $\operatorname{dim} H^{2 *}(X, \mathbb{C})$.

2. (See Proposition 3.40) Moreover, if the line bundles $\mathcal{L}_{1}, \ldots, \mathcal{L}_{k}$ are ample then the morphism : $\left.\operatorname{Spec}\left(B^{\mathrm{res}}\right)\right|_{\bar{U}} \longrightarrow \bar{U}$ is finite, flat, of degree $\operatorname{dim} \overline{H^{2 *}(X, \mathbb{C})}$ where $B^{\mathrm{res}}$ is the residual Batyrev ring (see Definition 3.39).

The plan of this article is the following.

In Section 2, we define first (Subsection 2.1) the twisted quantum $\mathcal{D}$-module $\operatorname{QDM}(X, \mathcal{E})$ with all its properties and its natural integral structure. Then we define the reduced quantum $\mathcal{D}$-module $\overline{\mathrm{QDM}}(X, \mathcal{E})$ in Subsection 2.2. Finally, we give the geometric interpretation in Subsection 2.3 where we prove the first Theorem 1.1.

In Section 3, we focus on Batyrev rings for toric varieties. Notice that this section can be read independently of the rest of the paper. The first Subsection 3.1 is devoted to some recalls on toric geometry. In Subsection 3.1 we construct the fan of the total space of the vector bundle $\mathcal{E}$. In Subsection 3.2, we define the Batyrev rings. Subsection 3.3 is devoted to some recalls on the primitive collections. In Subsection 3.4, we prove that the quantum Stanley-Reisner ideal has a Groebner basis indexed by primitive collections (See Theorem 3.22). In Subsections 3.5 and 3.6, we prove the Theorem 3.26 and Proposition 3.40 quoted above in Theorem 1.3.

In Section 4, we focus on GKZ modules. We prove first that the GKZ module $\mathcal{D} / \mathcal{G}$ is coherent in Theorem 4.5. Then we prove that it is locally free of rank rk $F$ in Theorem 4.10. We finish by a result on the residual GKZ module $\mathcal{D} / \operatorname{Quot}\left(\widehat{c}_{\text {top }}, \mathcal{G}\right)$ (see Theorem 4.14). These results use Theorem 3.26 and Proposition 3.40 of the previous section.

In Section 5, we start by some recall on Givental's mirror symmetry in Subsection 5.1 then we state and prove Theorem 1.2 in Subsection 5.2.

We finish this paper by two appendices. Appendix A contains the proof of the twisted Gromov-Witten invariants in genus 0 that are known from the experts. We add it by lack of references.

Appendix $\mathrm{B}$ is a complete proof of the flatness of the connection $\nabla$ using the twisted axioms.

Acknowledgment : We thank Thomas Reichelt, Claude Sabbah and Christian Sevenheck for useful discussions. The seminar in Paris organized by Serguei Barannikov and Claude Sabbah on the non-commutative Hodge structures was the starting point of this paper. We also thank Antoine Douai for helping in the organization of the workshop in Luminy on the work of Iritani. We are also grateful to Hiroshi Iritani that pointed out the reference [Mav00] (see Remark 2.40) The first author is supported by the ANR New symetries in Gromov-Witten theories number ANR- 09-JCJC-0104-01.

Notation 1.4. - We use calligraphic letters for the sheaves like $\mathcal{M}, \mathcal{M}^{\text {res }}, \mathcal{G}, \mathcal{B}, \mathcal{L}, \mathcal{E}$. We use bold letters for modules or ideals on non commutative rings $\mathbb{M}, \mathbb{M}^{\text {res }}, \mathbb{G}, \mathbb{A}, \ldots$

\section{Twisted and reduced quantum $\mathcal{D}$-modules with geometric interpretation}

Let $X$ be a smooth projective complex variety of dimension $n$ and $k$ globally generated line bundles $\mathcal{L}_{1}, \ldots, \mathcal{L}_{k}$. Denote by $\mathcal{E}$ the $\operatorname{sum} \mathcal{E}:=\mathcal{L}_{1} \oplus \cdots \oplus \mathcal{L}_{k}$.

We first define the twisted quantum $\mathcal{D}$-module, denoted by $\operatorname{QDM}(X, \mathcal{E})$, associated to these data (Definition 2.24). This is a trivial bundle of rank $\operatorname{dim}_{\mathbb{C}} H^{2 *}(X, \mathbb{C})$ with an integrable connection, a flat pairing and an integral structure. 
It turns out that the pairing of the twisted quantum $\mathcal{D}$-module is degenerated, which makes $\operatorname{QDM}(X, \mathcal{E})$ a not so natural object, without clear geometric meaning. In a second paragraph we introduce the reduced quantum $\mathcal{D}$-module $\overline{\mathrm{QDM}}(X, \mathcal{E})$ (Definition 2.34 ) ; it is constructed as the quotient of $\operatorname{QDM}(X, \mathcal{E})$ by the kernel of the endomorphism $m_{c_{\text {top }}}$, which is the cup multiplication by the Euler class $c_{\text {top }}(\mathcal{E})$ of $\mathcal{E}$ :

$$
\begin{aligned}
m_{c_{\text {top }}}: H^{2 *}(X, \mathbb{C}) & \longrightarrow H^{2 *}(X, \mathbb{C}) \\
\alpha & \longmapsto \alpha \cup c_{\text {top }}(\mathcal{E}) .
\end{aligned}
$$

The reduced quantum $\mathcal{D}$-module is a trivial bundle of $\operatorname{rank} \operatorname{dim} H^{2 *}(X, \mathbb{C})-\operatorname{dim} \operatorname{ker} m_{\text {ctop }}$ with an integrable connection, a flat non-degenerated pairing and an integral structure.

If $\operatorname{dim} X \geq k+3$, we also consider a generic section of $\mathcal{E}$ and denote by $Z$ the complete intersection subvariety defined as the zero locus of this section. By Bertini's theorem over $\mathbb{C}$, the subvariety $Z$ is smooth and connected. Assuming moreover that the $\mathcal{L}_{i}$ are ample line bundles, the Lefschetz theorem gives an isomorphism between $H^{2}(X, \mathbb{C})$ and $H^{2}(Z, \mathbb{C})$.

We can compare $\operatorname{QDM}(X, \mathcal{E}), \overline{\mathrm{QDM}}(X, \mathcal{E})$ and the classical, untwisted, quantum $\mathcal{D}$ module of $Z, \operatorname{QDM}(Z)$. This will be made in the last subsection.

Notation 2.1. - For $0 \leq i \leq 2 n$, denote by $H^{i}(X):=H^{i}(X, \mathbb{C})$ the complex cohomology group of classes of degree $i$. Also denote by $H^{*}(X)$ the complex cohomology ring $\oplus_{i=0}^{2 n} H^{i}(X)$; the even part of this ring will be written $H^{2 *}(X)$. Put $s=\operatorname{dim}_{\mathbb{C}} H^{2 *}(X)$ and $r=\operatorname{dim}_{\mathbb{C}} H^{2}(X)$.

We fix, once and for all, a homogeneous basis $\left(T_{0}, \ldots, T_{s-1}\right)$ of $H^{2 *}(X)$ such that $T_{0}=\mathbf{1}$ is the unit for the cup product and that the classes $T_{1}, \ldots, T_{r}$ form a basis of $H^{2}(X, \mathbb{Z})$ modulo torsion. Denote by $\left(t_{0}, \ldots, t_{s-1}\right)$ the associated coordinates and put $\tau:=\sum_{a=0}^{s-1} t_{a} T_{a}$ and $\tau_{2}:=$ $\sum_{a=1}^{r} t_{a} T_{a}$. Also denote by $\left(T^{0}, \ldots, T^{s-1}\right)$ the Poincaré dual in $H^{2 *}(X)$ of $\left(T_{0}, \ldots, T_{s-1}\right)$.

As a convention, We will write $H_{2}(X, \mathbb{Z})$ for the degree 2 integer homology modulo torsion. Denote by $\left(B_{1}, \ldots, B_{r}\right)$ the dual basis of $\left(T_{1}, \ldots, T_{r}\right)$ in $H_{2}(X, \mathbb{Z})$. The associated coordinates will be denoted by $\left(d_{1}, \ldots, d_{r}\right)$.

We denote by $\mathcal{T}_{X}$ the tangent bundle of $X, \omega_{X}$ the canonical sheaf, and fix a canonical divisor $K_{X}$.

As a convention, we will make no notational distinction between vector bundles and locally free sheaves, writing -for example- $\mathcal{E}$ and $\mathcal{L}_{i}$ for both.

2.1. Twisted quantum $\mathcal{D}$-module. - In this subsection, we define the twisted quantum $\mathcal{D}$-module $\operatorname{QDM}(X, \mathcal{E})=\left(F, \nabla, S, F_{\mathbb{Z}}\right)$.

2.1.a. Twisted quantum product. - First recall the definition of the twisted Gromov-Witten invariant ( $c f$. [Giv96] and [CG07] or [CK99, Section 11.2.1] and [Pan98]).

Let $\ell$ be in $\mathbb{N}$ and $d$ be in $H_{2}(X, \mathbb{Z})$. Denote by $X_{0, \ell, d}$ the moduli space of stable maps of degree $d$ from rational curves with $\ell$ marked points to $X$. The universal curve over $X_{0, \ell, d}$ is $X_{0, \ell+1, d}$ :

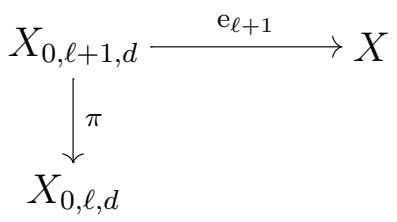

where $\pi$ is the map that forgets the $(\ell+1)$-th point and stabilizes, and $\mathrm{e}_{\ell+1}$ is the evaluation at the $(\ell+1)$-th marked point.

Recall that a convex bundle $\mathcal{N}$ on $X$ is a vector bundle such that, for any stable map $f: C \rightarrow X$ where $C$ is a rational nodal curve, $H^{1}\left(C, f^{*} \mathcal{N}\right)=0$. 
Proposition 2.2. - Let $\mathcal{N}$ be a globally generated vector bundle (not necessarily splitted) of rank $b$ then $\mathcal{N}$ is convex and the sheaf $\mathcal{N}_{0, \ell, d}:=R^{0} \pi_{*} \mathrm{e}_{\ell+1}^{*} \mathcal{N}$ is locally free of rank $\int_{d} c_{1}(\mathcal{N})+b$.

Proof. - Let us prove the convexity of $\mathcal{N}$. We follow [FP97, Lemma 10].

Let $f: C \longrightarrow X$ be a stable map and $p$ be a non singular point on $C$. We will prove by induction on the number of irreducible components of $C$ that

$$
H^{1}\left(C, f^{*} \mathcal{N} \otimes \mathcal{O}_{C}(-p)\right)=0 .
$$

First, assume that $C \simeq \mathbb{P}^{1}$. We can write $f^{*}(\mathcal{N}) \simeq \oplus_{i=1}^{b} \mathcal{O}_{\mathbb{P}^{1}}\left(a_{i}\right)$ with $a_{1}, \ldots, a_{b}$ in $\mathbb{Z}$. Since $\mathcal{N}$ is globally generated, $f^{*}(\mathcal{N})$ also is, which implies $a_{i} \geq 0$ for any $i$ in $\{1, \ldots, b\}$. It follows that $H^{1}\left(\mathbb{P}^{1}, f^{*} \mathcal{N} \otimes \mathcal{O}_{C}(-p)\right)=\oplus_{i=1}^{b} H^{1}\left(\mathbb{P}^{1}, \mathcal{O}_{\mathbb{P}^{1}}\left(a_{i}-1\right)\right)=0$.

Assume now that $C=C^{\prime} \cup C_{0}$ where $C_{0} \simeq \mathbb{P}^{1}$ and $p$ in $C_{0}$. Denote by $p_{1}, \ldots, p_{q}$ the points of $C_{0} \cap C^{\prime}$. Notice that $C^{\prime}$ has exactly $q$ connected components intersecting $C_{0}$ on exactly one point. Each $p_{i}$ is a smooth point of one of these components. We have the following exact sequence

$$
0 \longrightarrow f^{*} \mathcal{N} \otimes \mathcal{O}_{C^{\prime}}\left(-\sum_{i=1}^{q} p_{i}\right) \longrightarrow f^{*} \mathcal{N} \otimes \mathcal{O}_{C}(-p) \longrightarrow f^{*} \mathcal{N} \otimes \mathcal{O}_{C_{0}}(-p) \longrightarrow 0
$$

From the associated long exact sequence and by the inductive assumption on the connected components of $C^{\prime}$, we deduce the equality (2.3). The exact sequence :

$$
0 \longrightarrow f^{*} \mathcal{N} \otimes \mathcal{O}_{C}(-p) \longrightarrow f^{*} \mathcal{N} \longrightarrow f^{*} \mathcal{N} \otimes \mathcal{O}_{p} \longrightarrow 0
$$

gives $H^{1}\left(C, f^{*} \mathcal{N}\right)=0$.

Now, the stalk at a point $\left(C, x_{1}, \ldots, x_{\ell}, f: C \rightarrow X\right)$ in $X_{0, \ell, d}$ of the K-theoretic pushforward $\mathcal{N}_{0, \ell, d}$ is $H^{0}\left(C, f^{*} \mathcal{N}\right)-H^{1}\left(C, f^{*} \mathcal{N}\right)$. Since $\mathcal{N}$ is convex $H^{1}\left(C, f^{*} \mathcal{N}\right)=0$ and $H^{0}\left(C, f^{*} \mathcal{N}\right)$ has dimension $\int_{d} c_{1}(\mathcal{N})+b$ by Riemann-Roch. Thus, $\mathcal{N}_{0, \ell, d}$ is locally free of dimension $\int_{d} c_{1}(\mathcal{N})+b$ on $X_{0, \ell, d}$.

Let $\mathcal{E}_{0, \ell, d}$ be the sheaf $R^{0} \pi_{*} \mathrm{e}_{\ell+1}^{*} \mathcal{E}$ as in Proposition 2.2. For $j$ in $\{1, \ldots, \ell\}$, we define the surjective morphism $\mathcal{E}_{0, \ell, d} \rightarrow \mathrm{e}_{j}^{*} \mathcal{E}$ by evaluating the section to the $j$-th marked point. We define $\mathcal{E}_{0, \ell, d}(j)$ to be the kernel of this map that is we have the following exact sequence

$$
0 \longrightarrow \mathcal{E}_{0, \ell, d}(j) \longrightarrow \mathcal{E}_{0, \ell, d} \longrightarrow \mathrm{e}_{j}^{*} \mathcal{E} \longrightarrow 0
$$

By Proposition 2.2, for any $j \in\{1, \ldots, \ell\}$ the bundle $\mathcal{E}_{0, \ell, d}(j)$ has $\operatorname{rank} \int_{d} c_{1}(\mathcal{E})$.

For $i \in\{1, \ldots, \ell\}$, let $\mathfrak{N}_{i}$ be the line bundle on $X_{0, \ell, d}$ whose fiber at a point $\left(C, x_{1}, \ldots, x_{\ell}\right.$, $f: C \rightarrow X)$ is the cotangent space $\mathcal{T}^{*} C_{x_{i}}$. Put $\psi_{i}:=c_{1}\left(\mathfrak{N}_{i}\right)$ in $H^{2}\left(X_{0, \ell, d}\right)$.

Definition 2.5. - Let $\ell$ be in $\mathbb{N}, \gamma_{1}, \ldots, \gamma_{\ell}$ be classes in $H^{2 *}(X), d$ be in $H_{2}(X, \mathbb{Z})$ and $\left(m_{1}, \ldots, m_{\ell}\right)$ be in $\mathbb{N}^{\ell}$. For $j$ in $\{1, \ldots, \ell\}$, the $(j$-th) twisted Gromov-Witten invariant with descendants of these data is defined and denoted by

$$
\left\langle\tau_{m_{1}}\left(\gamma_{1}\right), \ldots, \widetilde{\tau_{m_{j}}\left(\gamma_{j}\right)}, \ldots, \tau_{m_{\ell}}\left(\gamma_{\ell}\right)\right\rangle_{0, \ell, d}:=\int_{\left[X_{0, \ell, d}\right]^{\text {vir }}} c_{\mathrm{top}}\left(\mathcal{E}_{0, \ell, d}(j)\right) \prod_{i=1}^{\ell} \psi_{i}^{m_{i}} \mathrm{e}_{i}^{*} \gamma_{i}
$$

where $\mathrm{e}_{i}: X_{0, \ell, d} \rightarrow X(1 \leq i \leq \ell)$ is the evaluation morphism to the $i$ th marked point and $\left[X_{0, \ell, d}\right]^{\mathrm{vir}}$ is the virtual class on $X_{0, \ell, d}$.

Definition 2.6. - Let $\tau_{2}$ be a class of $H^{2}(X)$ and $\gamma_{1}, \gamma_{2}$ be in $H^{2 *}(X)$. The twisted small quantum product (with respect to $\mathcal{E}$ ) of $\gamma_{1}$ and $\gamma_{2}$ is defined by

$$
\gamma_{1} \bullet \bullet_{\tau_{2}}^{\mathrm{tw}} \gamma_{2}:=\sum_{a=0}^{s-1} \sum_{d \in H_{2}(X, \mathbb{Z})} e^{\int_{d} \tau_{2}}\left\langle\gamma_{1}, \gamma_{2}, \widetilde{T}_{a}\right\rangle_{0,3, d} T^{a}
$$

whenever this sum is convergent. 
Remark 2.7. - 1. Using the notation of correlators (see (A.19)) one can define, for any $\tau$ in $H^{2 *}(X)$, a big twisted quantum product :

$$
\gamma_{1} \bullet_{\tau}^{\mathrm{tw}} \gamma_{2}:=\sum_{a=0}^{s-1}\left\langle\left\langle\gamma_{1}, \gamma_{2}, \widetilde{T}_{a}\right\rangle\right\rangle_{0} T^{a} .
$$

As usual, we have $: \bullet_{\tau_{2}}^{\mathrm{tw}}:=\left.\bullet_{\tau}^{\mathrm{tw}}\right|_{\tau=\tau_{2}}$. We will not use of big twisted quantum products.

2. One can also define the small twisted quantum product without choosing a basis by :

$$
\gamma_{1} \bullet_{\tau_{2}}^{\mathrm{tw}} \gamma_{2}:=\sum_{d \in H_{2}(X, \mathbb{Z})} e^{\int_{d} \tau_{2}} \mathrm{e}_{3 *}\left(\mathrm{e}_{1}^{*} \gamma_{1} \cup \mathrm{e}_{2}^{*} \gamma_{2} \cup c_{\mathrm{top}}\left(\mathcal{E}_{0,3, d}(3)\right) \cap\left[X_{0,3, d}\right]^{\mathrm{vir}}\right)
$$

2.1.b. Parameters. - The quantum product written in Definition 2.6 depends on the parameter $\tau_{2}$ in $H^{2}(X)$. The Picard group $\operatorname{Pic}(X)$ acts on $H^{2}(X)$ in the following way : for $\mathcal{L}$ in $\operatorname{Pic}(X), \mathcal{L} \cdot \tau_{2}=\tau_{2}+2 \sqrt{-1} \pi c_{1}(\mathcal{L})$. The number $e^{\int_{d} \tau_{2}}$ being invariant by this action, the quantum product is naturally defined over $H^{2}(X) / \operatorname{Pic}(X)=H^{2}(X) / 2 \sqrt{-1} \pi H^{2}(X, \mathbb{Z})$.

Let us extend the locus of the parameter. Denote by $\mathrm{NE}(X)_{\mathbb{Z}} \subset H_{2}(X, \mathbb{Z})$ the Mori cone of $X$, generated as a semi-group by numerical classes of irreducible curves in $X$.

Notation 2.9. - The semigroup algebras of $\mathrm{NE}(X)_{\mathbb{Z}}$ and $H_{2}(X, \mathbb{Z})$ will be respectively denoted by $\Lambda$ and $\Pi$ :

$$
\Lambda=\mathbb{C}\left[\mathrm{NE}(X)_{\mathbb{Z}}\right]=\mathbb{C}\left[Q^{d}, d \in \mathrm{NE}(X)_{\mathbb{Z}}\right], \quad \Pi=\mathbb{C}\left[H_{2}(X, \mathbb{Z})\right]=\mathbb{C}\left[Q^{d}, d \in H_{2}(X, \mathbb{Z})\right]
$$

where $Q^{d}$ are indeterminates satisfying relations : $Q^{d} \cdot Q^{d^{\prime}}=Q^{d+d^{\prime}}$.

The scheme $\operatorname{Spec} \Lambda$ is an irreducible, possibly singular, affine variety of dimension $r$. Denote by $\mathbf{V}$ the set of complex points of Spec $\Lambda$. Points of $\mathbf{V}$ are characters ${ }^{(1)}$ of $\mathrm{NE}(X)_{\mathbb{Z}}$. If $q$ is such a character, denote by $q^{d}$ its evaluation on $d$ in $\operatorname{NE}(X)_{\mathbb{Z}}$. Since $X$ is projective, the Mori cone is strictly convex and there exists a unique character sending any $d$ in $H_{2}(X, \mathbb{Z}) \backslash\{0\}$ to 0 . We will denote this character by 0 and call it, as usual, the large radius limit of $X$.

The scheme Spec $\Pi$ is a torus of dimension $r=\operatorname{rk} H_{2}(X, \mathbb{Z})$. The set of complex points of Spec $\Pi$ will be denoted by $\mathbf{T}$; a point of $\mathbf{T}$ is a character of $H_{2}(X, \mathbb{Z})$ and $\mathbf{T}$ is a smooth subset of $\mathbf{V}$. We will identify $\mathbf{T}$ and $H^{2}(X) / 2 \sqrt{-1} \pi H^{2}(X, \mathbb{Z})$ via the natural surjective morphism of complex variety :

$$
\begin{aligned}
\Psi: H^{2}(X, \mathbb{C}) & \longrightarrow \mathbf{T} \\
\tau & \longmapsto q_{\tau}:=\left[d \in H_{2}(X, \mathbb{Z}) \mapsto q_{\tau}^{d}=e^{\int_{d} \tau}\right]
\end{aligned}
$$

The kernel of $\Psi$ is $2 \sqrt{-1} \pi H^{2}(X, \mathbb{Z})$. Thus, the large radius limit 0 in $\mathbf{V} \supset \mathbf{T}$ is a limit in $H^{2}(X) / 2 \sqrt{-1} \pi H^{2}(X, \mathbb{Z})$.

The small quantum product can now be defined with parameter $q$ in $\mathbf{V}$ :

Definition 2.11. - Let $q$ be in $\mathbf{V}$ and $\gamma_{1}, \gamma_{2}$ be in $H^{2 *}(X)$. The twisted small quantum product is defined by

$$
\gamma_{1} \bullet_{q}^{\mathrm{tw}} \gamma_{2}:=\sum_{a=0}^{s-1} \sum_{d \in H_{2}(X, \mathbb{Z})} q^{d}\left\langle\gamma_{1}, \gamma_{2}, \widetilde{T}_{a}\right\rangle_{0,3, d} T^{a}
$$

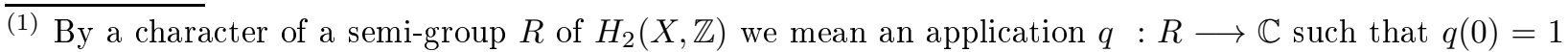
and $q\left(d+d^{\prime}\right)=q(d) \cdot q\left(d^{\prime}\right)$ for any $d, d^{\prime}$ in $R$. If $R$ is a group the image of $q$ is in $\mathbb{C}^{*}$. If $q$ is such a character, we will write $q^{d}:=q(d)$. A character $q$ of a semi-group $R$ gives a complex point Spec $\mathbb{C} \longrightarrow \operatorname{Spec} \mathbb{C}[R]$ which will also be denoted by $q$; this correspondence is a bijection. Notice that, if $d$ is in $R, Q^{d}$ is a function on Spec $_{\mathbb{C}} \mathbb{C}[R]$ and we have : $Q^{d}(q)=q^{d}$.
} 
whenever this sum is convergent.

Definition 2.11 and Definition 2.6 are compatible : For any $\tau_{2}$ in $H^{2}(X), \Psi\left(\tau_{2}\right)$ is in $\mathbf{T}$ and

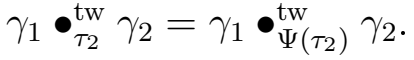

Assumption 2.12. - We will assume that there exists an open subset $\bar{V}$ of $\mathrm{V}$ containing the large radius limit 0 such that :

$$
\forall q \in \bar{V}, \forall \gamma_{1}, \gamma_{2} \in H^{2 *}(X), \gamma_{1} \bullet{ }_{q}^{\mathrm{tw}} \gamma_{2} \text { is convergent. }
$$

This assumption is easily shown to be true when the line bundle $\left(\omega_{X} \otimes \mathcal{L}_{1} \otimes \cdots \otimes \mathcal{L}_{k}\right)^{\vee}$ is ample, that is when the complete intersection variety $Z$ defined by $\mathcal{E}$ is Fano. In other cases, such as Calabi-Yau subvarieties of toric varieties considered below, one may use [Iri07] to check this assumption.

Notation 2.13. - We denote by $V$ the complex nonsingular variety $V:=\bar{V} \cap \mathbf{T}$.

Thus, $V$ is a smooth locus in $\mathbf{V}$ where the quantum product is convergent. We have :

$$
\begin{aligned}
& \text { large radius limit }=0 \in \bar{V} \text { (convergent product) } \subset \quad \mathbf{V}=\operatorname{Spec}_{\mathbb{C}} \Lambda \\
& \cup \quad \cup \\
& 0 \notin V \text { (convergent product) } \subset \mathbf{T}=\operatorname{Spec}_{\mathbb{C}} \Pi \stackrel{\sim}{\longrightarrow}\left(\mathbb{C}^{*}\right)^{r}
\end{aligned}
$$

As a convention, we will denote neighborhood of 0 in $\mathbf{V}$ by an overlined capital letter, and its intersection with $\mathbf{T}$ by the same capital letter without overlining ( $\mathbf{V}$ is a compactification of $\mathbf{T}$ in the neighbourhood of the large radius limit).

Let us recall some properties of the twisted quantum product :

Proposition 2.14. - For any $q$ in $\bar{V}$ the twisted quantum product $\bullet_{q}^{\mathrm{tw}}$ is associative, commutative, with unity $T_{0}:=1$.

Proof. - This is a classical proof, as soon as the twisted Gromov-Witten axioms are known. The twisted axioms are shown in Appendix A. Such proves are given by Pandharipande in [Pan98], Proposition 3, for a smooth hypersurface of $\mathbb{P}^{n}$ and by Iritani in Remark 2.2. of [Iri11], in the general case.

2.1.c. The trivial bundle with an integrable connexion. - Using basis $T_{1}, \ldots, T_{r}$ and $B_{1}, \ldots$, $B_{r}$ defined in 2.1, we have : $\Pi=\mathbb{C}\left[H_{2}(X, \mathbb{Z})\right] \stackrel{\sim}{\longrightarrow} \mathbb{C}\left[q_{1}^{ \pm}, \ldots, q_{r}^{ \pm}\right]$where $q_{a}:=Q^{B_{a}}$ (cf. footnote $1)$. Thus if $d=\sum_{a=1}^{r} d_{a} B_{a}$ we get $Q^{d}=\prod_{a=1}^{r} q_{a}^{d_{a}}$ in $\Pi$. Viewing the $q_{a}$ 's as coordinates of $\mathbf{T}$, we get : $q^{d}=\prod_{a=1}^{r} q_{a}^{d_{a}}$ for any $q \in \mathbf{T}$.

For $a$ in $\{1, \ldots, r\}$, we put :

$$
\delta_{a}:=q_{a} \partial_{q_{a}} \quad \delta_{z}:=z \partial_{z} .
$$

Recall that $t_{0}$ is the coordinate on $H^{0}(X)$.

Notation 2.15. - We denote by $F$ the trivial holomorphic vector bundle of fiber $H^{2 *}(X)$ over $H^{0}(X) \times V \times \mathbb{C}$ :

$$
F:=\left[H^{2 *}(X) \times\left(H^{0}(X) \times V \times \mathbb{C}\right) \rightarrow\left(H^{0}(X) \times V \times \mathbb{C}\right)\right]
$$

together with the following meromorphic connection :

$$
\nabla_{\partial_{t_{0}}}:=\partial_{t_{0}}+\frac{1}{z} \mathbf{1} \bullet_{q}^{\mathrm{tw}}, \quad \nabla_{\delta a}:=\delta_{a}+\frac{1}{z} T_{a} \bullet_{q}^{\mathrm{tw}}, \quad \nabla_{\delta_{z}}:=\delta_{z}-\frac{1}{z} \mathfrak{E} \bullet{ }_{q}^{\mathrm{tw}}+\mu
$$

where $\mu$ is the diagonal morphism defined by $\mu\left(T_{a}\right):=\frac{1}{2}\left(\operatorname{deg}\left(T_{a}\right)-\left(\operatorname{dim}_{\mathbb{C}} X-\operatorname{rk} \mathcal{E}\right)\right) T_{a}$ and $\mathfrak{E}\left(t_{0}, q, z\right):=t_{0} \mathbf{1}+c_{1}\left(\mathcal{T}_{X} \otimes \mathcal{E}^{\vee}\right)$. This global section $\mathfrak{E}$ of $F$ corresponds to the Euler field. Notice that the twisted product $\bullet_{q}^{\text {tw }}$ does not depend on $t_{0}$ because of the twisted fundamental class Axiom ( $c f$. Proposition A.4). 
In the untwisted case, it is known that $\nabla$ is a flat connection and its flat sections can be described explicitly. Let us give the equivalent property in the twisted case. We define the multi-valuated cohomological meromorphic function $L^{\mathrm{tw}}\left(t_{0}, q, z\right)$ :

$$
H^{2 *}(X) \longrightarrow H^{2 *}(X)
$$

$$
\gamma \longmapsto L^{\mathrm{tw}}\left(t_{0}, q, z\right) \gamma=e^{-t_{0} / z}\left(q^{-T / z} \gamma-\sum_{a=0}^{s-1} \sum_{\substack{H_{2}(X, Z) \\ d \neq 0}} q^{d}\left\langle\frac{q^{-T / z} \gamma}{z+\psi}, \widetilde{T}_{a}\right\rangle_{0,2, d} T^{a}\right)
$$

where

$$
\begin{aligned}
& \psi:=\psi_{1}=c_{1}\left(\mathfrak{N}_{1}\right) \text { is the class of } H^{2}\left(X_{0,3, d}\right) \text { given before Definition 2.5, } \\
& \frac{1}{z+\psi}:=\sum_{k \in \mathbb{N}}(-1)^{k} \psi^{k} z^{-k-1} \\
& q^{-T / z}=q^{-T_{1} / z} \cdots . q^{-T_{r} / z}:=e^{-z^{-1} \sum_{a=1}^{r} T_{a} \log \left(q_{a}\right)} \text { and }
\end{aligned}
$$

$\log \left(q_{a}\right)$ is the multi-valuated function, or any determination of the logarithm on a simply connected open subset of $V$.

For an endomorphism $u$, we denote $z^{u}:=\exp (u \log z)$. The following Proposition is the "twisted" version of Proposition 2.4 in [Iri09].

Proposition 2.17. - 1. The connection $\nabla$ is flat.

2. For $a$ in $\{1, \ldots, r\}$ and $\gamma \in H^{2 *}(X)$ we have

$$
\begin{aligned}
& \nabla_{\partial_{t_{0}}} L^{\mathrm{tw}}\left(t_{0}, q, z\right) \gamma=0, \quad \nabla_{\delta_{a}} L^{\mathrm{tw}}\left(t_{0}, q, z\right) \gamma=0 \\
& \nabla_{\delta_{z}} L^{\mathrm{tw}}\left(t_{0}, q, z\right) \gamma=L^{\mathrm{tw}}\left(t_{0}, q, z\right)\left(\mu-\frac{c_{1}\left(\mathcal{T}_{X} \otimes \mathcal{E}^{\vee}\right)}{z}\right) \gamma
\end{aligned}
$$

3. The multi-valued cohomological function $L^{\mathrm{tw}}\left(t_{0}, q, z\right) z^{-\mu} z^{c_{1}\left(\mathcal{T}_{X} \otimes \mathcal{E}^{\vee}\right)}$ is a fundamental solution of $\nabla$ above $H^{0}(X) \times V \times \mathbb{C}$.

Notice that, as a fundamental solution, $L^{\text {tw }}$ is convergent above $H^{0}(X) \times V \times \mathbb{C}$.

This kind of result is classical in the untwisted case ([CK99], [Iri09]). By lack references on twisted Gromov-Witten invariants, we write down a proof in full details in Appendix B.

2.1.d. The degenerated pairing. - Denote by $(\cdot, \cdot)$ the Poincaré duality on $H^{2 *}(X)$. As $\left\langle\gamma_{1}, \gamma_{2}, \widetilde{T}_{a}\right\rangle_{0,3, d}$ is not symmetric in the three arguments we do not have the Frobenius relation, that is :

$$
\left(\gamma_{1} \bullet{ }_{q}^{\mathrm{tw}} \gamma_{2}, \gamma_{3}\right) \neq\left(\gamma_{1}, \gamma_{2} \bullet \bullet_{q}^{\mathrm{tw}} \gamma_{3}\right)
$$

Nevertheless we can define a symmetric bilinear form :

Definition 2.18. - The twisted pairing on $H^{2 *}(X)$ is defined by :

$$
\forall \gamma_{1}, \gamma_{2} \in H^{2 *}(X),\left(\gamma_{1}, \gamma_{2}\right)^{\mathrm{tw}}:=\int_{X} \gamma_{1} \cup \gamma_{2} \cup c_{\text {top }}(\mathcal{E})
$$

Proposition 2.19. - 1. The bilinear form $(\cdot, \cdot)^{\mathrm{tw}}$ is degenerated with kernel ker $m_{c_{t o p}}$ where $m_{\text {ctop }}$ is define as :

$$
\begin{aligned}
m_{c_{\text {top }}}: H^{2 *}(X) & \longrightarrow H^{2 *}(X) \\
\alpha & \longmapsto c_{\mathrm{top}}(\mathcal{E}) \cup \alpha
\end{aligned}
$$


2. For $\gamma_{1}, \gamma_{2}, \gamma_{3}$ in $H^{*}(X)$, we have the Frobenius relation:

$$
\left(\gamma_{1} \bullet \bullet_{q}^{\mathrm{tw}} \gamma_{2}, \gamma_{3}\right)^{\mathrm{tw}}=\left(\gamma_{1}, \gamma_{2} \bullet \bullet_{q}^{\mathrm{tw}} \gamma_{3}\right)^{\mathrm{tw}}
$$

Proof. - The first claim is obvious.

By Definition 2.18 and Remark 2.8, it is enough to prove the following equality for any $d \in H_{2}(X, \mathbb{Z}):$

$$
\begin{aligned}
& \int_{X} \mathrm{e}_{3 *}\left(\mathrm{e}_{1}^{*} \gamma_{1} \cup \mathrm{e}_{2}^{*} \gamma_{2} \cup c_{\mathrm{top}}\left(\mathcal{E}_{0,3, d}(3)\right) \cap\left[X_{0,3, d}\right]^{\mathrm{vir}}\right) \cup \gamma_{3} \cup c_{\mathrm{top}}(\mathcal{E}) \\
= & \int_{X} \mathrm{e}_{3 *}\left(\mathrm{e}_{1}^{*} \gamma_{2} \cup \mathrm{e}_{2}^{*} \gamma_{3} \cup c_{\mathrm{top}}\left(\mathcal{E}_{0,3, d}(3)\right) \cap\left[X_{0,3, d}\right]^{\mathrm{vir}}\right) \cup \gamma_{1} \cup c_{\mathrm{top}}(\mathcal{E}) .
\end{aligned}
$$

The exact sequence :

$$
0 \longrightarrow \mathcal{E}_{0,3, d}(3) \longrightarrow \mathcal{E}_{0,3, d} \longrightarrow \mathrm{e}_{3}^{*} \mathcal{E} \longrightarrow 0
$$

gives $c_{\text {top }}\left(\mathcal{E}_{0,3, d}(3)\right) \cdot c_{\text {top }}\left(\mathrm{e}_{3}^{*} \mathcal{E}\right)=c_{\text {top }}\left(\mathcal{E}_{0,3, d}\right)$. By projection formula we get :

$$
\begin{aligned}
& \int_{X} \mathrm{e}_{3 *}\left(\mathrm{e}_{1}^{*} \gamma_{1} \cup \mathrm{e}_{2}^{*} \gamma_{2} \cup c_{\text {top }}\left(\mathcal{E}_{0,3, d}(3)\right) \cap\left[X_{0,3, d}\right]^{\mathrm{vir}}\right) \cup \gamma_{3} \cup c_{\text {top }}(\mathcal{E}) \\
= & \int_{\left[X_{0,3, d}\right]^{\mathrm{vir}}} \mathrm{e}_{1}^{*} \gamma_{1} \cup \mathrm{e}_{2}^{*} \gamma_{2} \cup \mathrm{e}_{3}^{*} \gamma_{3} \cup c_{\mathrm{top}}\left(\mathcal{E}_{0,3, d}\right)
\end{aligned}
$$

As the last number is invariant by permuting the class $\gamma_{i}$, we deduce the proposition.

Let $\mathcal{O}:=\mathcal{O}_{H^{0}(X) \times V \times \mathbb{C}}$ be the sheaf of holomorphic functions on $H^{0}(X) \times V \times \mathbb{C}$, and $\mathcal{F}$ be the sheaf of holomorphic sections of $F$. Let $\Gamma(\mathcal{O})$ be the ring of global sections of $\mathcal{O}$, and $\Gamma(\mathcal{F})$ be the $\Gamma(\mathcal{O})$-modules of global section of $F ; \Gamma(\mathcal{O})$ is endowed with the involution :

$$
\begin{aligned}
& \kappa: \quad \Gamma(\mathcal{O}) \longrightarrow \Gamma(\mathcal{O}) \\
& f\left(t_{0}, q, z\right) \longmapsto f^{\kappa}:=f\left(t_{0}, q,-z\right)
\end{aligned}
$$

Denote by $\Gamma(\mathcal{F})^{\kappa}$ the $\Gamma(\mathcal{O})$-module equals, as a set, to $\Gamma(\mathcal{F})$ and endowed with the following multiplication : $\forall f \in \Gamma(\mathcal{O}), s \in \Gamma(\mathcal{F}), f . s:=f^{\kappa} . s$. We define a a sesquilinear pairing

$$
S: \Gamma(\mathcal{F})^{\kappa} \otimes \Gamma(\mathcal{F}) \longrightarrow \Gamma(\mathcal{O})
$$

by fixing its value on constant sections of $F$ :

$$
\forall \gamma_{1}, \gamma_{2} \in H^{2 *}(X), S\left(\gamma_{1}, \gamma_{2}\right)=\left(\gamma_{1}, \gamma_{2}\right)^{\mathrm{tw}} .
$$

As a consequence, we get :

$$
\begin{aligned}
\forall s_{1}, s_{2} \in \Gamma(\mathcal{F}), \forall\left(t_{0}, q, z\right) \in H^{0}(X) \times V \times \mathbb{C}, \\
\quad S\left(s_{1}, s_{2}\right)\left(t_{0}, q, z\right)=\left(s_{1}\left(t_{0}, q,-z\right), s_{2}\left(t_{0}, q, z\right)\right)^{\mathrm{tw}} .
\end{aligned}
$$

Proposition 2.20. - 1. The pairing $S$ is $\nabla$-flat.

2. For any $s_{1}, s_{2}$ in $\Gamma(\mathcal{F})$,

$$
S\left(L^{\mathrm{tw}} \cdot s_{1}, L^{\mathrm{tw}} \cdot s_{2}\right)=S\left(s_{1}, s_{2}\right) .
$$

3. For any $\gamma_{1}, \gamma_{2}$ in $H^{2 *}(X)$ we have

$$
\begin{aligned}
& S\left(L^{\mathrm{tw}}\left(t_{0}, q, z\right) z^{-\mu} z^{c_{1}\left(\mathcal{T}_{X} \otimes \mathcal{E}^{\vee}\right)} \gamma_{1}, L^{\mathrm{tw}}\left(t_{0}, q, z\right) z^{-\mu} z^{c_{1}\left(\mathcal{T}_{X} \otimes \mathcal{E}^{\vee}\right)} \gamma_{2}\right) \\
= & S\left(e^{\sqrt{-1} \pi c_{1}\left(\mathcal{T}_{X} \otimes \mathcal{E}^{\vee}\right)} \gamma_{1}, e^{\sqrt{-1} \pi \mu} \gamma_{2}\right) .
\end{aligned}
$$


Proof. - 1. By the Frobenius property of Proposition 2.19.(2), for any $a \in\{1, \ldots, r\}$ and for any $s_{1}, s_{2} \in \Gamma(\mathcal{F})$, we have :

$$
\begin{aligned}
\delta_{a} S\left(s_{1}, s_{2}\right) & =S\left(\nabla_{\delta_{a}} s_{1}, s_{2}\right)+S\left(s_{1}, \nabla_{\delta_{a}} s_{2}\right) \\
\partial_{t_{0}} S\left(s_{1}, s_{2}\right) & =S\left(\nabla_{\partial_{t_{0}}} s_{1}, s_{2}\right)+S\left(s_{1}, \nabla_{\partial_{t_{0}}} s_{2}\right) .
\end{aligned}
$$

By the definition of $\mu$ and Proposition 2.19.(2), for any $s_{1}, s_{2} \in \Gamma(\mathcal{F})$ we have

$$
\delta_{z} S\left(s_{1}, s_{2}\right)=S\left(\nabla_{\delta_{z}} s_{1}, s_{2}\right)+S\left(s_{1}, \nabla_{\delta_{z}} s_{2}\right) .
$$

Hence, $S$ is $\nabla$-flat.

2. By flatness of $S$ and Proposition 2.17.(2), we deduce that

$$
\forall \gamma_{1}, \gamma_{2} \in H^{2 *}(X), \quad \delta_{a} S\left(L^{\mathrm{tw}} \gamma_{1}, L^{\mathrm{tw}} \gamma_{2}\right)=0 .
$$

So the expression $\left(L^{\mathrm{tw}}\left(t_{0}, q,-z\right) \gamma_{1}, L^{\mathrm{tw}}\left(t_{0}, q, z\right) \gamma_{2}\right)^{\mathrm{tw}}$ does not depend on $q$. By the asymptotic of $L^{\mathrm{tw}}$ at the large radius limit, we get

$$
\left(L^{\mathrm{tw}}\left(t_{0}, q,-z\right) \gamma_{1}, L^{\mathrm{tw}}\left(t_{0}, q, z\right) \gamma_{2}\right)^{\mathrm{tw}} \sim_{q=0}\left(q^{-T / z} \gamma_{1}, q^{T / z} \gamma_{2}\right)^{\mathrm{tw}}=\left(\gamma_{1}, \gamma_{2}\right)^{\mathrm{tw}} .
$$

The relation $S\left(L^{\mathrm{tw}} \cdot s_{1}, L^{\mathrm{tw}} \cdot s_{2}\right)=S\left(s_{1}, s_{2}\right)$ is also true for any $s_{1}, s_{2} \in \Gamma(\mathcal{F})$ by sesquilinearity.

3. By the previous formula and the flatness, we deduce that the left hand side does not depends on $z$. So we can put $z=1$. We deduce that the left hand side is equal to

$$
S\left(e^{-\sqrt{-1} \pi \mu} e^{\sqrt{-1} \pi c_{1}\left(\mathcal{T}_{X} \otimes \mathcal{E}^{\vee}\right)} \gamma_{1}, \gamma_{2}\right) .
$$

As $S\left(-\mu\left(\gamma_{1}\right), \gamma_{2}\right)=S\left(\gamma_{1}, \mu\left(\gamma_{2}\right)\right)$ for any $\gamma_{1}, \gamma_{2}$ in $H^{2 *}(X)$, we deduce the formula.

2.1.e. Integral structure. - In the same way than Iritani [Iri09, Definition 2.9] (see also [Iri11, footnote 8 p.20]), we define an integral structure on the vector bundle $F$ with connection $\nabla$, compatible to the pairing $S$.

Denote by $\gamma$ the Euler constant. For a vector bundle $\mathcal{N}$ on $X$ of rank $b$, we consider the invertible cohomology class

$$
\widehat{\Gamma}(\mathcal{N}):=\prod_{i=1}^{b} \Gamma\left(1+\nu_{i}\right)=\exp \left(-\gamma c_{1}(\mathcal{N})+\sum_{b \geq 2}(-1)^{b}(b-1) ! \zeta(b) \operatorname{Ch}_{b}(\mathcal{N})\right)
$$

where $\nu_{1}, \ldots, \nu_{b}$ are the Chern roots of $\mathcal{N}$ and $\mathrm{Ch}_{b}(\mathcal{N})$ is the class of degree $2 b$ of the Chern character $\operatorname{Ch}(\mathcal{N})$. Denote by $K(X)$ the Grothendieck group of vector bundles on $X$. Recall that the morphism $\mathrm{Ch}: K(X) \rightarrow H^{2 *}(X, \mathbb{Z})$ become an isomorphism after tensored by $\mathbb{C}$ (see for instance Theorem 3.25 p.283 in [Kar78]).

Definition 2.21. - For any $v$ in $K(X)$, we put

$$
\mathcal{Z}^{\mathrm{tw}}(v):=(2 \pi)^{-(n-k) / 2} L^{\mathrm{tw}}\left(t_{0}, q, z\right) z^{-\mu} z^{c_{1}\left(\mathcal{T}_{X} \otimes \mathcal{E}^{\vee}\right)} \widehat{\Gamma}\left(\mathcal{T}_{X}\right) \widehat{\Gamma}(\mathcal{E})^{-1}(2 \sqrt{-1} \pi)^{\operatorname{deg} / 2} \operatorname{Ch}(v) .
$$

We call $\mathcal{Z}^{\text {tw }}(K(X))$ the $\widehat{\Gamma}$-integral structure on $\operatorname{QDM}(X, \mathcal{E})$ and we denote it by $F_{\mathbb{Z}}$.

Remark 2.22. - Notice that $\mathcal{Z}^{\text {tw }}(v)$ is a multi-valued flat section of the bundle $(F, \nabla)$ and that $\mathcal{Z}^{\text {tw }}(K(X)) \otimes_{\mathbb{Z}} \mathbb{C}$ is the set of flat sections of $F$. We can understand the formula of $\mathcal{Z}^{\text {tw }}$ above as "the twist" by $\widehat{\Gamma}\left(\mathcal{T}_{X}\right) \widehat{\Gamma}(\mathcal{E})^{-1}$ of the natural integral structure given by $K(X)$.

$$
K(X) \stackrel{(2 \sqrt{-1} \pi)^{\operatorname{deg} / 2} \mathrm{Ch}}{\longrightarrow}(F, d) \stackrel{\widehat{\Gamma}\left(\mathcal{T}_{X}\right) \widehat{\Gamma}(\mathcal{E})^{-1}}{\longrightarrow}(F, d) \stackrel{\left.(2 \pi)^{-n / 2} L^{\mathrm{tw}}\left(t_{0}, q, z\right) z^{-\mu_{z^{c}}\left(\mathcal{T}_{X} \otimes \mathcal{E} \vee\right.}\right)}{\longrightarrow}(F, \nabla)
$$

Proposition 2.23. - For any $v_{1}, v_{2}$ in $K(X)$, we have :

$$
S\left(\mathcal{Z}^{\mathrm{tw}}\left(v_{1}\right), \mathcal{Z}^{\mathrm{tw}}\left(v_{2}\right)\right)=\int_{X} c_{\mathrm{top}}(\mathcal{E}) \operatorname{Td}\left(\mathcal{T}_{X}\right) \operatorname{Td}(\mathcal{E})^{-1} \operatorname{Ch}\left(v_{1} \otimes v_{2}^{\vee}\right)
$$


Proof. - Using Proposition 2.20.(3) and $e^{\sqrt{-1} \pi \mu}=(-1)^{\operatorname{deg} / 2}(\sqrt{-1})^{k-n}$, we deduce that

$$
\begin{aligned}
& S\left(\mathcal{Z}^{\mathrm{tw}}\left(v_{1}\right), \mathcal{Z}^{\mathrm{tw}}\left(v_{2}\right)\right) \\
= & (2 \sqrt{-1} \pi)^{k-n} \int_{X} c_{\text {top }}(\mathcal{E}) e^{\sqrt{-1} \pi c_{1}\left(\mathcal{T}_{X} \otimes \mathcal{E}^{\vee}\right)} \widehat{\Gamma}\left(\mathcal{T}_{X}\right) \widehat{\Gamma}(\mathcal{E})^{-1}(2 \sqrt{-1} \pi)^{\operatorname{deg} / 2} \operatorname{Ch}\left(v_{1}\right) \\
& \cup(-1)^{\operatorname{deg} / 2} \widehat{\Gamma}\left(\mathcal{T}_{X}\right) \widehat{\Gamma}(\mathcal{E})^{-1}(2 \sqrt{-1} \pi)^{\operatorname{deg} / 2} \operatorname{Ch}\left(v_{2}\right)
\end{aligned}
$$

We have the following facts : for any $\alpha, \beta$ in $H^{2 *}(X)$, for any $v$ in $K(X)$ and for any $\delta \in H^{2}(X)$,

$$
\begin{aligned}
\beta \cup(2 \sqrt{-1} \pi)^{\operatorname{deg} / 2} \alpha & =(2 \sqrt{-1} \pi)^{\operatorname{deg} / 2}\left(\beta /(2 \sqrt{-1} \pi)^{\operatorname{deg} \beta / 2} \cup \alpha\right) \\
\int_{X}(2 \sqrt{-1} \pi)^{\operatorname{deg} / 2} \alpha & =(2 \sqrt{-1} \pi)^{n} \int_{X} \alpha \\
(-1)^{\operatorname{deg} / 2} \Gamma(1+\delta) & =\Gamma(1-\delta)(-1)^{\operatorname{deg} / 2} \\
(-1)^{\operatorname{deg} / 2} \operatorname{Ch}(v) & =\operatorname{Ch}\left(v^{\vee}\right) .
\end{aligned}
$$

Denote by $\nu_{1}, \ldots, \nu_{n}$ the Chern root of $\mathcal{T}_{X}$ and $\epsilon_{1}, \ldots, \epsilon_{k}$ the Chern roots of $\mathcal{E}$. From the above properties, we deduce that

$$
\begin{aligned}
S\left(\mathcal{Z}^{\mathrm{tw}}\left(v_{1}\right), \mathcal{Z}^{\mathrm{tw}}\left(v_{2}\right)\right) & =\int_{X} c_{\mathrm{top}}(\mathcal{E}) e^{c_{1}\left(\mathcal{T}_{X} \otimes \mathcal{E}^{\vee}\right) / 2} \prod_{i=1}^{n} \Gamma\left(1+\frac{\nu_{i}}{2 \sqrt{-1} \pi}\right) \Gamma\left(1-\frac{\nu_{i}}{2 \sqrt{-1} \pi}\right) \\
& \cup \prod_{j=1}^{k} \Gamma\left(1+\frac{\epsilon_{j}}{2 \sqrt{-1} \pi}\right)^{-1} \Gamma\left(1-\frac{\epsilon_{j}}{2 \sqrt{-1} \pi}\right)^{-1} \operatorname{Ch}\left(v_{1} \otimes v_{2}^{\vee}\right)
\end{aligned}
$$

Using the formal identity $\Gamma(z) \Gamma(1-z)=\frac{\pi}{\sin (\pi z)}$, we deduce that

$$
\Gamma(1-z) \Gamma(1+z)=\frac{z e^{-z / 2}}{1-e^{-z}} .
$$

This implies the formula.

Recall from Definition 2.21 that we denote $F_{\mathbb{Z}}$ the integral structure $\mathcal{Z}^{\text {tw }}(K(X))$.

Definition 2.24. - The twisted quantum $\mathcal{D}$-module denoted by $\operatorname{QDM}(X, \mathcal{E})$ is the quadruple $\left(F, \nabla, S, F_{\mathbb{Z}}\right)$.

2.2. Reduced quantum $D$-module. - In this subsection we define the reduced quantum $\mathcal{D}$-module, denoted by $\overline{\mathrm{QDM}}(X, \mathcal{E})$, which is a quadruple $\left(\bar{F}, \bar{\nabla}, \bar{S}, \bar{F}_{\mathbb{Z}}\right)$. The pairing $\bar{S}$ is non-degenerated.

Recall that $m_{c_{\text {top }}}$ is the endomorphism

$$
\begin{aligned}
m_{c_{\text {top }}}: H^{2 *}(X) & \longrightarrow H^{2 *}(X) \\
\alpha & \longmapsto c_{\text {top }}(\mathcal{E}) \cup \alpha .
\end{aligned}
$$

Put $\overline{H^{2 *}(X)}:=H^{2 *}(X) / \operatorname{ker} m_{c t o p}$ and call it the reduced cohomology ring of $(X, \mathcal{E})$. Since $m_{c_{t o p}}$ is a graded morphism, the vector space $\overline{H^{2 *}(X)}$ is naturally graded. For $\gamma \in H^{2 *}(X)$, we denote by $\bar{\gamma}$ its class in $\overline{H^{2 *}(X)}$.

Denote by $\bar{F}$ the trivial bundle $\overline{H^{2 *}(X)} \times H^{0}(X) \times V \times \mathbb{C} \rightarrow H^{0}(X) \times V \times \mathbb{C}$. On $\bar{F}$, we will define a connection $\bar{\nabla}$ and a non-degenerated paring $\bar{S}$. They will be induced by those on $F$.

For any $\gamma_{1}, \gamma_{2} \in H^{2 *}(X)$, define the reduced pairing $(\cdot, \cdot)^{\text {red }}$ which is a bilinear form on $\overline{H^{2 *}(X)}$ by

$$
\left(\bar{\gamma}_{1}, \bar{\gamma}_{2}\right)^{\mathrm{red}}:=\left(\gamma_{1}, \gamma_{2}\right)^{\mathrm{tw}}
$$


By Proposition 2.19, ker $m_{c_{t o p}}$ is the kernel of the twisted pairing. It follows that the reduced pairing is a well defined and non degenerated bilinear form.

We define the pairing $\bar{S}$ as we did for $S$ but changing $(\cdot, \cdot)^{\text {tw }}$ by $(\cdot, \cdot)^{\text {red }}$ (cf. before Proposition 2.20). From (2.25), for any $s_{1}, s_{2} \in \Gamma\left(H^{0}(X) \times V \times \mathbb{C}, \mathcal{F}\right)$, we deduce that

$$
\bar{S}\left(\bar{s}_{1}, \bar{s}_{2}\right)=S\left(s_{1}, s_{2}\right)
$$

Let $\left(\phi_{0}, \ldots, \phi_{s^{\prime}-1}\right)$ be a homogeneous basis of $\overline{H^{2 *}(X)}$ and denote $\left(\phi^{0}, \ldots, \phi^{s^{\prime}-1}\right)$ its dual basis with respect to $(\cdot, \cdot)^{\text {red }}$.

Definition 2.2\%. - Let $\gamma_{1}, \ldots, \gamma_{n}$ be classes in $H^{2 *}(X)$.

1. Let $d$ be in $H_{2}(X, \mathbb{Z})$. The reduced Gromov-Witten invariant is

$$
\left\langle\bar{\gamma}_{1}, \ldots, \bar{\gamma}_{n}\right\rangle_{0, \ell, d}^{\mathrm{red}}:=\left\langle\gamma_{1}, \ldots, \widehat{c_{\mathrm{top}}(\mathcal{E})} \gamma_{n}\right\rangle_{0, \ell, d}
$$

2. The reduced quantum product is

$$
\bar{\gamma}_{1} \bullet_{q}^{\text {red }} \bar{\gamma}_{2}:=\sum_{a=0}^{s-1} \sum_{d \in H_{2}(X, \mathbb{Z})} q^{d}\left\langle\bar{\gamma}_{1}, \bar{\gamma}_{2}, \phi_{a}\right\rangle_{0,3, d}^{\text {red }} \phi^{a}
$$

Remark 2.28. - By the twisted $S_{n}$-symmetric axiom ( $c f$. Proposition A.2), the reduced Gromov-Witten invariants are well defined on the class in $\overline{H^{2 *}(X)}$. Notice that the reduced Gromov-Witten invariant are $S_{n}$ symmetric. The convergence domain of $\bullet_{q}^{\text {red }}$ contains $V$. We will restrict ourselves to $V$.

Proposition 2.29. - For any $\gamma_{1}, \gamma_{2}$ in $H^{2 *}(X)$, we have

$$
\overline{\gamma_{1} \bullet{ }_{q}^{\mathrm{tw}} \gamma_{2}}=\bar{\gamma}_{1} \bullet \underset{q}{\mathrm{red}} \bar{\gamma}_{2}
$$

Proof. - Using Formula (2.8) for the twisted quantum product we get :

$$
\overline{\gamma_{1} \bullet \stackrel{q}{q}^{\mathrm{tw}} \gamma_{2}}=\sum_{d \in H_{2}(X, \mathbb{Z})} q^{d} \overline{\mathrm{e}_{3 *} \alpha}
$$

where we put $\alpha:=\left(\mathrm{e}_{1}^{*} \gamma_{1} \cup \mathrm{e}_{2}^{*} \gamma_{2} \cup c_{\text {top }}\left(\mathcal{E}_{0,3, d}(3)\right) \cap\left[X_{0,3, d}\right]^{\text {vir }}\right)$. Denote by $\widehat{\phi}_{a} \in H^{2 *}(X)$ a lift of $\phi_{a}$. By Definition (2.25), we have

$$
\overline{\mathrm{e}_{3 *} \alpha}=\sum_{a=0}^{s^{\prime}-1}\left(\overline{\mathrm{e}_{3 *} \alpha}, \phi_{a}\right)^{\mathrm{red}} \phi^{a}=\sum_{a=0}^{s^{\prime}-1}\left(\mathrm{e}_{3 *} \alpha, \widehat{\phi}_{a}\right)^{\mathrm{tw}} \phi^{a}
$$

Using projection formula, the proposition follows from

$$
\left\langle\bar{\gamma}_{1}, \bar{\gamma}_{2}, \phi_{a}\right\rangle_{0,3, d}^{\mathrm{red}}=\left\langle\gamma_{1}, \gamma_{2}, c_{\mathrm{top}}(\mathcal{E}) \widehat{\phi}_{a}\right\rangle_{0,3, d}=\left(\mathrm{e}_{3 *} \alpha, \widehat{\phi}_{a}\right)^{\mathrm{tw}}
$$

Define the following connexion on the bundle $\bar{F}$ :

$$
\begin{aligned}
& \bar{\nabla}_{\partial_{t_{0}}}:=\partial_{t_{0}}+\frac{1}{z} \overline{\mathbf{1}} \bullet_{q}^{\mathrm{red}}, \quad \forall a \in\{1, \ldots, r\}, \quad \bar{\nabla}_{\delta a}:=\delta_{a}+\frac{1}{z} \bar{T}_{a} \bullet_{q}^{\mathrm{red}} \\
& \bar{\nabla}_{\delta_{z}}:=\delta_{z}-\frac{1}{z} \overline{\mathfrak{E}} \bullet_{q}^{\mathrm{red}}+\bar{\mu}
\end{aligned}
$$

where $\bar{\mu}$ is the diagonal morphism defined by $\bar{\mu}\left(\phi_{a}\right):=\frac{1}{2}\left(\operatorname{deg}\left(\phi_{a}\right)-\left(\operatorname{dim}_{\mathbb{C}} X-\mathrm{rk} \mathcal{E}\right)\right) \phi_{a}$ and $\overline{\mathfrak{E}}:=t_{0} \overline{\mathbf{1}}+\overline{c_{1}\left(\mathcal{T}_{X} \otimes \mathcal{E}^{\vee}\right)}$.

Corollary 2.30. - For any $\gamma \in H^{2 *}(X)$, we have :

$$
\overline{\nabla \gamma}=\bar{\nabla} \bar{\gamma}
$$


Proof. - This follows from Proposition 2.29 and from $\overline{\mu\left(T_{a}\right)}=\bar{\mu}\left(\overline{T_{a}}\right)$.

Lemma 2.31. - For any $\left(t_{0}, q, z\right)$ in $H^{0}(X) \times V \times \mathbb{C}$, we have :

$$
L^{\mathrm{tw}}\left(t_{0}, q, z\right)\left(\operatorname{ker} m_{c t o p}\right)=\operatorname{ker} m_{c_{t o p}} \text {. }
$$

Proof. - Let $\gamma$ be in ker $m_{\text {ctop }}$ and $\alpha \in H^{2 *}(X)$. Since $L^{\mathrm{tw}}\left(t_{0}, q, z\right)$ is an automorphism of $H^{2 *}(X)$ and ker $m_{c t o p}$ is the kernel of the twisted pairing $(\cdot, \cdot)^{\text {tw }}$ we find, using Proposition 2.20 :

$$
\begin{aligned}
\left(\alpha, L^{\mathrm{tw}}\left(t_{0}, q, z\right) \gamma\right)^{\mathrm{tw}} & =\left(L^{\mathrm{tw}}\left(t_{0}, q,-z\right) \cdot\left(L^{\mathrm{tw}}\left(t_{0}, q,-z\right)\right)^{-1} \cdot \alpha, L^{\mathrm{tw}}\left(t_{0}, q, z\right) \gamma\right)^{\mathrm{tw}} \\
& =\left(L^{\mathrm{tw}}\left(t_{0}, q,-z\right)^{-1} \alpha, \gamma\right)^{\mathrm{tw}}=0 .
\end{aligned}
$$

Then $L^{\mathrm{tw}}\left(t_{0}, q, z\right) \gamma$ belongs to ker $m_{c t o p}$.

This lemma permit us to define a reduced $L$ function : for any $\left(t_{0}, q, z\right) \in V \times \mathbb{C}$ put

$$
\begin{aligned}
\bar{L}\left(t_{0}, q, z\right): \overline{H^{2 *}(X)} & \longrightarrow \overline{H^{2 *}(X)} \\
\bar{\gamma} & \longmapsto \bar{L}\left(t_{0}, q, z\right) \bar{\gamma}=\overline{L^{\mathrm{tw}}\left(t_{0}, q, z\right) \gamma}
\end{aligned}
$$

In the same spirit of $\S 2.1 . e$, we also get an induced integral structure on $\overline{\operatorname{QDM}}(X, \mathcal{E})$. Denote by

$$
\overline{K(X)}:=K(X) /\left\{v \mid \operatorname{Ch}(v) \in \operatorname{ker} m_{c_{t o p}}\right\} .
$$

The Chern character Ch $: K(X) \rightarrow H^{2 *}(X)$ induces a reduced Chern character $\overline{\mathrm{Ch}}: \overline{K(X)} \rightarrow$ $\overline{H^{2 *}(X)}$ which become an isomorphism after tensored by $\mathbb{C}$. For any $\bar{v} \in \overline{K(X)}$, we put

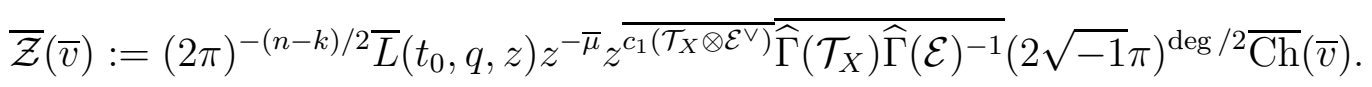

In the same spirit of Definition 2.21, the reduced $\widehat{\Gamma}$-integral structure on $\overline{\operatorname{QDM}}(X, \mathcal{E})$ is given by $\overline{\mathcal{Z}}(\overline{K(X)})$ and we denote it by $\bar{F}_{\mathbb{Z}}$.

Corollary 2.33. - The triple $(\bar{F}, \bar{\nabla}, \bar{S})$ satisfies the following properties.

1. The connection $\bar{\nabla}$ is flat and $\bar{S}$ is non-degenerated and $\bar{\nabla}$-flat.

2. A fundamental solution of $\bar{\nabla}$ is given by $\bar{L}\left(t_{0}, q, z\right) z^{-\bar{\mu}} z^{\overline{c_{1}\left(\mathcal{T}_{X} \otimes \mathcal{E}^{\vee}\right)}}$.

3. For any $\bar{s}_{1}, \bar{s}_{2} \in \Gamma(\overline{\mathcal{F}})$, we have

$$
\bar{S}\left(\bar{L}(q, z) \bar{s}_{1}, \bar{L}(q, z) \bar{s}_{2}\right)=\bar{S}\left(\bar{s}_{1}, \bar{s}_{2}\right)
$$

4. For any $v$ in $K(X)$, we have $\overline{\mathcal{Z}}(\bar{v})=\overline{\mathcal{Z}^{\mathrm{tw}}(v)}$.

5. For any $v_{1}, v_{2}$ in $K(X)$, we have

$$
\bar{S}\left(\overline{\mathcal{Z}}\left(\bar{v}_{1}\right), \overline{\mathcal{Z}}\left(\bar{v}_{2}\right)\right)=\int_{X} c_{\text {top }}(\mathcal{E}) \operatorname{Td}\left(\mathcal{T}_{X}\right) \operatorname{Td}(\mathcal{E})^{-1} \operatorname{Ch}\left(v_{1} \otimes v_{2}^{\vee}\right) .
$$

Proof. - (1) Proposition 2.17 and Corollary 2.30 implies the flatness for $\bar{\nabla}$. The flatness of $\bar{S}$ follows from Proposition 2.20 and Equality (2.26).

(2) This statement follows easily from Corollary 2.30 and Proposition 2.17.

(3) The equality follows from Proposition 2.20 and Equality (2.26).

(4) This follows from the statement (2).

(5) The equality follows from the previous equality, Equation (2.26) and Proposition 2.23.

Definition 2.34. - The reduced quantum $\mathcal{D}$-module associated to the pair $(X, \mathcal{E})$ is the quadruple $\left(\bar{F}, \bar{\nabla}, \bar{S}, \bar{F}_{\mathbb{Z}}\right)$ denoted by $\overline{\mathrm{QDM}}(X, \mathcal{E})$.

Remark 2.35. - $\quad$ 1. The set $\overline{\mathcal{Z}}(\overline{K(X)}) \otimes_{\mathbb{Z}} \mathbb{C}$ is the set of flat sections of $\overline{\operatorname{QDM}}(X, \mathcal{E})$, 
2. The reduced $\widehat{\Gamma}$-integral structure on $\overline{\mathrm{QDM}}(X, \mathcal{E})$ defined above is the one induced by the $\widehat{\Gamma}$-integral structure on $\operatorname{QDM}(X, \mathcal{E})$ defined in Definition 2.21 i.e., we have $\overline{\mathcal{Z}}(\overline{K(X)})=$ $\overline{\mathcal{Z}^{\mathrm{tw}}(K(X))}$.

\subsection{Geometric interpretation of the Reduced Quantum $\mathcal{D}$-module for complete intersection subvarieties. -}

Assumption 2.36. - In this section, we assume that $\operatorname{dim}_{\mathbb{C}} X \geq k+3$ and that the line bundles $\mathcal{L}_{1}, \ldots, \mathcal{L}_{k}$ are ample. This makes it possible to use Hyperplane and Hard Lefschetz's Theorems.

Notation 2.37. - Fix a generic section of $\mathcal{E}$, and denote by $Z$ the projective subvariety defined by this section. By Bertini's theorem, $Z$ is a smooth complete intersection subvariety of $X$. Denote by $\iota: Z \hookrightarrow X$ the corresponding closed embedding.

By Lefschetz's theorem we have

$$
H^{2 *}(Z)=\operatorname{Im} \iota^{*} \oplus \operatorname{ker} \iota_{*}
$$

and $\operatorname{ker} \iota_{*} \subset H^{\operatorname{dim}_{\mathbb{C}} Z}(Z)$. We put $H_{\mathrm{amb}}^{2 *}(Z):=\operatorname{Im} \iota^{*}$, this is the part of the cohomology of $Z$ coming from the ambient space $X$. We have the following commutative diagram

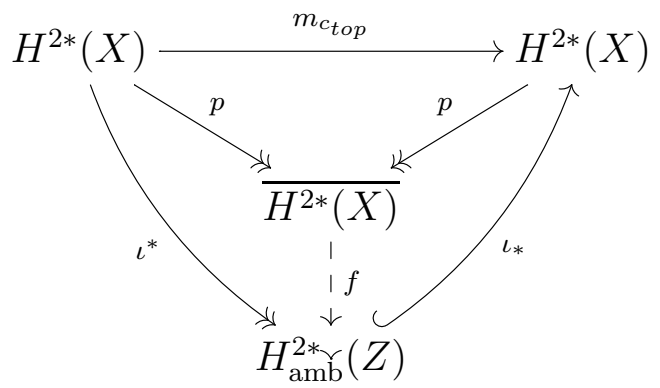

where $p$ is the natural projection and $f: \bar{\gamma} \mapsto \iota^{*} \gamma$. By the decomposition (2.38), the morphism $f$ is an isomorphism. In particular we have an isomorphism $H^{2}(X) \simeq H^{2}(Z)$ and $H^{0}(X) \simeq H^{0}(Z)$.

Remark 2.40. - It should be possible to improve Assumption 2.36, at least for toric varieties. For example, if $X$ is a toric projective variety of dimension at least $3, k=1$ and $\mathcal{L}_{1}$ is a nef (not necessary ample) line bundle on $X$, then Theorem 5.1 of [Mav00] ensures that $Z$ is a smooth connected hypersurface satisfying : $H^{2 *}(Z)=\operatorname{Im} \iota^{*} \oplus \operatorname{ker} \iota_{*}$.

Proposition 2.41. - Using Notation 2.37, and under Assumption 2.36, for any $\gamma_{1}, \gamma_{2} \in$ $H^{2 *}(X), \tau_{2} \in H^{2}(X)$,

$$
\iota^{*}\left(\gamma_{1} \bullet_{\tau_{2}}^{\mathrm{tw}} \gamma_{2}\right)=\iota^{*}\left(\gamma_{1}\right) \bullet_{\iota^{*}\left(\tau_{2}\right)}^{Z} \iota^{*}\left(\gamma_{2}\right)
$$

where $\bullet^{Z}$ is the quantum product on $Z$.

Proof. - The proof is given in Proposition 4 of [Pan98], for a smooth hypersurface of $\mathbb{P}^{n}$. The general case is treated by Iritani ([Iri11], Corollary 2.3.) using functoriality of virtual classes (cf. [KKP03]).

Recall that we identify $H^{0}(Z)$ with $H^{0}(X)$. The classical quantum $\mathcal{D}$-module associated to $Z$, denoted by $\operatorname{QDM}(Z)$, is the triple $\left(F^{Z}, \nabla^{Z}, S^{Z}\right)$ where

1. $F^{Z}$ is the trivial bundle $H^{2 *}(Z) \times H^{0}(X) \times V_{Z} \times \mathbb{C} \rightarrow H^{0}(X) \times V_{Z} \times \mathbb{C}$ where $V_{Z}$ is the subset of $H^{2}(Z) / \operatorname{Pic}(Z)$ where the quantum product on $Z$ is convergent ${ }^{(2)}$,

$\overline{{ }^{(2)} \text { We use the }}$ same parameter $q$ because of the isomorphism $\iota^{*}: H^{2}(X) \simeq H^{2}(Z)$ 
2. the connection $\nabla^{Z}$ is defined via the same formula than $\nabla$ with the quantum product of $Z$ and $\mathfrak{E}:=c_{1}\left(\mathcal{T}_{Z}\right)+t_{0} \mathbf{1}$ and

$$
\mu^{Z}\left(\psi_{a}\right)=\frac{1}{2}\left(\operatorname{deg}\left(\psi_{a}\right)-\operatorname{dim}_{\mathbb{C}} Z\right) \psi_{a} .
$$

where $\left(\psi_{a}\right)$ is a basis of $H^{2 *}(Z)$.

3. The non-degenerated pairing $S^{Z}$ is defined in the same way of $S$ but with the Poincaré duality of $H^{2 *}(Z)$.

Moreover, on $\operatorname{QDM}(Z)$ Iritani defined the $\widehat{\Gamma}$-integral structure (see Definition 2.9 [Iri09]) via $\mathcal{Z}^{Z}(K(Z))$ where for any $w$ in $K(Z)$, he puts

$$
\mathcal{Z}^{Z}(w)=(2 \pi)^{-(n-k) / 2} L^{Z}\left(t_{0}, q, z\right) z^{-\mu^{Z}} z^{c_{1}\left(\mathcal{T}_{Z}\right)} \widehat{\Gamma}\left(\mathcal{T}_{Z}\right)(2 \sqrt{-1} \pi)^{\operatorname{deg} / 2} \operatorname{Ch}(w) .
$$

In Proposition 2.10 of [Iri09], he proves that $\mathcal{Z}^{Z}(w) \otimes_{\mathbb{Z}} \mathbb{C}$ is the set of flat sections of $\operatorname{QDM}(Z)$.

We consider the trivial sub-bundle of $F^{Z}$ whose fibers are $H_{\mathrm{amb}}^{2 *}(Z)$. This sub-bundle is stable by $\nabla^{Z}$ and the pairing is still non-degenerated on it. We denote $\operatorname{QDM}_{\mathrm{amb}}(Z)$ this sub-quantum $\mathcal{D}$-module. By Proposition 2.41, the base space of this bundle $F^{Z}$ could be restricted to $H^{0}(X) \times V \times \mathbb{C}$. We put $K_{\mathrm{amb}}(Z):=\iota^{*} K(X)$. We have that $K_{\mathrm{amb}}(Z) \otimes_{\mathbb{Z}} \mathbb{C}$ is isomorphic to $H_{\mathrm{amb}}^{2 *}(Z)$ via the Chern character. So $\mathcal{Z}^{Z}\left(K_{\mathrm{amb}}(Z)\right) \otimes_{\mathbb{Z}} \mathbb{C}$ is the set of flat sections of $\mathrm{QDM}_{\mathrm{amb}}(Z)$ that is $\mathcal{Z}^{Z}\left(K_{\mathrm{amb}}(Z)\right)$ define a $\widehat{\Gamma}$-integral structure on $\mathrm{QDM}_{\mathrm{amb}}(Z)$.

The integral structure put on $\operatorname{QDM}(X, \mathcal{E})$ in $\S 2.1$.e is compatible with the one defined by Iritani, that is we have the following theorem.

Theorem 2.42. - Using Notation 2.37, and under Assumption 2.36. The reduced quantum $\mathcal{D}$-module $\overline{\mathrm{QDM}}(X, \mathcal{E})$ is isomorphic to the sub-quantum $\mathcal{D}$-module $\mathrm{QDM}_{\mathrm{amb}}(Z)$ of $\mathrm{QDM}(Z)$.

Proof. - First, we get an isomorphism of bundles. We still denote it $f$. From Proposition 2.29 and Proposition 2.41, we have :

$$
f\left(\bar{\gamma}_{1} \bullet_{q}^{\mathrm{red}} \bar{\gamma}_{2}\right)=f\left(\overline{\gamma_{1} \bullet_{q}^{\mathrm{tw}} \gamma_{2}}\right)=\iota^{*}\left(\gamma_{1} \bullet_{q}^{\mathrm{tw}} \gamma_{2}\right)=\left(\iota^{*} \gamma_{1}\right) \bullet_{q}^{Z}\left(\iota^{*} \gamma_{2}\right)=f\left(\bar{\gamma}_{1}\right) \bullet_{q}^{Z} f\left(\bar{\gamma}_{2}\right) .
$$

The adjunction formula gives : $c_{1}\left(\mathcal{T}_{Z}\right)=\iota^{*} c_{1}\left(\mathcal{T}_{X} \otimes \mathcal{E}^{\vee}\right)$. Since the dimension of $Z$ is the dimension of $X$ minus the rank of $\mathcal{E}$, we deduce that $\mu^{Z}(f(\bar{\gamma}))=f(\bar{\mu}(\bar{\gamma}))$. It follows that the isomorphism of bundle $f$ satisfies :

$$
\nabla^{Z} f(\bar{\gamma})=f(\bar{\nabla} \bar{\gamma}) \text { and } \bar{S}\left(\bar{\gamma}_{1}, \bar{\gamma}_{2}\right)=S^{Z}\left(f\left(\bar{\gamma}_{1}\right), f\left(\bar{\gamma}_{2}\right)\right)
$$

Let show that for any $\bar{\gamma}$ be in $\overline{H^{2 *}(X)}$

$$
f\left(\bar{L}\left(t_{0} q, z\right) z^{-\bar{\mu}} z^{\overline{c_{1}\left(\mathcal{T}_{X} \otimes \mathcal{E}^{\vee}\right)}} \bar{\gamma}\right)=L^{Z}\left(t_{0}, q, z\right) z^{-\mu^{Z}} z^{c_{1}\left(\mathcal{T}_{Z}\right)} f(\bar{\gamma})
$$

By equation (2.43), both side are fundamental solutions of $\mathrm{QDM}_{\mathrm{amb}}(Z)$, so they differ by the conjugation of a constant matrix. At the large radius limit, they are both equivalent to

$$
f\left(e^{t_{0} / z} z^{-\bar{\mu}} z^{\overline{c_{1}\left(\mathcal{T}_{X} \otimes \mathcal{E}^{\vee}\right)}} \bar{\gamma}\right)=e^{t_{0} / z} z^{-\mu^{Z}} z^{c_{1}\left(\mathcal{T}_{Z}\right)} f(\bar{\gamma}) .
$$

This implies that the constant matrix is the identity that is we have Equality (2.44).

Let show the compatibility between the integral structures that is $f(\overline{\mathcal{Z}}(\bar{v}))=\mathcal{Z}^{Z}\left(\iota^{*} v\right)$ for $\bar{v}$ in $\overline{K(X)}$. We use Equality $(2.44)$ with $\bar{\gamma}:=\widehat{\widehat{\Gamma}\left(\mathcal{T}_{X}\right) \widehat{\Gamma}(\mathcal{E})^{-1}(-1)^{\operatorname{deg} / 2} \operatorname{Ch}(v)}$. As we have

$$
f(\bar{\gamma})=\iota^{*}\left(\widehat{\Gamma}\left(\mathcal{T}_{X}\right) \widehat{\Gamma}(\mathcal{E})^{-1}\right)(-1)^{\operatorname{deg} / 2} \iota^{*} \operatorname{Ch}(v)
$$

As we are in complete intersection, the normal bundle $N_{Z \mid X}=\iota^{*} \mathcal{E}$. So we have the following exact sequence

$$
0 \longrightarrow \mathcal{T}_{Z} \longrightarrow \iota^{*} \mathcal{T}_{X} \longrightarrow \iota^{*} \mathcal{E} \longrightarrow 0
$$


This implies that the Chern roots if $\iota^{*} \mathcal{T}_{X}$ are the Chern roots of $\mathcal{T}_{Z}$ and the Chern roots of $\iota^{*} \mathcal{E}$. We deduce that $\widehat{\Gamma}\left(\iota^{*} \mathcal{T}_{X}\right)=\widehat{\Gamma}\left(\iota^{*} \mathcal{E}\right) \widehat{\Gamma}\left(\mathcal{T}_{Z}\right)$. As the class $\widehat{\Gamma}(v)$ is compatible with pull-back, we deduce that $f(\overline{\mathcal{Z}}(\bar{v}))=\mathcal{Z}^{Z}\left(\iota^{*} v\right)$.

Denote by $(\cdot, \cdot)_{K(Z)}$ the Mukai pairing that is $\left(w_{1}, w_{2}\right)_{K(Z)}:=\chi\left(w_{2}^{\vee} \otimes w_{1}\right)$. The following proposition show a relation between the Mukai pairing in $K(Z)$ and the three pairings $S(\cdot, \cdot), \bar{S}(\cdot, \cdot), S^{Z}(\cdot, \cdot)$ on respective flat sections.

Proposition 2.46. - For any $v_{1}, v_{2}$ in $K(X)$, we have

$$
\begin{aligned}
\left(\iota^{*} v_{1}, \iota^{*} v_{2}\right)_{K(Z)} & =S^{Z}\left(\mathcal{Z}^{Z}\left(\iota^{*} v_{1}\right), \mathcal{Z}^{Z}\left(\iota^{*} v_{2}\right)\right) \\
& =\bar{S}\left(\overline{\mathcal{Z}}\left(v_{1}\right), \overline{\mathcal{Z}}\left(v_{2}\right)\right) \\
& =S\left(\mathcal{Z}^{\mathrm{tw}}\left(v_{1}\right), \mathcal{Z}^{\mathrm{tw}}\left(v_{2}\right)\right) .
\end{aligned}
$$

Proof. - The first equality follows from Proposition 2.10 of [Iri09]. From Proposition 2.23 and Corollary 2.33, it is enough to prove that

$$
\left(\iota^{*} v_{1}, \iota^{*} v_{2}\right)_{K(Z)}=\int_{X} c_{\text {top }}(\mathcal{E}) \operatorname{Td}\left(\mathcal{T}_{X}\right) \operatorname{Td}(\mathcal{E})^{-1} \operatorname{Ch}\left(v_{1} \otimes v_{2}^{\vee}\right)
$$

From the exact sequence (2.45), we deduce that $\operatorname{Td}\left(\mathcal{T}_{Z}\right)=\iota^{*}\left(\operatorname{Td}(\mathcal{E})^{-1} \operatorname{Td}\left(\mathcal{T}_{X}\right)\right)$. By RiemannRoch and the projection formula, we have

$$
\begin{aligned}
\left(\iota^{*} v_{1}, \iota^{*} v_{2}\right)_{K(Z)} & =\chi\left(\iota^{*} v_{1} \otimes \iota^{*} v_{2}^{\vee}\right) \\
& =\int_{Z} \operatorname{Td}\left(\mathcal{T}_{Z}\right) \iota^{*} \operatorname{Ch}\left(v_{1} \otimes v_{2}^{\vee}\right) \\
& =\int_{X} \iota_{*} \iota^{*}\left(\operatorname{Td}(\mathcal{E})^{-1} \operatorname{Td}\left(\mathcal{T}_{X}\right) \operatorname{Ch}\left(v_{1} \otimes v_{2}^{\vee}\right)\right)
\end{aligned}
$$

The last equality is exactly $(2.47)$.

\section{Batyrev rings for toric varieties with a splitted vector bundle}

From now on, $X$ is a toric smooth projective variety endowed with $k$ globally generated line bundles $\mathcal{L}_{1}, \ldots, \mathcal{L}_{k}$.

In [Bat93], Batyrev constructs a ring based on the combinatorial data of a smooth toric, projective variety. In the Fano case, it is the quantum cohomology ring of this variety. As shown in [Iri11], it is also the restriction at $z=0$ of the quantum $\mathcal{D}$-module.

In this section, we define the Batyrev ring associated to the data $\left(X, \mathcal{L}_{1}, \ldots, \mathcal{L}_{k}\right)$. It is constructed as the classical Batyrev ring of a quasi-projective toric variety, namely the total space of $\mathcal{E}^{\vee}$, denoted by $Y$. This construction could be generalized to any quasi-projective, smooth toric variety defined by a convex fan.

More precisely, we prove the three following results that will be used in the rest of the paper.

1. The total space $Y$ of $\mathcal{E}^{\vee}$ is a quasi-projective smooth toric variety defined by a convex fan $\Delta$. The Batyrev ring $B$ of a $Y$ is a quotient of the ring $\Lambda\left[x_{\rho}\right]:=\Lambda\left[x_{\rho}, \rho \in \Delta(1)\right]$, where $\Lambda$ is the Novikov ring previously defined, and $\Delta(1)$ is the set of rays of the fan $\Delta$. The quotient is made by the sum of two ideals, respectively denoted by QSR (Quantum Stanley-Reisner ideal) and Lin (Linear ideal). Our first result in Theorem 3.22 gives a Groebner basis of QSR in terms of primitive collections of the fan (see Notation 3.19).

2. Moreover, assuming that the anticanonical divisor of $Y$ is nef then there exists a Zariski neighborhood $\bar{U}$ of the large radius limit in $\Lambda$ such that Spec $B \rightarrow \bar{U}$ is finite, flat of degree $\operatorname{dim} H^{2 *}(X)$ (see Theorem 3.26). 
3. To set up our last result we first define the residual Batyrev ring, denoted by $B^{\text {res }}$ (see Definition 3.39) : it is the quotient of $\Lambda\left[x_{\rho}\right]$ by the quotient ideal $\left(G: x_{\text {top }}\right)$ of $G:=\mathrm{QSR}+$ Lin by a monomial $x_{\text {top }}$ defined in terms of the $\mathcal{L}_{i}$. We show that $\operatorname{Spec} B^{\text {res }} \rightarrow \bar{U}$ is finite, flat of degree $\operatorname{dim} H^{2 *}(X)-\operatorname{dim} \operatorname{ker} m_{c_{t o p}}$ (see Proposition $3.40)$.

Subsections 3.1, 3.2 and 3.3 are preliminary results :

- some recalls of toric geometry to construct the fan of $Y$,

- the definition of the Batyrev ring,

- the definition of primitive collections and classes.

Subsection 3.4 is devoted to the proof of Theorem 3.22 on Groebner basis. In the last two subsections, we prove Theorem 3.26 on Batyrev ring and then Proposition 3.40 on residual Batyrev ring.

3.1. Notations for toric varieties. - This section is mainly based on [Ful93] and [Mus].

Denote by $N$ a $n$-dimensional lattice and by $M$ its dual lattice. Consider a fan $\Sigma$ of $N_{\mathbb{R}}=N \otimes \mathbb{R}$ and denote by $\Sigma(l)$ the set of $l$-dimensional cones of $\sigma$. The set of rays of $\Sigma$ is $\Sigma(1)=\left\{\theta_{1}, \ldots, \theta_{m}\right\}$, and for any $\theta \in \Sigma(1)$ we denote by $w_{\theta}$ the generator of $\theta \cap N$.

The $n$-dimensional toric variety defined by $\Sigma$ is denoted by $X$. For any cone $\sigma \in \Sigma$ we denote by $U(\sigma)$ the affine variety :

$$
\operatorname{Spec} \mathbb{C}\left[\sigma^{\vee}\right]:=\operatorname{Spec} \mathbb{C}\left[\chi^{u}, u \in M, \forall x \in \sigma,\langle u, x\rangle \geq 0\right]
$$

where $\chi^{u}$ are indeterminates. To any ray $\theta \in \Sigma(1)$, there is an associated toric Weil divisors denoted by $D_{\theta}$.

We assume that :

1. $\Sigma$ is non singular i.e., for any ray $\sigma \in \Sigma$, the set $\left\{w_{\theta}, \theta \in \Sigma(1), \theta \subset \sigma\right\}$ is part of a basis of the lattice $N$. This is equivalent to $X$ being smooth.

2. $X$ is projective.

Let $\mathcal{L}_{1}, \ldots, \mathcal{L}_{k}$ be $k$ globally generated line bundle over $X$, and $\mathcal{E}:=\oplus_{i=1}^{k} \mathcal{L}_{i}$. Let $L_{1}, \ldots, L_{k}$ be $k$ toric divisors such that $\mathcal{L}_{i}=\mathcal{O}\left(L_{i}\right)$. We write :

$$
L_{i}=\sum_{\theta \in \Sigma(1)} \ell_{\theta}^{i} D_{\theta}, \quad \ell_{\theta}^{i} \in \mathbb{Z}, i=1, \ldots, k
$$

Fan of the total space of $\mathcal{E}^{\vee}$. - Consider the $n+k$ dimensional lattice $N^{\prime}:=N \oplus \mathbb{Z}^{k}$. Let $\left(\epsilon_{1}, \ldots, \epsilon_{k}\right)$ be the canonical base of $\mathbb{Z}^{k}$. Denote by :

$$
\phi: N^{\prime}=N \times \mathbb{Z}^{k} \longrightarrow N
$$

the natural projection. Define a fan $\Delta$ in $N_{\mathbb{R}}^{\prime}:=N^{\prime} \otimes \mathbb{R}$ in the following way :

- The rays of $\Delta$ are indexed by $\Sigma(1) \cup\left\{L_{1}, \ldots, L_{k}\right\}$ :

$$
\begin{cases}\text { For } \theta \in \Sigma(1), & \text { put } v_{\theta}:=\left(w_{\theta}, 0\right)+\sum_{i=1}^{k} \ell_{\theta}^{i}\left(0, \epsilon_{i}\right), \\ \text { For } i=1, \ldots, k, & \text { put } v_{L_{i}}:=\left(0, \epsilon_{i}\right) .\end{cases}
$$

Then,

$$
\Delta(1):=\left\{\rho_{\theta}:=\mathbb{R}^{+} v_{\theta}, \theta \in \Sigma(1)\right\} \cup\left\{\rho_{L_{i}}:=\mathbb{R}^{+} v_{L_{i}}, i=1, \ldots, k\right\} .
$$

- a strongly convex polyhedral cone $\sigma$ is in $\Delta$ if and only if $\phi(\sigma) \in \Sigma$.

By assumption, the line bundles $\mathcal{L}_{i}$ are globally generated and the function $\psi_{L_{i}}$ associated to each toric divisor $L_{i}$ is concave. This gives :

Fact 3.1. - As the line bundles $\mathcal{L}_{1}, \ldots, \mathcal{L}_{k}$ are globally generated, the support $|\Delta|=\cup_{\sigma \in \Delta} \sigma$ of the fan $\Delta$ in $N_{\mathbb{R}}^{\prime}$ is convex (we will say that $\Delta$ is convex). 
It will be convenient to make the distinction between rays $\rho_{\theta}$ coming from the base variety $X$, and rays $\rho_{L_{i}}$ coming from the splitted vector bundle $\mathcal{E}$.

Notation 3.2. - We put :

$$
\Delta_{(1)}^{\text {base }}=\left\{\rho_{\theta}, \theta \in \Sigma(1)\right\}, \quad \Delta_{(1)}^{\text {v.b. }}=\left\{\rho_{L_{1}}, \ldots, \rho_{L_{k}}\right\} .
$$

so that $\Delta(1)=\Delta_{(1)}^{\text {base }} \sqcup \Delta_{(1)}^{\text {v.b. }}$.

Let $Y$ be the toric variety associated to $\Delta$. As $X$ is smooth, $Y$ is also smooth. We denote by the same letter

$$
\phi: Y \longrightarrow X
$$

the scheme morphism induced by the projection $\phi: N^{\prime} \longrightarrow N$.

The next proposition gives a geometric interpretation of the toric variety $Y$ :

\section{Proposition 3.3 ([CLS11], Proposition 7.3.1 and Exercise 7.3.3)}

The toric variety $Y$ is the total space of the dual vector bundle $\mathcal{E}^{\vee} ;$ the toric morphism $\phi: Y \rightarrow X$ is the natural projection of this vector bundle.

One can easily check the following result about cohomology classes :

Proposition 3.4. - The projection $\phi: Y \longrightarrow X$ induces an isomorphism :

$$
\phi^{*}: H^{*}(X) \stackrel{\sim}{\longrightarrow} H^{*}(Y) .
$$

Moreover, if $i$ is in $\{1, \ldots, k\}$ and $D_{i}$ is the divisor of $Y$ corresponding to the ray $\rho_{L_{i}}$ (see construction 3.1), we have :

$$
\left[L_{i}\right]=\phi^{*}\left[-D_{i}\right] \text { in } H^{2}(X)
$$

Example 3.5. - Consider the fan of $\mathbb{P}^{1}$ given by $\left(N=\mathbb{Z}, w_{1}=1, w_{2}=-1\right), \mathcal{L}=\mathcal{O}(2)$ and $L=2 D_{1}$. The fan $\Delta$ is given by the rays $v_{\theta_{1}}=(1,2), v_{\theta_{2}}=(-1,0)$ an $v_{L}=(0,1)$ (cf. Figure 1).

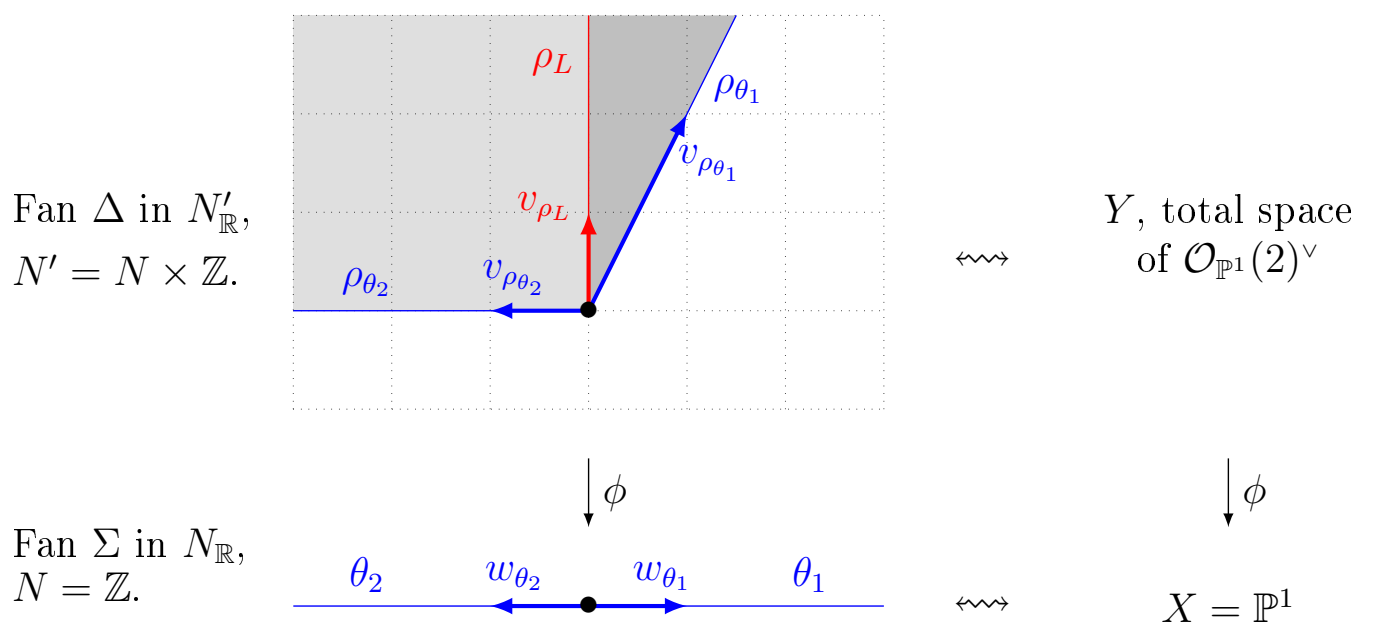

Figure 1. Fans $\Sigma$ and $\Delta$ associated to $X=\mathbb{P}^{1}, \mathcal{L}=\mathcal{O}\left(2 D_{1}\right)$ 
3.2. Batyrev ring of a quasi-projective fan.- We define and study the Batyrev ring of the fan $\Delta$ defined in section 3.1.

Remark 3.6. - Notice that all the results of this part remain true for any fan $\Gamma$ in a lattice $L$ such that :

1. $\Gamma$ is non singular i.e., defines a smooth variety.

2. The support of $\Gamma$ is convex of maximal dimension $\operatorname{dim} L$.

3. $\Gamma$ defines a quasi-projective variety.

Denote by $\mathrm{NE}(Y)_{\mathbb{Z}} \subset H_{2}(Y, \mathbb{Z})$ the (integral) Mori cone of $Y$ :

$$
\mathrm{NE}(Y)_{\mathbb{Z}}=\left\{\sum_{\text {finite sum }} n_{C}[C], n_{C} \in \mathbb{N},[C] \text { numeric class of irreducible curve }\right\} \text {. }
$$

The (integral) nef cone in $H^{2}(Y, \mathbb{Z})$ is the dual cone to $\mathrm{NE}(Y)_{\mathbb{Z}}$. It is generated by globally generated divisors.

Following [CvR08], to each toric Weil divisor $L=\sum_{\theta \in \Delta(1)} \ell_{\theta} D_{\theta}$ is associated a piecewise linear functions $\psi_{L}$ from the support $|\Delta|$ of $\Delta$ to $\mathbb{R}$ which is linear on any cone of $\Delta$, integral on $N^{\prime}$, and satisfies :

$$
\forall \theta \in \Delta(1), \psi_{L}\left(w_{\theta}\right)=-\ell_{\theta} .
$$

Denote by $\operatorname{PL}(\Delta)$ the set of piecewise linear functions from $|\Delta|$ to $\mathbb{R}$ which are linear on any cone of $\Delta$ and integral on $N^{\prime} . \operatorname{PL}(\Delta)$ is isomorphic to the set of toric divisors of $Y$, which is also isomorphic to the set $\bigoplus_{\rho \in \Delta(1)} \mathbb{Z} D_{\rho}$ (recall that $Y$ is smooth). There is an exact sequence :

$$
0 \longrightarrow M^{\prime} \longrightarrow \mathrm{PL}(\Delta)=\mathbb{Z}^{\Delta(1)} \longrightarrow H^{2}(Y, \mathbb{Z}) \longrightarrow 0 .
$$

Let $\operatorname{CPL}(\Delta) \subset \operatorname{PL}(\Delta)$ be the subset of concave functions, then the image of $\operatorname{CPL}(\Delta)$ by the map $\operatorname{PL}(\Delta) \longrightarrow H^{2}(Y, \mathbb{Q})$ is the Nef cone of $Y$.

Also recall that the ample cone of $Y$ is the interior of the nef cone. It is the image by $\mathrm{PL}(\Delta) \otimes \mathbb{R} \longrightarrow H^{2}(Y, \mathbb{R})$ of the set of strictly concave piecewise linear functions of $|\Delta|$ (cf. [Mus], Chap. 6). Since $Y$ is a quasi projective variety, the ample cone is non empty and its dimension is equal to $r=\operatorname{dim} H^{2}(Y, \mathbb{R})$.

We define the coefficient ring of $Y$ by :

$$
\Lambda:=\mathbb{C}\left[\mathrm{NE}(Y)_{\mathbb{Z}}\right]=\mathbb{C}\left[Q^{d}, d \in \mathrm{NE}(Y)_{\mathbb{Z}}\right] .
$$

Remark 3.8. - In this article, $Y$ is a fiber bundle of base $X$. As a consequence, the cohomology groups, nef cones, Mori cones of $X$ and $Y$ are isomorphic, and $\Lambda=\mathbb{C}\left[\mathrm{NE}(Y)_{\mathbb{Z}}\right]=$ $\mathbb{C}\left[\mathrm{NE}(X)_{\mathbb{Z}}\right]$ (see Proposition 3.4).

Let $d$ be a class of $H_{2}(Y, \mathbb{Q})$. We put

$$
d_{\rho}:=D_{\rho} . d=\int_{d} D_{\rho} .
$$

For any ray $\rho$ of $\Delta$. Dualizing the exact sequence 3.7 gives :

$$
0 \longrightarrow H_{2}(Y, \mathbb{Q}) \longrightarrow \mathbb{Q}^{\Delta(1)} \longrightarrow N_{\mathbb{Q}} \longrightarrow 0,
$$

Where the image of $d \in H_{2}(Y, \mathbb{Q})$ by the left arrow is $\left(d_{\rho}\right)_{\rho \in \Delta(1)} \in \mathbb{Q}^{\Delta(1)}$.

For any real number $a$, we also put $a^{+}=\max (a, 0), a^{-}=\max (-a, 0)$ so that : $a=a^{+}-a^{-}$. Finally put $d^{+}=\left(d_{\rho}^{+}\right)_{\rho \in \Delta(1)}$ and $d^{-}=\left(d_{\rho}^{-}\right)_{\rho \in \Delta(1)}$. Identifying $d \in H_{2}(Y, \mathbb{Z})$ and its image in $\mathbb{Z}^{\Delta(1)}$ (see the exact sequence 3.9), we have :

$$
d=d^{+}-d^{-} .
$$


Consider a set of indeterminate $x_{\rho}, \rho \in \Delta(1)$, corresponding to the set of rays of $\Delta$, and the single indeterminate $z$. We put :

$$
\Lambda\left[x_{\rho}\right]:=\Lambda\left[x_{\rho}, \rho \in \Delta(1)\right] .
$$

For any $d \in H_{2}(Y, \mathbb{Z})$ denote by $R_{d}$ the polynomial :

$$
R_{d}:=x^{d^{+}}-Q^{d} x^{d^{-}}=\prod_{\rho \in \Delta(1)} x_{\rho}^{d_{\rho}^{+}}-Q^{d} \prod_{\rho \in \Delta(1)} x_{\rho}^{d_{\rho}^{-}} .
$$

The quantum Stanley-Reisner ideal of $\Lambda\left[x_{\rho}\right]$ is the ideal QSR generated by the $R_{d}$ :

$$
\text { QSR }:=\left\langle R_{d}, d \in \mathrm{NE}(Y)_{\mathbb{Z}}\right\rangle
$$

The linear ideal of $\Lambda\left[x_{\rho}\right]$, is the ideal Lin generated by the following linear polynomials $Z_{u}$ 's :

$$
\operatorname{Lin}:=\left\langle Z_{u}:=\sum_{\rho \in \Delta(1)}\left\langle u, v_{\rho}\right\rangle x_{\rho}, u \in M^{\prime}\right\rangle
$$

Definition 3.12. - The Batyrev ring of $\Delta$ is the ring :

$$
B:=\Lambda\left[x_{\rho}\right] / G,
$$

where $G:=$ QSR + Lin is the sum of the quantum Stanley-Reisner and linear ideal.

Remark 3.13. - Suppose that $N^{\prime}$ is equipped with a basis $\left(e_{1}, \ldots, e_{n^{\prime}}\right)$. In that situation, we will put

$$
\forall i=1, \ldots, n^{\prime}, Z_{i}:=Z_{e_{i}^{*}} \text {, }
$$

where $\left(e_{1}^{*}, \ldots, e_{n^{\prime}}^{*}\right)$ is the dual basis of $\left(e_{1}, \ldots, e_{n^{\prime}}\right)$ in $M^{\prime}$. The linear ideal Lin is generated by $Z_{1}, \ldots, Z_{n^{\prime}}$.

3.3. Primitive collections. — Following Batyrev ([Bat93]) and Cox ([CvR08]) we define :

Definition 3.14. - A subset $\left\{\rho_{1}, \ldots, \rho_{l}\right\}$ of $\Delta(1)$ is called a primitive collection for $\Delta$ if $\left\{\rho_{1}, \ldots, \rho_{l}\right\}$ is not contained in a single cone of $\Delta$ but every proper subset is.

Let $C=\left\{\rho_{1}, \ldots, \rho_{l}\right\}$ be a primitive collection, and $v_{1}, \ldots, v_{l}$ be the generating vectors of $\rho_{1} \cap N^{\prime}, \ldots, \rho_{l} \cap N^{\prime}$. Let $\sigma$ be the minimal cone of $\Delta$ containing $v=\sum_{i=1}^{l} v_{i}$. Denote by $\rho_{1}^{\prime}, \ldots, \rho_{s}^{\prime}$ the rays of $\sigma$ and $v_{1}^{\prime}, \ldots, v_{s}^{\prime}$ the primitive vectors of the $\rho_{i}^{\prime}$. Since $\sigma$ is the minimal cone of $\Delta$ containing $v$, the vector $v$ is in the relative interior of $\sigma$ and there exists $s$ positive numbers $a_{i}$ such that : $v=a_{1} v_{1}^{\prime}+\cdots+a_{s} v_{s}^{\prime}$. Moreover, since $v$ is in $N^{\prime}$ and the $v_{j}^{\prime}$ are part of a basis of $N^{\prime}$ ( $\Delta$ is non singular), then the $a_{j}$ 's are uniquely defined in $\mathbb{N}_{>0}$.

Remark 3.15. - With the above notations : $\left\{v_{1}, \ldots, v_{l}\right\} \cap\left\{v_{1}^{\prime}, \ldots, v_{s}^{\prime}\right\}=\varnothing$. (See proposition 1.9 of [CvR08]).

Let $C=\left\{\rho_{1}, \ldots, \rho_{l}\right\}$ be a primitive collection and $v=\sum_{i=1}^{l} v_{i}=a_{1} v_{1}^{\prime}+\cdots+a_{s} v_{s}^{\prime}$ be as above. Then

$$
\sum_{i=1}^{l} v_{i}-\sum_{j=1}^{s} a_{j} v_{j}^{\prime}=0
$$

The exact sequence 3.9 shows that $H_{2}(Y, \mathbb{Z})=\operatorname{ker}\left(\mathbb{Z}^{\Delta(1)} \longrightarrow N^{\prime}\right)$, and there exists a well defined element $\left.d_{C} \in H_{2}(Y, \mathbb{Z})\right)$ such that :

$$
d_{\rho}^{C}=D_{\rho} . d^{C}=\int_{d^{C}} D_{\rho}=\left\{\begin{array}{cl}
1 & \text { if } \rho \in C \\
-a_{j} & \text { if } \rho=\mathbb{R}^{+} v_{j}^{\prime}, j \in\{1, \ldots, s\} \\
0 & \text { otherwise }
\end{array}\right.
$$


Definition 3.16. - The class of a primitive collection $C$ is the class $d^{C} \in H_{2}(Y, \mathbb{Z})$ defined as above.

Lemma 3.17. - Let $C$ be a primitive collection, then $d^{C} \in N E(Y)_{\mathbb{Z}}$.

Proof. - See [CvR08], proposition 1.9.

Also recall the following result from [CvR08] (Proposition 1.10) :

Proposition 3.18. - The Mori cone $N E(Y)_{\mathbb{Z}}$ is generated by classes of primitive collections.

A similar proposition for the Stanley-Reisner ideal will be proved in the next section.

Notation 3.19. - The set of primitive classes of $\Delta$ is :

$$
\mathcal{P}:=\left\{d^{C} \mid C \text { primitive collection of } \Delta\right\} .
$$

\subsection{Monomial order and Groebner basis.}

3.4.a. Monomial order on the variables $x_{\rho}$. - We fix, once and for all, a monomial order $\prec$ on the variables $x_{\rho}$ and a strictly concave piecewise-linear function $\varphi$ of $|\Delta|$, rational on $N^{\prime}$. Since $\Delta$ is quasi-projective, such a function exists. Denote by $O=\sum_{\rho \in \Delta(1)}-\varphi\left(v_{\rho}\right) D_{\rho}$ the ample linear $\mathbb{Q}$-divisor defined by $\varphi$.

Let $x^{a}:=\prod_{\rho \in \Delta(1)} x_{\rho}^{a_{\rho}}\left(a \in \mathbb{N}^{\Delta(1)}\right)$ be a monomial in $\Lambda\left[x_{\rho}\right]$. Put :

$$
\omega\left(x^{a}\right)=\sum_{\rho \in \Delta(1)}-a_{\rho} \varphi\left(v_{\rho}\right)
$$

and define a monomial order $\prec_{\varphi}$ as follows :

$$
x^{a} \prec_{\varphi} x^{a^{\prime}} \Longleftrightarrow\left\{\begin{array}{l}
\omega\left(x^{a}\right)<\omega\left(x^{a^{\prime}}\right) \\
\text { or } \\
\omega\left(x^{a}\right)=\omega\left(x^{a^{\prime}}\right) \text { and } x^{a} \prec x^{a^{\prime}} .
\end{array}\right.
$$

Let $P \in \Lambda\left[x_{\rho}\right]$ be a polynomial. The leading term of $P$ for $\prec_{\varphi}$ will be $\operatorname{denoted}$ by $\operatorname{Lt}(P)$. If $\operatorname{Lt}(P)=\alpha x^{a}$ with $\alpha \in \Lambda$ and $a \in \mathbb{N}^{\Delta(1)}$, then $\alpha$ is the leading coefficient of $P$, denoted by $\operatorname{Lc}(P)$ and $x^{a}$ is its leading monomial, denoted by $\operatorname{Lm}(P)$. Since $\Lambda$ is not a field, this distinction between leading terms and leading monomials is necessary.

Lemma 3.20. - Let $d$ be in the Mori cone $N E(Y)_{\mathbb{Z}}, R_{d}=x^{d^{+}}-Q^{d} x^{d^{-}}$, then $\operatorname{Lt}\left(R_{d}\right)=$ $\operatorname{Lm}\left(R_{d}\right)=x^{d^{+}}$.

Proof. - We have : $R_{d}=x^{d^{+}}-Q^{d} x^{d^{-}}$, and :

$$
\omega\left(x^{d^{+}}\right)-\omega\left(x^{d^{-}}\right)=\sum_{\rho \in \Delta(1)}-d_{\rho}^{+} \varphi\left(v_{\rho}\right)-\sum_{\rho \in \Delta(1)}-d_{\rho}^{-} \varphi\left(v_{\rho}\right)=\sum_{\rho \in \Delta(1)}-d_{\rho} \varphi\left(v_{\rho}\right)=O . d>0 .
$$

\section{4.b. Groebner basis of the quantum Stanley-Reisner ideal.-}

Definition 3.21. - Let $a$ be in $H_{2}(Y, \mathbb{Z})$ or in $\mathbb{Z}^{\Delta(1)}$. We say that $a$ is supported by a cone if the set $\left\{\rho \in \Delta(1) \mid a_{\rho} \neq 0\right\}$ is contained in a cone of $\Delta$.

We can now give a Groebner basis of QSR for the monomial order $\prec_{\varphi}$. Recall that the set of primitive classes is denoted by $\mathcal{P}$ (Notation 3.19). We have : 
Theorem 3.22. - The set $\left\{R_{d}, d \in \mathcal{P}\right\}$ is a Groebner basis with respect to the order $\prec_{\varphi}$ of the quantum Stanley-Reisner ideal QSR defined in 3.11. Moreover, the set Lt(QSR) := $\{\operatorname{Lt}(P), P \in \mathrm{QSR}\}$, is :

$$
\mathrm{Lt}(\mathrm{QSR})=\left\{\alpha x^{a} \mid \alpha \in \Lambda, \text { a is not supported by a cone. }\right\} \text {. }
$$

Remark 3.23. - (1) Being a Groebner basis over the coefficient ring $\Lambda$-which is not a field-, means that the initial terms of the polynomials $R_{d}, d \in \mathcal{P}$, generate the ideal $\langle\operatorname{Lt}(\mathrm{QSR})\rangle$ in $\Lambda\left[x_{\rho}\right]$. Notice that all the $R_{d}, d \in \mathcal{P}$ have a leading coefficient equals to 1 , so that the Groebner basis property remains true at any point of $\operatorname{Spec} \Lambda$.

(2) The ideal QSR should be seen as a toric ideal over a ring. Toric ideals over a field, are studied in [Stu96] where a similar result to Theorem 3.22 is proved.

First prove the following proposition :

Proposition 3.24. - Let $K$ be the fraction field of $\Lambda$. Let $\mathrm{QSR}^{\prime}$ be the ideal of $K\left[x_{\rho}\right]$ generated by $\left\{R_{d}, d \in N E(Y)_{\mathbb{Z}}\right\}$, then :

(i) $\mathrm{QSR}^{\prime}=\left\langle R_{d}, d \in \mathcal{P}\right\rangle=\left\langle R_{d}, d \in H_{2}(Y, \mathbb{Z})\right\rangle$ in $K\left[x_{\rho}\right]$.

(ii) The set $\left\{R_{d}, d \in \mathcal{P}\right\}$ is a Groebner basis of $\mathrm{QSR}^{\prime}$ in $K\left[x_{\rho}\right]$.

Proof. - Put $A:=\left\{R_{d}, d \in \mathcal{P}\right\}$, and apply the multivariate algorithm to $A$ (see [Eis95], algorithm 15.7). Consider the set

$$
\begin{aligned}
E:=\left\{R_{d}, d \in H_{2}(Y, \mathbb{Z}) \mid\right. & \text { any possible remainder of a } \\
& \text { multivariate division of } \left.R_{d} \text { by } A \text { is not zero }\right\} .
\end{aligned}
$$

Let us prove that $E$ is empty. Assume that it is not. Denote by $d \in H_{2}(Y, \mathbb{Z})$ a class such that $R_{d}$ is in $E$, and is a minimal element of $E$ for the order $\prec_{\varphi}$. Since $R_{d}=x^{d^{+}}-Q^{d} x^{d^{-}}$, two cases can occur :

a) $\operatorname{Lt}\left(R_{d}\right)=x^{d^{+}}$.

In this case $d^{+}$is not supported by a cone. Because if $d^{+}$is supported by a cone, then $-d \in \mathrm{NE}(Y)_{\mathbb{Z}}$ by Lemma 3.25.(2) and $\operatorname{Lt}\left(R_{-d}\right)=x^{(-d)^{+}}=x^{d^{-}}$by Lemma 3.20; this gives $\operatorname{Lt}\left(R_{d}\right)=Q^{d} x^{d^{-}}$which is a contradiction.

Then there exists a primitive collection $C$ contained in the support of $d^{+}$. Denote by $c$ the class of $C$ and put $a=d^{+}-c^{+} \in \mathbb{N}^{\Delta(1)}$. By Lemma 3.25.(3), we have :

$$
R_{d}-x^{a} R_{c}=Q^{c} x^{\min \left(d^{-}, a+c^{-}\right)} R_{d-c}=-Q^{d} x^{d^{-}}+Q^{c} x^{a+c^{-}} .
$$

Since $\operatorname{Lm}\left(R_{d}\right)=x^{d^{+}}, x^{d^{-}} \prec_{\varphi} x^{d^{+}}$and since $\operatorname{Lm}\left(R_{c}\right)=x^{c^{+}}, x^{a+c^{-}} \prec_{\varphi} x^{a+c^{+}}=x^{d^{+}}$. It follows that

$$
\begin{aligned}
\operatorname{Lm}\left(R_{d-c}\right) \preceq_{\varphi} \operatorname{Lm}\left(Q^{c} x^{\min \left(d^{-}, a+c^{-}\right)} R_{d-c}\right) & \\
& =\operatorname{Lm}\left(Q^{d} x^{d^{-}}-Q^{c} x^{a+c^{-}}\right) \in\left\{x^{d^{-}}, x^{a+c^{-}}\right\} \\
& \prec_{\varphi} x^{d^{+}}=\operatorname{Lm}\left(R_{d}\right) .
\end{aligned}
$$

Since $\operatorname{Lm}\left(R_{d}\right)$ is minimal in $E$, the polynomial $R_{d-c}$ is not in $E$, and we have

$$
R_{d}=x^{a} R_{c}+Q^{c} x^{\min \left(d^{-}, a+c^{-}\right)} R_{d-c}
$$

- We deduce that $R_{d}$ is not in $E$ which is a contradiction.

b) $\operatorname{Lt}\left(R_{d}\right)=x^{d^{-}}$.

Consider the polynomial $R_{-d}=x^{d^{-}}-Q^{-d} x^{d^{+}}$. Then we have $\operatorname{Lm}\left(R_{-d}\right)=\operatorname{Lm}\left(R_{d}\right)=x^{(-d)^{+}}$ and $R_{-d}$ is not in $E$ by $a$ ). Since $R_{d}=-Q^{d} R_{-d}$, we deduce that $R_{d}$ is not in $E$ which is a contradiction.

Thus, $E$ is empty that is, for any $d \in H_{2}(Y, \mathbb{Z})$ there exists a remainder of the division of $R_{d}$ by $A$ which is zero. We are now able to prove $(i)$ and $(i i)$ : 
(i) By Lemma 3.17 any class of a primitive collection is in the Mori cone, and we have :

$$
\langle A\rangle \subset \mathrm{QSR}^{\prime} \subset\left\langle R_{d}, d \in H_{2}(Y, \mathbb{Z})\right\rangle .
$$

Moreover, for any $d \in H_{2}(Y, \mathbb{Z})$ there exists a remainder of the division of $R_{d}$ by $A$ which is zero, hence $R_{d}$ is in the ideal $\langle A\rangle$ generated by $A$ and $\left\langle R_{d}, d \in H_{2}(Y, \mathbb{Z})\right\rangle=\langle A\rangle=\mathrm{QSR}^{\prime}$.

(ii) By $(i)$ the set $A=\left\{R_{d}, d \in \mathcal{P}\right\}$ generates QSR'. Let us apply the Buchberger's algorithm to $A$. Let $C_{1}, C_{2}$ be two primitive collections of respective classes $c_{1}, c_{2} \in \mathrm{NE}(Y)_{\mathbb{Z}}$. For $i \in\{1,2\}$ we consider the monomial :

$$
x^{a_{1}}=\frac{\operatorname{LCM}\left(\operatorname{Lm}\left(R_{c_{1}}\right), \operatorname{Lm}\left(R_{c_{2}}\right)\right)}{\operatorname{Lm}\left(R_{c_{i}}\right)}=x^{\max \left(c_{1}^{+}, c_{2}^{+}\right)-c_{i}^{+}} .
$$

By lemma 3.25.(3) we have :

$$
x^{a_{1}} R_{c_{1}}-x^{a_{2}} R_{c_{2}}=x^{\min \left(a_{1}+c_{1}^{-}, a_{2}+c_{2}^{-}\right)} Q^{c_{2}} R_{c_{1}-c_{2}} .
$$

Since the remainder of the division of $R_{c_{1}-c_{2}}$ by $A$ is zero, the set $A$ is a Groebner basis of QSR'.

Proof of Theorem 3.22. - Either in $K\left[x_{\rho}\right]$ or in $\Lambda\left[x_{\rho}\right]$ we have :

$$
\begin{aligned}
\left\langle\operatorname{Lt}\left(R_{d}\right), d \in \mathcal{P}\right\rangle & =\left\langle x^{d^{+}}, d \in \mathcal{P}\right\rangle \\
& \left.=\left\langle x^{a}, a \in \mathbb{N}^{\Delta(1)}\right| a \text { is not supported by a cone }\right\rangle .
\end{aligned}
$$

Let $P \in \mathrm{QSR} \subset \Lambda\left[x_{\rho}\right]$. As an element of $K\left[x_{\rho}\right]$, the element $P$ is in QSR'. By Proposition 3.24, $\operatorname{Lt}\left(\mathrm{QSR}^{\prime}\right)=\left\langle\operatorname{Lt}\left(R_{d}\right), d \in \mathcal{P}\right\rangle$; then $\operatorname{Lt}(P)=\alpha x^{a}, \alpha \in K$ and $a$ not supported by a cone. Since $P$ is in $\Lambda\left[x_{\rho}\right], \alpha$ is in $\Lambda$ and we are done.

Lemma 3.25. - 1. Let $d$ be in $H_{2}(Y, \mathbb{Z}), d \neq 0$, then either $d^{+}$or $d^{-}$is not supported by a cone.

2. Let $d$ be in $H_{2}(Y, \mathbb{Z})$, if $d^{-}$is supported by a cone, then $d \in N E(Y)_{\mathbb{Z}}$.

3. Let $c, d$ be in $H_{2}(Y, \mathbb{Z}), a, b$ be in $\mathbb{N}^{\Delta(1)}$. Suppose that $c^{+}+a=d^{+}+b$, then in $\mathbb{C}\left[H_{2}(Y, \mathbb{Z})\right][z]\left[x_{\rho}\right]$, we have : $x^{a} R_{c}-x^{b} R_{d}=x^{\min \left(a+c^{-}, b+d^{-}\right)} Q^{d} R_{c-d}$

Proof. - 1. We have : $\sum_{\rho \in \Delta(1)} d_{\rho}^{+} v_{\rho}=\sum_{\rho \in \Delta(1)} d_{\rho}^{-} v_{\rho}$. Let $\sigma^{+}$be the minimal cone of $\Delta(1)$ supporting $d^{+}$, and $\sigma^{-}$be the minimal cone of $\Delta$ supporting $d^{-}$.

Put $v:=\sum_{\rho \in \Delta(1)} d_{\rho}^{+} v_{\rho}=\sum_{\rho \in \Delta(1)} d_{\rho}^{-} v_{\rho}$. Then $v$ is in the interior of $\sigma^{+}$and $\sigma^{-}$. It follows that $\sigma^{+}=\sigma^{-}$and, since $\Delta$ is non singular, $d^{+}=d^{-}$and $d=0$.

2. We have to show that, for any nef toric divisor $T$, we have $T . d \geq 0$. Let $T$ be such a divisor and $\psi$ the piecewise linear concave function associated to $T$ :

$$
\begin{aligned}
T . d & =\sum_{\rho}-\psi\left(v_{\rho}\right) d_{\rho}^{+}-\sum_{\rho}-\psi\left(v_{\rho}\right) d_{\rho}^{-} \\
& =\sum_{\rho}-\psi\left(v_{\rho}\right) d_{\rho}^{+}+\psi\left(\sum_{\rho} d_{\rho}^{-} v_{\rho}\right)\left(d^{-} \text {supported by } \sigma\right) \\
& \geq-\psi\left(\sum_{\rho} d_{\rho}^{+} v_{\rho}\right)+\psi\left(\sum_{\rho} d_{\rho}^{-} v_{\rho}\right)=0\left(\psi \text { concave, and }\left(\sum_{\rho} d_{\rho}^{+} v_{\rho}=\sum_{\rho} d_{\rho}^{-} v_{\rho}\right)\right)
\end{aligned}
$$

3. Since $a+c^{+}=b+d^{+}, c^{+}-c^{-}=c$ and $d^{+}-d^{-}=d$, we have :

$$
\begin{aligned}
\min \left(a+c^{-}, b+d^{-}\right) & =\min \left(a+c^{+}-c, b+d^{-}\right)=\min \left(b+d^{+}-c, b+d^{-}\right) \\
& =\min \left(b+d^{-}+d-c, b+d^{-}\right)=b+d^{-}+\min (d-c, 0) \\
& =b+d^{-}-(c-d)^{+} .
\end{aligned}
$$


Similarly, $\min \left(a+c^{-}, b+d^{-}\right)=a+c^{-}-(c-d)^{-}$. Then we get :

$$
\begin{aligned}
x^{a} R_{c}-x^{b} R_{d} & =x^{a+c^{+}}-Q^{c} x^{a+c^{-}}-x^{b+d^{+}}+Q^{d} x^{b+d-} \\
& =x^{\min \left(a+c^{-}, b+d^{-}\right)} Q^{d} \times \\
& \quad\left(x^{b+d^{-}-\min \left(a+c^{-}, b+d^{-}\right)}-Q^{c-d} x^{a+c^{-}-\min \left(a+c^{-}, b+d^{-}\right)}\right) \\
& =x^{\min \left(a+c^{-}, b+d^{-}\right)} Q^{d}\left(x^{(c-d)^{+}}-Q^{c-d} x^{(c-d)^{-}}\right) \\
& =x^{\min \left(a+c^{-}, b+d^{-}\right)} Q^{d} R_{c-d} .
\end{aligned}
$$

\subsection{Flatness, finiteness and degree of the Batyrev ring over the coefficient ring.}

- The aim of this section is to prove the following result :

Theorem 3.26. - Let $\mathcal{L}_{1}, \ldots, \mathcal{L}_{k}$ be globally generated line bundles on $X$ such that $\left(\omega_{X} \otimes\right.$ $\left.\mathcal{L}_{1} \otimes \cdots \otimes \mathcal{L}_{k}\right)^{\vee}$ is nef. Let $B$ be the Batyrev ring of $\left(X, \mathcal{L}_{1}, \ldots, \mathcal{L}_{k}\right)$ defined above. There exists a Zariski neighbourhood $\bar{U} \subset$ Spec $\Lambda$ of the large radius limit such that the restricted scheme morphism :

is finite, flat, of degree $\operatorname{dim} H^{2 *}(X)$.

$$
\left.\operatorname{Spec}(B)\right|_{\bar{U}} \longrightarrow \bar{U}
$$

Remark 3.27. - 1. Notice that the definition of $B$ depends on the choice of the toric divisors $L_{i}$ of each $\mathcal{L}_{i}$. Differents choices of toric divisors give isomorphic rings.

2. The open subset $\bar{U}$ will be defined in paragraph 3.5.d. We call it the freeness neighbourhood of $\Delta$. It only depends on $X$, not on the vector bundle $\mathcal{E}$ and can be explicitely computed by elimination algorithm.

Recall that $Y$ is the total space of $\mathcal{E}^{\vee}$, defined by the fan $\Delta$, that $\mathrm{NE}(Y)_{\mathbb{Z}}=\operatorname{NE}(X)_{\mathbb{Z}}$ and $\left[K_{Y}\right] \in H^{2}(Y)=\left[K_{X}+L_{1}+\ldots+L_{k}\right] \in H^{2}(X)$ via the isomorphism defined in Proposition 3.4. We will rephrase Theorem 3.26 and actually prove :

If $\Delta$ is a smooth, quasi-projective, convex, fan defining a variety $Y$ and if the anticanonical divisor $-K_{Y}$ is nef, then there exists a neighbourhood $\bar{U} \subset \operatorname{Spec} \Lambda$ of the large radius limit such that the restricted scheme morphism : $\left.\operatorname{Spec}(B)\right|_{\bar{U}} \longrightarrow \bar{U}$ is finite, flat, of degree $\operatorname{dim} H^{2 *}(Y)$.

First consider the fiber of $B$ over the large radius limit.

3.5.a. Large radius limit. - Using Notations 3.34, Spec $\Lambda=\mathbf{V}$. Let $\mathbf{0}$ be the "large radius limit" point. It is defined by the maximal ideal $\mathfrak{m}=\left\langle Q^{d}, d \in \mathrm{NE}(Y)_{\mathbb{Z}}, d \neq 0\right\rangle$ in $\Lambda$. The fiber of Spec $B$ over this point is well known :

Notation 3.28. - Put :

$$
\begin{aligned}
\mathrm{SR} & =\left\langle x^{d^{+}}, d \in \mathrm{NE}(Y)_{\mathbb{Z}}\right\rangle=\left\langle x^{a}, a \in \mathbb{N}^{\Delta(1)} \text { not supported by a cone. }\right\rangle \\
\text { Lin } & =\left\langle\sum_{\rho \in \Delta(1)}\left\langle u, v_{\rho}\right\rangle x_{\rho}, u \in M^{\prime}\right\rangle
\end{aligned}
$$

The ideal SR is the Stanley-Reisner ideal of $\Delta$ (see [BH93] for example).

Proposition 3.31. - Let $\mathcal{L}_{1}, \ldots, \mathcal{L}_{k}$ be globally generated line bundles. The image of QSR (resp. Lin) in $\Lambda / \mathfrak{m}$ is SR (resp. Lin) ; there is a well defined isomorphism:

$$
\begin{array}{ccc}
B / \mathfrak{m} B=\mathbb{C}\left[x_{\rho}\right] /\langle\mathrm{SR}+\operatorname{Lin}\rangle & \stackrel{\sim}{\longrightarrow} H^{2 *}(Y, \mathbb{C})=H^{2 *}(X, \mathbb{C}) \\
x_{\rho} & \longmapsto & {\left[D_{\rho}\right]}
\end{array}
$$

where $\left[D_{\rho}\right] \in H^{2}(Y)$ is the class of the toric divisor $D_{\rho}$. 
Proof. - Since $\Delta$ is convex and quasi-projective, the proof of [Ful93] in the complete case can be adapted to our case ; then there is a well defined isomorphism :

$$
\begin{aligned}
\mathbb{Z}\left[x_{\rho}\right] /(\mathrm{SR}+\mathrm{Lin}) & \stackrel{\sim}{\longrightarrow} H^{2 *}(Y, \mathbb{Z}) \\
x_{\rho} & \longmapsto\left[D_{\rho}\right],
\end{aligned}
$$

which proves the proposition.

Notice that the fiber at $\mathfrak{m}$ does not depend on the vector bundle $\mathcal{E}$.

3.5.b. Flatness of $\Lambda \rightarrow \Lambda\left[x_{\rho}\right] /$ QSR.-

Lemma 3.32. - The morphism $\Lambda \longrightarrow \Lambda\left[x_{\rho}\right] /$ QSR is a flat morphism of relative dimension $n^{\prime}=\operatorname{dim} Y ; \Lambda$ and $\Lambda\left[x_{\rho}\right] /$ QSR both are Cohen-Macaulay rings.

Proof. - Flatness. For $P$ in $\Lambda\left[x_{\rho}\right]$, denote by $\bar{P}$ its image in $\Lambda\left[x_{\rho}\right] /$ QSR. Let $A$ be the set of monomials of $\Lambda\left[x_{\rho}\right]$ not contained in Lm(QSR). By Theorem 3.22, $A=\left\{x^{a} \in \mathbb{N}^{\Delta(1)}\right.$ $a$ is supported by a cone $\}$. As in [Eis95] (theorem 15.17) we prove that $\Lambda\left[x_{\rho}\right] /$ QSR is a free $\Lambda$-module with basis $\bar{A}=\{\bar{P}, P \in A\}$ :

Let $x^{a_{1}}, \ldots, x^{a_{l}}$ be in $A$ and $\alpha_{1}, \ldots, \alpha_{l}$ be in $\Lambda$. If $\sum_{i} \alpha_{i} \overline{x^{a_{i}}}=0$, then $\sum_{i} \alpha_{i} x^{a_{i}} \in$ QSR and $\operatorname{Lm}\left(\sum_{i} \alpha_{i} x^{a_{i}}\right) \in \operatorname{Lm}(\mathrm{QSR})$. Since all the $a_{i}^{\prime} s$ are supported by a cone, we get $\alpha_{i}=0$ for any $i=1, \ldots, l$, and $\bar{A}$ is free over $\Lambda$.

Suppose now that $\bar{A}$ does not generate $\Lambda\left[x_{\rho}\right] /$ QSR as a $\Lambda$-module. Let $x^{a}$ be the smallest monomial for $\prec_{\varphi}$ such that $\overline{x^{a}} \notin \Lambda . \bar{A}$. The $m^{\prime}$-tuple $a$ is not supported by a cone, and there exists a class $d$ of a primitive collection, and $b \in \mathbb{N}^{\Delta(1)}$ such that $x^{a}=x^{b} R_{d}+Q^{d} x^{b+d^{-}}$. We deduce that $\overline{x^{a}}=Q^{d} \overline{x^{b+d^{-}}}$. By assumption, and since $x^{b+d^{-}} \prec_{\varphi} x^{a}$, the class $\overline{x^{b+d^{-}}}$belongs to $\Lambda . \bar{A}$, hence we conclude that $\overline{x^{a}}$ is in $\Lambda . \bar{A}$ which is a contradiction.

Fiber over the large radius limit is Cohen-Macaulay of dimension $n^{\prime}$.

Let $\mathfrak{m}$ be the ideal of the point $\mathbf{0}$ in $\mathbf{V}$, as in Paragraph 3.5.a. The image of QSR in $\Lambda\left[x_{\rho}\right] / \mathfrak{m}$ is the Stanley-Reisner ring SR (see Notation 3.29). By [BH93], Theorem 5.1.4, and Corollary 5.4.6, $\mathbb{C}\left[x_{\rho}\right] / \mathrm{SR}$ is a Cohen-Macaulay ring of dimension $n^{\prime}$.

Fibers over Spec $\Lambda$ are Cohen-Macaulay of dimension $n^{\prime}$.

Let $\mathfrak{n}$ be any maximal ideal of $\Lambda$, and denote by $\overline{\text { QSR }}$ the image of QSR in $(\Lambda / \mathfrak{n})\left[x_{\rho}\right]=$ $\mathbb{C}\left[x_{\rho}\right]$. By Theorem 3.22, the set $\left\{\bar{R}_{d}, d \in \mathcal{P}\right\}$ is a Groebner basis of $\overline{\text { QSR }}$. The initial ideal of $\overline{\mathrm{QSR}}$ is $\left\langle x^{a}, a \in \mathbb{N}^{\Delta(1)}\right.$, not supported by a cone $\rangle$; this is the ideal SR studied above.

By [Eis95], there exists a flat morphism of algebras $\mathbb{C}[t] \longrightarrow C$ whose fiber $C \otimes \mathbb{C}[t] /(t)$ over 0 is $\mathbb{C}\left[x_{\rho}\right] / \mathrm{SR}$ and whose fiber $C_{p}=C \otimes \mathbb{C}[t] / p$ over any other point $p$ of Spec $\mathbb{C}[t]$ is isomorphic to $\mathbb{C}\left[x_{\rho}\right] / \overline{\mathrm{QSR}}$.

The set of $p \in \operatorname{Spec} \mathbb{C}[t]$ such that the fiber of $\operatorname{Spec}(C) \longrightarrow \operatorname{Spec} \mathbb{C}[t]$ over $p$ is CohenMacaulay is open ([Gro66], corollary 12.1.7). It is not empty since it contains 0 hence it contains a point $p \neq 0$ of $\mathbb{C}$. It follows that $\mathbb{C}\left[x_{\rho}\right] / \overline{\mathrm{QSR}}$ is Cohen-Macaulay. Moreover, by flatness of $\mathbb{C}[t] \longrightarrow C, \operatorname{dim} \mathbb{C}\left[x_{\rho}\right] / \overline{\mathrm{QSR}}=\operatorname{dim} \mathbb{C}\left[x_{\rho}\right] / \mathrm{SR}=n^{\prime}$.

As a conclusion, $\Lambda \longrightarrow \Lambda\left[x_{\rho}\right] /$ QSR is flat, of relative dimension $n^{\prime}$, and its fibers all are Cohen-Macaulay. Since Spec $\Lambda$ is a toric affine variety, it is also Cohen-Macaulay. It follows that $\Lambda\left[x_{\rho}\right] /$ QSR is Cohen-Macaulay ([BH93], Theorem 2.1.7).

We now come to the study of the Batyrev ring $B=\Lambda\left[x_{\rho}\right] /(\mathrm{QSR}+\mathrm{Lin})$. By definition, $\operatorname{Spec}(B)$ is a subscheme of $\operatorname{Spec} \Lambda\left[x_{\rho}\right]$ and there is a natural projection :

$$
\operatorname{Spec}(B) \longrightarrow \operatorname{Spec} \Lambda
$$




\section{5.c. Homogenization of $\Lambda\left[x_{\rho}\right]$.}

Put $m^{\prime}=\operatorname{Card} \Delta(1)$, consider a new variable $h$ and denote by $\mathbb{P}_{\mathrm{v}}^{m^{\prime}}$ the projective scheme Proj $\Lambda\left[x_{\rho}, h\right]$, with the grading given by $\operatorname{deg}(h)=1$ and $\operatorname{deg}\left(x_{\rho}\right)=1$. Also denote by $H$ the hyperplane at infinity defined by $h=0$, and by $\mathbb{A}_{\mathrm{v}}^{m^{\prime}}$ the affine subspace $\operatorname{Spec} \Lambda\left[x_{\rho}\right]=\mathbb{P}_{\mathbf{v}}^{m^{\prime}} \backslash H$.

The homogenization of a polynomial $P \in \Lambda\left[x_{\rho}\right]$ is $P^{h}$ :

$$
P^{h}:=h^{\operatorname{deg} P} P\left(\frac{x_{\rho}}{h}\right) \in \Lambda\left[x_{\rho}, h\right] .
$$

The linear polynomials $Z_{u}\left(u \in M^{\prime}\right)$ being homogeneous, we have $Z_{u}^{h}=Z_{u}$. As for the homogenization of $R_{d}$, notice that for any $d \in H_{2}(Y, \mathbb{Z})$, we have $\operatorname{deg}\left(x^{d^{+}}\right)-\operatorname{deg}\left(x^{d^{-}}\right)=$ $\sum_{\rho} D_{\rho} . d=-K_{Y} . d$. We get :

Remark 3.33. - If $-K_{Y}$ is nef, then for any $d$ in $\mathrm{NE}(Y)_{\mathbb{Z}}$,

$$
R_{d}^{h}=x^{d^{+}}-Q^{d} h^{-K_{Y} \cdot d} x^{d^{-}} .
$$

Let $\Gamma$ be the closed subscheme of $\mathbb{P}_{\mathbf{v}}^{m^{\prime}}$ defined by the homogeneous polynomials $R_{d}^{h}$ for $d \in \mathrm{NE}(Y)_{\mathbb{Z}}$. Let $\chi$ be the closed subscheme of $\Gamma \subset \mathbb{P}_{\mathbf{v}}^{m^{\prime}}$ defined by the polynomials $R_{d}^{h}$ for $d \in \operatorname{NE}(Y)_{\mathbb{Z}}$ and $Z_{u}$ for $u \in M^{\prime}$. We have :

$$
\chi \subset \Gamma \text { and } \operatorname{Spec} B=\chi \cap \mathbb{A}_{\mathbf{v}}^{m^{\prime}} .
$$

The closed subscheme $\chi \cap H$ is defined in Proj $\Lambda\left[x_{\rho}\right]$ by the homogeneous polynomials: $R_{d}^{\infty}$ and $Z_{u}$ where :

$$
\begin{aligned}
\forall d \in \mathrm{NE}(Y)_{\mathbb{Z}} & R_{d}^{\infty}=\left.R_{d}^{h}\right|_{h=0} \in \Lambda\left[x_{\rho}\right] \\
\forall u \in M^{\prime} & Z_{u}=\sum_{\rho \in \Delta(1)}\left\langle u, v_{\rho}\right\rangle x_{\rho} .
\end{aligned}
$$

Let $\pi$ be the natural morphism :

$$
\pi: \mathbb{P}_{\mathbf{V}}^{m^{\prime}} \longrightarrow \mathbf{V}
$$

The image $\pi(\chi \cap H)$ is a closed subset of $\mathbf{V}$.

Notation 3.34. - The closed subset of $\mathrm{V}$

$$
\bar{U}:=\mathbf{V} \backslash \pi(\chi \cap H)
$$

is called the freeness neighborhoods of the large radius limit. Its intersection $U:=\bar{U} \cap \mathbf{T}$ with the torus is also called freeness neighbourhood. This terminology is justified by Theorem 3.26 and Proposition 3.40.

Remark 3.35. - $\quad$ 1. Using primitive collections and elimination algorithms, we can give an explicit description of the algebraic open subset $\bar{U}$. Let $\left(e_{1}, \ldots, e_{n^{\prime}}\right)$ be a basis of $N^{\prime}$ as in Remark 3.13. The closed subscheme $\chi \cap H$ is defined by the finite set of polynomials : $Z_{i}=\sum_{\rho \in \Delta(1)}\left\langle e_{i}^{*}, v_{\rho}\right\rangle x_{\rho}$ and, for $d$ in the set $\mathcal{P}$ of primitive classes,

$$
R_{d}^{\infty}= \begin{cases}x^{d^{+}} & \text {if } \sum_{\rho} d_{\rho}>0 \\ x^{d^{+}}-Q^{d} x^{d^{-}} & \text {if } \sum_{\rho} d_{\rho}=0\end{cases}
$$

The ideal in $\Lambda$ of the closed subset $\pi(\chi \cap H)$ can be obtained by elimination of the variables $x_{\rho}$.

2. The homogenization of an ideal $I$ of $\Lambda\left[h, x_{\rho}\right]$ is : $I^{h}=\left\langle P^{h}, P \in I\right\rangle$. Recall that, if $I$ is generated by $P_{1}, \ldots, P_{l}$, we do not have in general $: I^{h}=\left\langle P_{1}^{h}, \ldots, P_{l}^{h}\right\rangle$. In our situation, if $\chi^{\prime}$ is the closed subscheme of $\mathbb{P}_{\mathbf{v}}^{m^{\prime}}$ defined by the homogenized ideal $G^{h}$, we only get $\chi^{\prime} \subset \chi$.

Lemma 3.36. - The large radius limit is in $\bar{U}$. 
Proof. - Using notations above, let $\mathbf{0} \in \mathbf{V}$ be the large radius limit, and $\chi_{\mathbf{0}}$ be the fiber of $\chi \longrightarrow \mathbf{V}$ over it. The intersection $\chi_{\mathbf{0}} \cap H$ is defined by the homogeneous ideal $:\langle h\rangle+\mathrm{SR}+\mathrm{Lin}$ in $\mathbb{P}_{\mathbb{C}}^{m^{\prime}}$. And we have :

$$
\mathbb{C}\left[x_{\rho}, h\right] /(\langle h\rangle+\mathrm{SR}+\mathrm{Lin}) \stackrel{\sim}{\longrightarrow} \mathbb{C}\left[x_{\rho}\right] /(\mathrm{SR}+\mathrm{Lin}),
$$

this last ring being isomorphic to $H^{2 *}(Y, \mathbb{C})$ by Proposition 3.31. It follows that the zero locus in $\mathbb{C}^{m^{\prime}}$ of the ideal $\mathrm{SR}+\operatorname{Lin} \subset \mathbb{C}\left[x_{\rho}\right]$ is defined by $x_{\rho}=0$ for any $\rho \in \Delta(1)$. Then, the reduced ideal of $\langle h\rangle+\mathrm{SR}+$ Lin is the irrelevant ideal of the graded ring $\mathbb{C}\left[h, x_{\rho}\right]$ and $\chi_{\mathbf{0}} \cap H$ is empty.

Remark 3.3\%. - (Fano subvariety) If $-K_{Y}$ is ample, that is, in our case, if the complete intersection subvariety defined by a generic section of $\mathcal{E}$ is Fano, then the freeness neighborhood $\bar{U}$ is equal to the whole set $\mathbf{V}$. Actually, since $-K_{Y} \cdot d>0$ for any $d \in \mathrm{NE}(Y)_{\mathbb{Z}}$, the projectivized polynomials $R_{d}^{\infty}$ are equals to $\left\langle x^{d^{+}}, d \in \mathrm{NE}(Y)_{\mathbb{Z}}\right\rangle$. Each fiber of $\chi \longrightarrow \mathbf{V}$ is isomorphic to $\chi_{\mathbf{0}}$ and $\chi \cap H=\varnothing$.

3.5.d. Proof of Theorem 3.26. - Consider the following diagram :

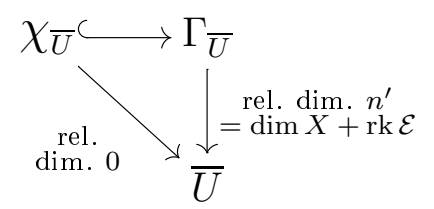

where $\Gamma_{\bar{U}}$ is the restriction of $\Gamma=\operatorname{Proj} \Lambda\left[h, x_{\rho}\right] /\left\langle R_{d}^{h}\right\rangle$ to $\bar{U}$. By Lemma 3.32. $\mathbb{A}_{\bar{U}}^{m^{\prime}} \cap \Gamma_{\bar{U}} \longrightarrow$ $\bar{U}$ is a flat morphism of relative dimension $n^{\prime}$ between Cohen-Macaulay schemes.

Above $\bar{U}$ and $\chi$ are contained in the affine part of $\Gamma$ (away from the hyperplane $H$ ) and has relative dimension zero since the fibers do not meet the hyperplane $H$. Thus, $\chi_{\bar{U}} \rightarrow \bar{U}$ is a finite and proper morphism.

Let $\left(e_{1}, \ldots, e_{n^{\prime}}\right)$ be a basis of $N^{\prime}$. We use of notations of Remark 3.13. Let $p$ be a point of $\bar{U}$ and denote by $\bar{Z}_{i}$ the image of $Z_{i}$ in the quotient of $\Lambda\left[x_{\rho}\right]$ by the maximal ideal defining $p$. In the Cohen-Macaulay fiber $\mathbb{A}_{p}^{m^{\prime}} \cap \Gamma_{p}$ over $p$, the scheme $\chi_{p}$ has codimension $n^{\prime}$ and is defined by a sequence of length $n^{\prime}$ (namely $\left(\bar{Z}_{1}, \ldots, \bar{Z}_{n^{\prime}}\right)$ ). By [BH93], theorem 2.1.2, $\left(\bar{Z}_{1}, \ldots, \bar{Z}_{n^{\prime}}\right)$ is a regular sequence.

By the corollary to the theorem 22.5 of [Mat86], since $\mathbb{A}_{\bar{U}}^{m^{\prime}} \cap \Gamma_{\bar{U}} \rightarrow \bar{U}$ is flat, and $\left(\bar{Z}_{1}, \ldots, \bar{Z}_{n^{\prime}}\right)$ are regular sequences over any point of $\bar{U}$, then $\chi_{\bar{U}} \longrightarrow \bar{U}$ is flat.

The degree of this finite morphism can be computed as the length of the fiber $\chi_{\mathbf{0}}$ over the large radius limit. By proposition 3.31 it is equal to $\operatorname{dim} H^{2 *}(Y)$.

Remark 3.38. - If $Y$ is Fano, one can also proof by induction on the degree that $B$ is a free $\Lambda$-module. A basis of $B$ is given by a free subset of the set of monomials $\left\{x^{a}, a \in\right.$ $\mathbb{N}^{\Delta(1)}, a$ is supported by a cone, $\left.a_{\rho} \in\{0,1\}\right\}$ which generates $B$.

3.6. Flatness, finiteness and degree of the residual Batyrev ring over $\bar{U}$. - As we have $H^{2}(X) \simeq H^{2}(Y)$ and, via this isomorphism, we have : $\left[L_{i}\right]=\left[-D_{\rho_{i}}\right]$ for $\rho_{i} \in \Delta^{\text {v.b. }}(1)$ (cf. Proposition 3.4).

We put :

$$
\begin{array}{lll}
c_{i}:=\left[L_{i}\right]=\left[-D_{\rho_{i}}\right] \in H^{2}(X), & c_{\mathrm{top}}(\mathcal{E}) & :=\prod_{i=1}^{k} c_{i} \in H^{2 k}(X) \\
x_{i}:=x_{\rho_{i}} \text { which is a variable in } \Lambda\left[x_{\rho}\right], & x_{\mathrm{top}} & :=(-1)^{k} \prod_{i=1}^{k} x_{i} \in \Lambda\left[x_{\rho}\right]
\end{array}
$$

We also define the quotient ideal :

$$
\left(G: x_{\mathrm{top}}\right):=\left\{P \in \Lambda\left[x_{\rho}\right], x_{\mathrm{top}} \cdot P \in \mathrm{QSR}+\mathrm{Lin}=G\right\} .
$$


Finally recall that we denoted by $m_{c_{t o p}}: H^{2 *}(Y) \longrightarrow H^{2 *}(Y)$ the morphism of multiplication by $c_{\text {top }}(\mathcal{E})$.

Definition 3.39. - The residual Batyrev ring of $\left(\Sigma, L_{1}, \ldots, L_{k}\right)$ with respect to $\mathcal{E}$ is the $\Lambda$-algebra :

$$
B^{\text {Res }}=\Lambda\left[x_{\rho}\right] /\left(G: x_{\mathrm{top}}\right) .
$$

Proposition 3.40. - Let $\mathcal{L}_{1}, \ldots, \mathcal{L}_{k}$ be ample line bundle on $X$ such that $\left(\omega_{X} \otimes \mathcal{L}_{1} \otimes \cdots \otimes\right.$ $\left.\mathcal{L}_{k}\right)^{\vee}$ is nef. Denote by $\bar{U} \subset \mathbf{V}$ the freeness neighborhood defined in 3.34. Then the restricted morphism $\left.\operatorname{Spec}\left(B^{\text {Res }}\right)\right|_{\bar{U}} \longrightarrow \bar{U}$ is finite, flat of degree $\operatorname{dim}_{\mathbb{C}} H^{2 *}(X)-\operatorname{dim} \operatorname{ker} m_{c_{t o p}}$.

Proof. - Denote by $\bar{x}_{\text {top }}$ the image of $x_{\text {top }}$ in $B=\Lambda\left[x_{\rho}\right] /(\mathrm{QSR}+\mathrm{Lin})$, and by $m_{c_{\text {top }}}: B \longrightarrow$ $B$ the morphism of multiplication by $\bar{x}_{\text {top }}$ in $B$. This multiplication induces an isomorphism :

$$
B^{\text {Res }}=\Lambda\left[x_{\rho}\right] /\left(G: x_{\text {top }}\right) \stackrel{\sim}{\longrightarrow} \bar{x}_{\text {top }} B=\operatorname{Im}\left(m_{c_{\text {top }}}\right) .
$$

(well defined and injective by definition of the quotient ideal $\left(G: x_{\mathrm{top}}\right)$ ). Moreover, there is an exact sequence :

$$
0 \longrightarrow \bar{x}_{\mathrm{top}} B \longrightarrow B \longrightarrow B / \bar{x}_{\mathrm{top}} B \longrightarrow 0
$$

By definition $B / \bar{x}_{\text {top }} B$ is isomorphic to $\Lambda\left[x_{\rho}\right] /\left(\mathrm{QSR}+\operatorname{Lin}+\left\langle x_{\mathrm{top}}\right\rangle\right)$.

Let $d$ be a class of $\mathrm{NE}(Y)_{\mathbb{Z}}$. For any $\rho=\rho_{L_{i}} \in \Delta_{(1)}^{\text {v.b. }}$, since $\left[-D_{\rho}\right]=\left[L_{i}\right]$ is ample, $d_{\rho}=D_{\rho} . d<0$ and we have :

$$
R_{d}=x^{d^{+}}-Q^{d} x_{\mathrm{top}} x^{d^{-}-\epsilon}
$$

where $\epsilon=\left(\epsilon_{\rho}\right)_{\rho \in \Delta(1)}, \epsilon_{\rho}=1$ if $\rho \in \Delta_{(1)}^{\text {v.b. }}, \epsilon_{\rho}=0$ otherwise.

As a consequence, in $\Lambda\left[x_{\rho}\right] /\left(\mathrm{QSR}+\operatorname{Lin}+\left\langle x_{\mathrm{top}}\right\rangle\right), \bar{R}_{d}=x^{d^{+}}$and we can write :

$$
\begin{aligned}
\Lambda\left[x_{\rho}\right] /\left(\mathrm{QSR}+\operatorname{Lin}+\left\langle x_{\mathrm{top}}\right\rangle\right) & \stackrel{\sim}{\longrightarrow} \Lambda\left[x_{\rho}\right] /\left(\left\langle x^{d^{+}}, d \in \mathrm{NE}(Y)_{\mathbb{Z}}\right\rangle+\left\langle Z_{u}, u \in M^{\prime}\right\rangle+\left\langle x_{\mathrm{top}}\right\rangle\right) \\
& \stackrel{\sim}{\longrightarrow} \Lambda \otimes\left(\mathbb{C}[z]\left[x_{\rho}\right] /\left(\left\langle x^{d^{+}}, d \in \mathrm{NE}(Y)_{\mathbb{Z}}\right\rangle+\left\langle Z_{u}, u \in M^{\prime}\right\rangle+\left\langle x_{\mathrm{top}}\right\rangle\right)\right)
\end{aligned}
$$

Using Proposition 3.31, we get :

$$
\mathbb{C}[z]\left[x_{\rho}\right] /\left(\left\langle x^{d^{+}}, d \in \mathrm{NE}(Y)_{\mathbb{Z}}\right\rangle+\left\langle Z_{u}, u \in M^{\prime}\right\rangle+\left\langle x_{\mathrm{top}}\right\rangle\right) \stackrel{\sim}{\longrightarrow} H^{2 *}(X, \mathbb{C}) /\left\langle\prod_{i=1}^{k} c_{1}\left(\mathcal{L}_{i}\right)\right\rangle .
$$

We get

$$
\Lambda\left[x_{\rho}\right] /\left(\mathrm{QSR}+\operatorname{Lin}+\left\langle x_{\mathrm{top}}\right\rangle\right) \stackrel{\sim}{\longrightarrow} \Lambda \otimes\left(H^{2 *}(X, \mathbb{C}) /\left\langle c_{\text {top }}(\mathcal{E})\right\rangle\right) .
$$

Thus, $B / \bar{x}_{\text {top }} B$ is a flat $\Lambda$-module. Its rank can be computed, and is equal to : $\operatorname{dim}_{\mathbb{C}} H^{2 *}(Y) / c_{\text {top }}(\mathcal{E}) H^{2 *}(Y)=\operatorname{dim} H^{2 *}(Y)-\operatorname{dim}_{\mathbb{C}} \operatorname{Im} m_{c_{\text {top }}}=\operatorname{dim}_{\mathbb{C}} \operatorname{ker} m_{c_{\text {top }}}$.

Restricting the exact sequence (3.41) to $\bar{U}$, and using the isomorphism $B^{\text {Res }} \stackrel{\sim}{\longrightarrow} \bar{x}_{\text {top }} B$ we get : $\left.\left(B^{\text {Res }}\right)\right|_{\bar{U}}$ is a flat module of rank $\left(\operatorname{dim} H^{2 *}(Y)-\operatorname{dim} \operatorname{ker} m_{c_{t o p}}\right)$ over $\bar{U}$.

\section{GKZ systems, quotient ideals and residual $\mathcal{D}$-modules}

GKZ systems were defined and studied by Gelfand-Kapranov-Zelevinskin in the end of the eighties (cf. [GGZ87], [GZK88], [GZK89] and [GKZ90]). Nevertheless, our approach is closer to the one of [Giv95], [Giv98], [CK99, §5.5.3 and §11.2] or [Iri09].

Here we define the GKZ ideal and the quotient GKZ ideal associated to $(X, \mathcal{E})$. This gives us two differential modules, which will be compared (in Section 5) to the twisted and reduced Quantum $\mathcal{D}$-modules of Section 2.

Consider, as above, the toric variety $X$ endowed with the $k$ toric divisors $L_{1}, \ldots, L_{k}$ such that $\mathcal{L}_{i}=\mathcal{O}\left(L_{i}\right)$. 
Notation 4.1. - Let $d$ be a class of $H_{2}(X, \mathbb{Z})$. We put

$$
\begin{array}{ll}
\forall \theta \in \Sigma(1), & d_{\theta}:=D_{\theta} \cdot d=\int_{d} D_{\theta}=\int_{d}\left[D_{\theta}\right] \\
\forall i \in\{1, \ldots, k\} & d_{L_{i}}:=L_{i} \cdot d=\int_{d} L_{i}=\int_{d} c_{1}\left(\mathcal{L}_{i}\right)
\end{array}
$$

Also recall that, for any real number $a$, we put $a^{+}=\max (a, 0), a^{-}=\max (-a, 0)$ so that : $a=a^{+}-a^{-}$.

Consider the non-commutative ring :

$$
\mathbb{D}:=\mathbb{C}\left[q_{1}^{ \pm 1}, \ldots, q_{r}^{ \pm 1}, z\right]\left\langle z \delta_{q_{1}}, \ldots, z \delta_{q_{r}}, z \delta_{z}\right\rangle .
$$

For simplicity, we will write $\mathbb{D}=\mathbb{C}\left[q^{ \pm}, z\right]\left\langle z \delta_{q}, z \delta_{z}\right\rangle$.

Notation 4.2. - (Quantization) Recall that $T_{1}, \ldots, T_{r}$ is a fixed basis of $H^{2}(X)$. To any class $\tau=\sum_{a=1}^{r} t_{a} T_{a} \in H^{2}(X)$ we associate the operator

$$
\widehat{\tau}:=\sum_{a=1}^{r} t_{a} z \delta_{q_{a}} \in \mathbb{D}
$$

In the same way, if $\mathcal{L}$ is a line bundle or a divisor we write $\widehat{\mathcal{L}}:=\widehat{c_{1}(\mathcal{L})}$. Finally put :

$$
\widehat{c}_{\text {top }}:=\prod_{i=1}^{k} \widehat{\mathcal{L}}_{i} \in \mathbb{D} \text {. }
$$

Definition 4.3. - (GKZ-ideal, and quotient ideal with respect to $\widehat{c}_{\text {top }}$ )

1. The GKZ-ideal $\mathbb{G}$ associated to $\left(\Sigma, \mathcal{L}_{1}, \ldots, \mathcal{L}_{k}\right)$ is the left ideal generated by the operators $\square_{d}, d \in H_{2}(X, \mathbb{Z})$, and $\widehat{\mathfrak{E}}$ :

$$
\begin{aligned}
\square_{d} & :=\prod_{i=1}^{k} \prod_{\nu=1}^{d_{L_{i}}^{-}}\left(\widehat{\mathcal{L}}_{i}+\nu z\right) \prod_{\theta \in \Sigma(1)} \prod_{\nu=0}^{d_{\theta}^{+}-1}\left(\widehat{D}_{\theta}-z \nu\right)- \\
& q^{d} \prod_{i=1}^{k} \prod_{\nu=1}^{d_{L_{i}}^{+}}\left(\widehat{\mathcal{L}}_{i}+\nu z\right) \prod_{\theta \in \Sigma(1)} \prod_{\nu=0}^{d_{\theta}^{-}-1}\left(\widehat{D}_{\theta}-z \nu\right) \quad\left(d \in H_{2}(X, \mathbb{Z})\right) \\
\widehat{\mathfrak{E}} & :=z \delta_{z}+c_{1}\left(\widehat{\mathcal{\mathcal { T }}_{X} \otimes \mathcal{E}^{\vee}}\right)
\end{aligned}
$$

where we use Notation 4.1 and $q^{d}=\prod_{a=1}^{r} q_{a}^{\int_{d} T_{a}}=\prod_{a=1}^{r} q_{a}^{d_{a}}\left(d=\sum_{a=1}^{r} d_{a} B_{a}\right)$.

2. The quotient ideal Quot $\left(\widehat{c}_{\text {top }}, \mathbb{G}\right)$ of $\mathbb{G}$ with respect to $\widehat{c}_{\text {top }}$, is the left ideal of $\mathbb{D}$ generated by :

$$
\left\{P \in \mathbb{D} \mid \widehat{c}_{\text {top }} P \in \mathbb{G}\right\} .
$$

Notice that, unlike the commutative case, the set $\left\{P \in \mathbb{D} \mid \widehat{c}_{\text {top }} P \in \mathbb{G}\right\}$ is not an ideal, but only a $\mathbb{C}[z]$-module ${ }^{(3)}$. However, it contains the ideal $\mathbb{G}$ and should be seen as a bigger system of equations.

Definition 4.4. - (GKZ-module, and residual module with respect to $\left.\widehat{c}_{\text {top }}\right)$

1. The GKZ module associated to $\left(\Sigma, \mathcal{L}_{1}, \ldots, \mathcal{L}_{k}\right)$ is the left quotient $\mathbb{D}$-module

$$
\mathbb{M}:=\mathbb{D} / \mathbb{G} \text {. }
$$

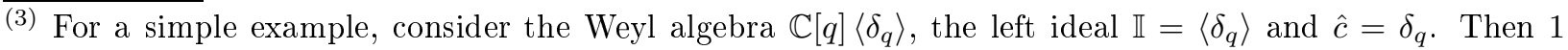
satisfies $\hat{c} .1 \in \mathbb{I}$, hence $\operatorname{Quot}(\hat{c}, \mathbb{I})=\mathbb{C}[q]\left\langle\delta_{q}\right\rangle$ and $q \in \operatorname{Quot}(\hat{c}, \mathbb{I})$. However, $\hat{c} . q=\delta_{q} q=q \delta_{q}+q$ is not in $\mathbb{I}$ (it would imply that $q$ is in $\mathbb{I}$, which is impossible for degree reasons).
} 
2. The residual GKZ-module $\mathbb{M}^{\text {res }}$ is the left quotient $\mathbb{D}$-module

$$
\mathbb{M}^{\text {res }}:=\mathbb{D} / \operatorname{Quot}\left(\widehat{c}_{\text {top }}, \mathbb{G}\right)
$$

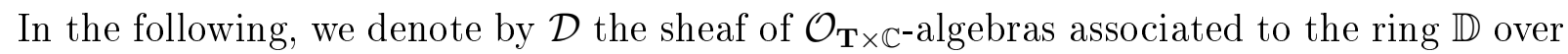

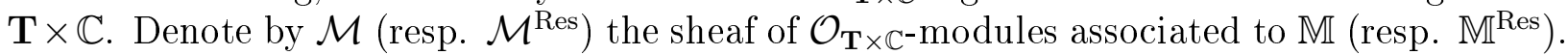

These $\mathcal{D}$-modules may not be coherent over the whole set $\mathbf{T} \times \mathbb{C}$. However, considering the freeness neighborhood $U$ defined in Notation 3.34, we have :

Theorem 4.5. - Let $\mathcal{L}_{1}, \ldots, \mathcal{L}_{k}$ be globally generated line bundles such that $\left(\omega_{X} \otimes \mathcal{L}_{1} \otimes\right.$ $\left.\cdots \otimes \mathcal{L}_{k}\right)^{\vee}$ is a nef line bundle. The restricted sheaves $\left.\mathcal{M}\right|_{U \times \mathbb{C}}$ and $\left.\mathcal{M}^{\mathrm{res}}\right|_{U \times \mathbb{C}}$ are coherent $\mathcal{O}_{U \times \mathbb{C}}-$ modules.

Proof. - If $\mathcal{M}$ is coherent then $\mathcal{M}^{\text {res }}$ is also coherent : the surjective morphism $\mathcal{M} \rightarrow \mathcal{M}^{\text {res }}$ implies that $\mathcal{M}^{\text {res }}$ is finitely generated.

Recall from Definition 4.4 that $\mathbb{M}=\mathbb{D} / \mathbb{G}$. This module $\mathbb{M}$ is isomorphic, as a $\mathbb{C}\left[q^{ \pm}, z\right]$ module, to

$$
\mathbb{M}^{\prime}:=\mathbb{D}^{\prime} / \mathbb{G}^{\prime}
$$

where $\mathbb{D}^{\prime}:=\mathbb{C}\left[q^{ \pm}, z\right]\left\langle z \delta_{q}\right\rangle$ and $\mathbb{G}^{\prime}=\left\langle\square_{d}, d \in H_{2}(X, \mathbb{Z})\right\rangle$ : the Euler operator $\widehat{\mathfrak{E}}$ of the ideal $\mathbb{G}$ enables us to remove $z \delta_{z}$ in the quotient.

Hence, we are led to show that the sheaf $\mathcal{M}^{\prime}$ associated to $\mathbb{M}^{\prime}$ and restricted to $U \times \mathbb{C}$ is $\mathbb{C}\left[q^{ \pm}, z\right]$-coherent. For a classical differential modules, one could find a good filtration and show that the characteristic variety is supported by the zero section of the cotangent bundle ( $c f$. [RS10, §3] and [Sab05, Proposition 1.2.8]). Since $\mathbb{M}^{\prime}$ is a $\mathbb{D}^{\prime}$-module (and not a $\mathbb{C}\left[q^{ \pm}, z\right]\left\langle\partial_{q}, \partial_{z}\right\rangle$-module), we will rewrite the classical proof ( $c f$. for instance [HTT08, Proposition 2.2.5]) in our case.

Let us define the following increasing filtration of $\mathbb{D}^{\prime}$ :

$$
F_{k} \mathbb{D}^{\prime}:=\left\{P \in \mathbb{D}^{\prime} \mid P\left(q, z, z \delta_{q}\right)=\sum_{\substack{\alpha \in \mathbb{N}^{r} \\|\alpha| \leq k}} P_{\alpha}(q, z)\left(z \delta_{q}\right)^{\alpha}\right\}
$$

where $\left(z \delta_{q}\right)^{\alpha}:=\left(z \delta_{q_{1}}\right)^{\alpha_{1}} \cdots\left(z \delta_{q_{r}}\right)^{\alpha_{r}}$. One can easily check that this filtration satisfies the following properties

(a) $F_{k} \mathbb{D}^{\prime}=0$ for $k<0$,

(b) $\cup_{k \in \mathbb{N}} F_{k} \mathbb{D}^{\prime}=\mathbb{D}^{\prime}$,

(c) for any $k, \ell$ in $\mathbb{N}$, we have $\left(F_{k} \mathbb{D}^{\prime}\right) \cdot\left(F_{\ell} \mathbb{D}^{\prime}\right)=F_{k+\ell} \mathbb{D}^{\prime}$,

(d) for any $k$ in $\mathbb{N}, F_{k} \mathbb{D}^{\prime}$ is a free $\mathbb{C}\left[q^{ \pm}, z\right]$-module,

(e) for any $P$ in $F_{k} \mathbb{D}^{\prime}$ and for any $Q$ in $F_{\ell} \mathbb{D}^{\prime},[P, Q]$ is in $F_{k+\ell-1} \mathbb{D}^{\prime}$.

Let gr $\mathbb{D}^{\prime}$ be the graduated ring of $\mathbb{D}^{\prime}$ defined by the filtration $F$. Property (e) proves that $\operatorname{gr} \mathbb{D}^{\prime}$ is commutative. For $a$ in $\{1, \ldots, r\}$, denote by $y_{a}$ the class of $z \delta_{q_{a}}$ in $\operatorname{gr} \mathbb{D}^{\prime}$, then gr $\mathbb{D}^{\prime}$ is isomorphic to $\mathbb{C}\left[q^{ \pm}, z\right]\left[y_{1}, \ldots, y_{r}\right]$. Let $P\left(q, z, z \delta_{q}\right):=\sum_{\alpha \in \mathbb{N}^{r}} P_{\alpha}(q, z)\left(z \delta_{q}\right)^{\alpha}$ in $\mathbb{D}^{\prime}$, then its class in gr $\mathbb{D}^{\prime}$, denoted by $\sigma(P)$, is

$$
\sigma(P)=\sum_{\substack{\alpha \in \mathbb{N}^{r} \\|\alpha|=\operatorname{deg} P}} P_{\alpha}(q, z) y^{\alpha}
$$

where $y^{\alpha}:=y_{1}^{\alpha_{1}} \cdots y_{r}^{\alpha_{r}}$.

We deduce an increasing filtration on $\mathbb{M}^{\prime}$ defined by

$$
F_{k} \mathbb{M}^{\prime}:=F_{k} \mathbb{D}^{\prime} / \mathbb{G}_{k}^{\prime}
$$

where $\mathbb{G}_{k}^{\prime}:=F_{k} \mathbb{D}^{\prime} \cap \mathbb{G}^{\prime}$. We have :

1. $F_{k} \mathbb{M}^{\prime}=0$ for $k<0$, 
2. $\cup_{k \in \mathbb{N}} F_{k} \mathbb{M}^{\prime}=\mathbb{M}^{\prime}$,

3. for any $k$ in $\mathbb{N}, F_{k} \mathbb{M}^{\prime}$ is a coherent $\mathbb{C}\left[q^{ \pm}, z\right]$-module as it is a finitely generated module over the Noetherian ring $\mathbb{C}\left[q^{ \pm}, z\right]$,

4. for any $k, \ell$ in $\mathbb{N}$, we have $\left(F_{k} \mathbb{D}^{\prime}\right) \cdot\left(F_{\ell} \mathbb{M}^{\prime}\right)=F_{k+\ell} \mathbb{M}^{\prime}$.

For classical differential module, the last two properties mean that the filtration $\left(F_{k} \mathbb{M}\right)_{k \in \mathbb{N}}$ is good. Since we have

$$
\operatorname{gr} \mathbb{M}^{\prime}=\operatorname{gr} \mathbb{D}^{\prime} / \operatorname{gr} \mathbb{G}^{\prime},
$$

we deduce that the annihilator $\mathrm{Ann}_{\mathrm{gr}} \mathbb{D}^{\prime} \operatorname{gr} \mathbb{M}^{\prime}$ is $\operatorname{gr} \mathbb{G}^{\prime}$.

In order to use Lemma 4.8, we sheafify everything. We will denote by calligraphic letters for the sheaves associated to the $\mathbb{C}\left[q^{ \pm}, z\right]$-modules and restricted to $U \times \mathbb{C}$. Consider the ideal sheaf $\mathcal{I}:=\left\langle y_{1}, \ldots, y_{r}\right\rangle$ in gr $\mathbb{D}^{\prime}$.

By Lemma 4.8, there exists ${ }^{(4)} m_{0}$ in $\mathbb{N}$ such that $\mathcal{I}^{m_{0}}$ is a subsheaf of gr $\mathbb{G}^{\prime}$. Let $P$ be a section of $F_{k} \mathbb{M}^{\prime}$ for $k \leq m_{0}$. By property (4), for $\alpha$ in $\mathbb{N}^{r}$ such that $|\alpha|=m_{0}$, we have $\left(z \delta_{q}\right)^{\alpha} P$ is in $F_{k+m_{0}} \mathbb{M}^{\prime}$. But we have $\sigma\left(\left(z \delta_{q}\right)^{\alpha}\right)=y^{\alpha} \in \mathcal{I}^{m_{0}} \subset \operatorname{gr} \mathbb{G}^{\prime}$. This implies that the class of $\left(z \delta_{q}\right)^{\alpha} P$ in gr $\mathbb{M}^{\prime}$ is zero. We deduce that $\left(z \delta_{q}\right)^{\alpha} P$ in $F_{k+m_{0}-1} \mathbb{M}^{\prime}$. The property (4) implies that for any $k$ in $\mathbb{N}$ we have

$$
\begin{aligned}
F_{m_{0}+k} \mathbb{M}^{\prime} & =\left(F_{m_{0}} \mathbb{D}^{\prime}\right) \cdot F_{k} \mathbb{M}^{\prime} \\
& =\left(\sum_{\substack{\alpha \in \mathbb{N}^{r} \\
|\alpha|=m_{0}}} \mathcal{O}_{U \times \mathbb{C}}\left(z \delta_{q}\right)^{\alpha}\right) \cdot F_{k} \mathbb{M}^{\prime}+\left(\sum_{\substack{\alpha \in \mathbb{N}^{r} \\
|\alpha|<m_{0}}} \mathcal{O}_{U \times \mathbb{C}}\left(z \delta_{q}\right)^{\alpha}\right) \cdot F_{k} \mathbb{M}^{\prime} \\
& \subset F_{k+m_{0}-1} \mathbb{M}^{\prime} .
\end{aligned}
$$

We deduce that the increasing filtration $F_{k} \mathbb{M}^{\prime}$ is stationary after $m_{0}$. Property (2) implies that $F_{m_{0}} \mathbb{M}^{\prime}=\mathbb{M}^{\prime}$ and Property (3) implies the theorem.

The following Lemma is used in Theorem 4.5. We use the notations defined along the proof of this theorem : gr $\mathbb{D}^{\prime}=\mathbb{C}\left[q_{1}^{ \pm}, \ldots, q_{r}^{ \pm}, z\right]\left[y_{1}, \ldots, y_{r}\right]$ and $\sigma$ is the symbol. Recall that the characteristic variety of $\mathbb{M}$ is the algebraic variety $C$ in Spec gr $\mathbb{D}^{\prime}$ defined by the ideal : $\sqrt{\mathrm{Ann}_{\mathrm{gr}} \mathbb{D}^{\prime} \text { gr } \mathbb{M}^{\prime}}$. There is a natural morphism : Spec gr $\mathbb{D}^{\prime} \longrightarrow \mathbf{T} \times \mathbb{C}$, where $\mathbf{T}=\operatorname{Spec} \mathbb{C}\left[q^{ \pm}\right]$, and we get a cartesian diagram :

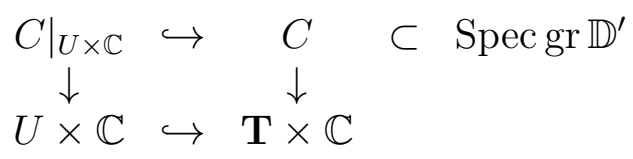

Lemma 4.8. - Assume that $\left(\omega_{X} \otimes \mathcal{L}_{1} \otimes \cdots \otimes \mathcal{L}_{k}\right)^{\vee}$ is a nef line bundle. Let $U$ be the open subset of $\mathbf{T}$ defined in Notation 3.34. The characteristic variety $\left.C\right|_{U \times \mathbb{C}}$ is the zero section of $\left.\operatorname{gr} \mathbb{D}^{\prime}\right|_{U \times \mathbb{C}} \rightarrow U \times \mathbb{C}$ defined by the ideal $\left\langle y_{1}, \ldots, y_{r}\right\rangle$.

Proof. - In order to connect the definitions of $U$ and gr $\mathbb{D}^{\prime}$, we consider the ring $\mathbb{C}\left[q^{ \pm}, z\right]\left[x_{\rho}, \rho \in \Delta(1)\right]$. There is a natural surjective morphism of $\mathbb{C}\left[q^{ \pm}, z\right]$-algebra :

$$
\begin{aligned}
\alpha: \mathbb{C}\left[q^{ \pm}, z\right]\left[x_{\rho}, \rho \in \Delta(1)\right] & \longrightarrow \operatorname{gr} \mathbb{D}^{\prime}=\mathbb{C}\left[q^{ \pm}, z\right]\left[y_{1}, \ldots, y_{r}\right] \\
x_{\rho} & \longmapsto \begin{cases}\sum_{a=1}^{r} D_{\rho}^{a} y_{a} & \text { if } \rho \in \Delta_{(1)}^{\text {base }} \\
-\left(\sum_{a=1}^{r} D_{\rho}^{a} y_{a}\right) & \text { if } \rho \in \Delta_{(1)}^{\text {v.b. }}\end{cases}
\end{aligned}
$$

Where the $D_{\rho}^{a}$ are numbers defined by : $\left[D_{\rho}\right]=\sum_{a=1}^{r} D_{\rho}^{a} T_{a}$ in $H^{2}(Y, \mathbb{Z})$. Note that since, in $\square_{d}$, the sign in front of $\mathcal{D}_{\rho}$ in is not the same for rays coming from the bases or from the line

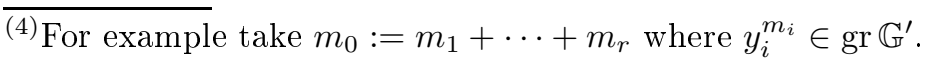


bundles $\mathcal{L}_{i}$, the definition of $\alpha\left(x_{\rho}\right)$ admit two cases. Actually, $c_{1}\left(\mathcal{L}_{i}\right)=-\left[D_{\rho_{i}}\right]$ for $\rho_{i} \in \Delta^{\text {v.b. }}(1)$ which create this additional sign. We refer the reader to [CK99] and to the Erratum to Proposition 5.5.4 for definitions of $\square_{d}$ with conventional signs).

The kernel of this morphism is the linear ideal Lin generated by the $Z_{u}, u \in M^{\prime}: Z_{u}=$ $\sum_{\rho \in \Delta(1)}\left\langle u, v_{\rho}\right\rangle x_{\rho}$ where $v_{\rho}$ is the generating vector of $\rho \cap N^{\prime}$. We get an isomorphism :

$$
\bar{\alpha}: \mathbb{C}\left[q^{ \pm}, z\right]\left[x_{\rho}, \rho \in \Delta(1)\right] / \operatorname{Lin} \longrightarrow \mathbb{C}\left[q^{ \pm}, z\right]\left[y_{1}, \ldots, y_{r}\right] .
$$

The characteristic variety is contained in the closed subset $K_{1}^{\prime}$ of Spec gr $\mathbb{D}^{\prime}$ defined by the ideal

$$
J_{1}=\left\langle\sigma\left(\square_{d}\right), d \in H_{2}(X, \mathbb{Z})\right\rangle \subset \mathbb{C}\left[q^{ \pm}, z\right]\left[y_{1}, \ldots, y_{r}\right] .
$$

Let $d$ be in $H_{2}(X, \mathbb{Z})$. We check that $\sigma\left(\square_{d}\right)=\bar{\alpha}\left(R_{d}^{\infty}\right)$ where $R_{d}^{\infty}$ is the polynomial :

$$
R_{d}^{\infty}:= \begin{cases}x^{d^{+}} & \text {if } \sum_{\rho \in \Delta(1)} d_{\rho}<0 \\ x^{d^{+}}-q^{d} x^{d^{-}} & \text {if } \sum_{\rho \in \Delta(1)} d_{\rho}=0 .\end{cases}
$$

Which lead us to consider the ideal :

$$
J_{2}=\left\langle R_{d}^{\infty}, d \in H_{2}(X, \mathbb{Z}) ; Z_{u}, u \in M^{\prime}\right\rangle \subset \mathbb{C}\left[q^{ \pm}, z\right]\left[x_{\rho}, \rho \in \Delta(1)\right],
$$

Considering $\mathbb{C}\left[q^{ \pm}, z\right]\left[x_{\rho}, \rho \in \Delta(1)\right]$ and $\mathbb{C}\left[q^{ \pm}, z\right]\left[y_{1}, \ldots, y_{r}\right]$ as graded $\mathbb{C}\left[q^{ \pm}, z\right]$-algebras (with $\operatorname{deg}\left(x_{\rho}\right)=1$ and $\operatorname{deg}\left(y_{a}\right)=1$ ), The morphism $\alpha$ defined above is a graded morphism. Ideals $J_{1}$ and $J_{2}$ both are homogeneous ideals, and $\alpha\left(J_{2}\right)=J_{1}$.

Let $K_{1}$ (resp. $\left.K_{2}\right)$ be the closed subscheme of the projective scheme Proj $\mathbb{C}\left[q^{ \pm}, z\right]\left[y_{1}, \ldots, y_{r}\right]$ (resp. Proj $\mathbb{C}\left[q^{ \pm}, z\right]\left[x_{\rho}, \rho \in \Delta(1)\right]$ ) defined by $J_{1}$ (resp. $J_{2}$ ). Also put :

$$
\begin{array}{r}
\pi_{1}: \operatorname{Proj} \mathbb{C}\left[q^{ \pm}, z\right]\left[y_{1}, \ldots, y_{r}\right] \longrightarrow \operatorname{Spec} \mathbb{C}\left[q^{ \pm}, z\right]=\mathbf{T} \times \mathbb{C} \\
\pi_{2}: \operatorname{Proj} \mathbb{C}\left[q^{ \pm}, z\right]\left[x_{\rho}, \rho \in \Delta(1)\right] \longrightarrow \operatorname{Spec} \mathbb{C}\left[q^{ \pm}, z\right]=\mathbf{T} \times \mathbb{C}
\end{array}
$$

the natural projections.

By Definition of $U$ (Notation 3.34), and using Remark 3.35, we find : $(\mathbf{T} \times \mathbb{C}) \backslash \pi_{2}\left(K_{2}\right)=$ $U \times \mathbb{C}$. Since the isomorphism $\bar{\alpha}$ satisfies $\bar{\alpha}\left(J_{2}\right)=J_{1}$, we have

$$
(\mathbf{T} \times \mathbb{C}) \backslash \pi_{1}\left(K_{1}\right)=U \times \mathbb{C} .
$$

Consider now the affine space Spec $\mathbb{C}\left[q^{ \pm}, z\right]\left[y_{1}, \ldots, y_{r}\right]$ (before projectivization) and the closed subvariety $K_{1}^{\prime}$ defined by $J_{1}$. By definition, the characteristic variety $C$ is the reduced scheme of $K_{1}^{\prime}$.

The ideal $J_{1}$ is contained in $\left\langle y_{1}, \ldots, y_{r}\right\rangle$ (this can be checked by considering the polynomials $R_{d}^{\infty}$ and the relation $\left.\alpha\left(R_{d}^{\infty}\right)=\sigma\left(\square_{d}\right)\right)$. It follows that the zero section of the morphism :

$$
\pi_{1}^{\prime}: \operatorname{Spec} \mathbb{C}\left[q^{ \pm}, z\right]\left[y_{1}, \ldots, y_{r}\right] \longrightarrow \operatorname{Spec} \mathbb{C}\left[q^{ \pm}, z\right]=\mathbf{T} \times \mathbb{C}
$$

is contained in the support $K_{1}^{\prime}$.

On the other hand, the relation (4.9) implies that $\pi_{1}^{-1}(U) \cap K_{1}=\varnothing$ which means that the support of $\pi_{1}^{\prime-1}(U \times \mathbb{C}) \cap K_{1}^{\prime}$ is contained in the zero section of $\pi_{1}^{\prime}$. Indeed, the ideal $\left\langle y_{1}, \ldots, y_{r}\right\rangle$ is the irrelevant ideal of the graded ring $\mathbb{C}\left[q^{ \pm}, z\right]\left[y_{1}, \ldots, y_{r}\right]$.

This shows that the support of $\pi_{1}^{\prime-1}(U \times \mathbb{C}) \cap K_{1}^{\prime}$, i.e., the characteristic variety $C$ of $G$ restricted to $U \times \mathbb{C}$, is equal to the zero section of $\left.\pi_{1}^{\prime}\right|_{U}$.

Theorem 4.10. - Let $\mathcal{L}_{1}, \ldots, \mathcal{L}_{k}$ be globally generated line bundles such that $\left(\omega_{X} \otimes \mathcal{L}_{1} \otimes\right.$ $\left.\cdots \otimes \mathcal{L}_{k}\right)^{\vee}$ is nef. Let $U$ be the open subset of $\mathbf{T}$ defined in Notation 3.34. The $\mathcal{D}$-module $\left.\mathcal{M}\right|_{U \times \mathbb{C}}$ is locally free of rank $\operatorname{dim} H^{2 *}(X)$. 
Proof. - The following proof is similar to the proof of Theorem 2.14 of [RS10]. Modifications as to be made in order to take care of the twisted fan and the $q$ 's variables.

Theorem 4.5 implies that $\left.\mathcal{M}\right|_{U \times \mathbb{C}}$ is a coherent $\mathcal{O}_{U \times \mathbb{C}-\text { modules. By standard arguments }}$ (see for instance Theorem 1.4.10 of [HTT08]), for $z \neq 0$ this implies that $\left.\mathcal{M}\right|_{U \times \mathbb{C}^{*}}$ is locally free. It is enough to prove that $\mathcal{M} / z \mathcal{M}$ is locally free of rank $\operatorname{dim} H^{2 *}(X)$ and that the locally free sheaf $\left.\mathcal{M}\right|_{U \times \mathbb{C}^{*}}$ is of the same rank.

Step 1. Show that $\mathcal{M} / z \mathcal{M}$ is locally free of rank $H^{2 *}(X)$.

Let $B$ be the Batyrev ring $\Lambda\left[x_{\rho}\right] /\langle$ QSR $+\operatorname{Lin}\rangle$. Localizing the ring $\Lambda$ by inverting $Q^{d}, d \in$ $\mathrm{NE}(X)_{\mathbb{Z}}$, we obtain the ring $\mathbb{C}\left[q^{ \pm}\right]=\mathbb{C}\left[q_{1}^{ \pm}, \ldots, q_{r}^{ \pm}\right]$. Using Notation 3.2 , there is an isomorphism of $\mathbb{C}\left[q^{ \pm}\right]$-algebra :

$B \otimes \mathbb{C}\left[q^{ \pm}\right]=\mathbb{C}\left[q^{ \pm}, x_{\rho}\right] /\langle\mathrm{QSR}+\operatorname{Lin}\rangle \longrightarrow \mathbb{M} / z \mathbb{M}=\mathbb{D}^{\prime} /\left(\langle z\rangle+\mathbb{G}^{\prime}\right)$

$$
x_{\rho} \longmapsto \begin{cases}\sum_{a=1}^{r} D_{\rho}^{a} z \delta_{q_{a}} & \text { if } \rho \in \Delta^{\text {base }}(1)=\left\{\rho_{\theta}, \theta \in \Sigma(1)\right\} \\ \sum_{a=1}^{r} L_{i}^{a} z \delta_{q_{a}} & \text { if } \rho=\rho_{L_{i}} \in \Delta^{\text {v.b. }}(1)=\left\{\rho_{L_{1}}, \ldots, \rho_{L_{k}}\right\}\end{cases}
$$

(where $\left[L_{i}\right]=\sum_{a} L_{i}^{a} T_{a}$ ). By Theorem 3.26, the Batyrev ring $B$ is locally free of rank $\operatorname{dim} H^{2 *}(X)$ over the neighbourhood $\bar{U}$. Hence $\mathcal{M} / z \mathcal{M}$ is locally free of rank $\operatorname{dim} H^{2 *}(X)$ over the open subset $U=\bar{U} \cap \mathbf{T}$.

Step 2. We use the notation of the beginning of the proof of Theorem 4.5. On $z \neq 0$, we show that the locally free sheaf $\left.\mathcal{M}\right|_{U \times \mathbb{C}^{*}}$ is of rank $H^{2 *}(X)$. To prove this, we will use 2 substeps.

2.1 Show that the module $\mathbb{M}:=\mathbb{D} / \mathbb{G} \simeq \mathbb{M}^{\prime}:=\mathbb{D}^{\prime} / \mathbb{G}^{\prime}$ (see (4.6)) is isomorphic to a classical GKZ-system of Adolphson (see [Ado94]). Notice that most of this step is done for any $z \in \mathbb{C}$ (included $z=0$ ).

2.2 We compute that the rank is $\operatorname{dim} H^{2 *}(X)$ at one point using corollary 5.11 of [Ado94].

Step 2.1 We first write the GKZ system $\mathbb{M}^{\prime}:=\mathbb{D}^{\prime} / \mathbb{G}^{\prime}$ in a more classical way in view of Adolphson's result ([Ado94]).

Let $\left\{\lambda_{\rho}, \rho \in \Delta(1)\right\}$ be a set of indeterminates. Consider the following non commutative rings :

- $\mathbb{A}_{1}:=\mathbb{C}\left[\lambda^{ \pm}, z\right]\left\langle z \partial_{\lambda}\right\rangle=\mathbb{C}\left[\lambda_{\rho}, \lambda_{\rho}^{-1}, \rho \in \Delta(1), z\right]\left\langle z \partial_{\lambda_{\rho}}, \rho \in \Delta(1)\right\rangle$. where the relations are : $z \partial_{\lambda_{\rho}} . \lambda_{\rho}=\lambda_{\rho} . z \partial_{\lambda_{\rho}}+z$ and all the other variables are commuting;

- $\mathbb{A}_{2}:=\mathbb{C}\left[q^{ \pm}, z\right]\left\langle z \delta_{\lambda}\right\rangle=\mathbb{C}\left[q_{a}, q_{a}^{-1}, a \in\{1, \ldots, r\}, z\right]\left\langle z \delta_{\lambda_{\rho}}, \rho \in \Delta(1)\right\rangle$. where the relations are : $z \delta_{\lambda_{\rho}} . q_{a}=q_{a} . z \delta_{\lambda_{\rho}}+D_{\rho}^{a} z q_{a}$ where the $D_{\rho}^{a}$ are numbers defined by : $\left[D_{\rho}\right]=$ $\sum_{a=1}^{r} D_{\rho}^{a} T_{a}$ in $H^{2}(Y, \mathbb{Z})$.

In view of $[\mathbf{C K 9 9}]$ and its Erratum to proposition 5.5.4, we put in $\mathbb{A}_{1}: \ell:=\prod_{\rho \in \Delta \text { v.b. }} \lambda_{\rho}$. There exists two morphisms of noncommutative $\mathbb{C}[z]$-algebras, $f$ and $g$ defined by :

$$
\begin{aligned}
f: \mathbb{A}_{2} & \longleftrightarrow \mathbb{A}_{1} \\
z \delta_{\lambda_{\rho}} & \longmapsto \ell^{-1}\left(\lambda_{\rho} . z \partial_{\lambda_{\rho}}\right) \ell= \begin{cases}\lambda_{\rho} . z \partial_{\lambda_{\rho}} & \text { if } \rho \in \Delta^{\text {base }}(1) \\
\lambda_{\rho} . z \partial_{\lambda_{\rho}}+z & \text { if } \rho \in \Delta^{\text {v.b. }}(1)\end{cases} \\
q_{a} & \longmapsto(-1)^{c_{1}(\mathcal{E}) \cdot B_{a}} \lambda^{d}=\prod_{\substack{\text { base } \\
\rho \in \Delta(1)}} \lambda_{\rho}^{D_{\rho}^{a}} \cdot \prod_{\substack{\text { v.b. } \\
\rho \in \Delta(1)}}\left(-\lambda_{\rho}\right)^{D_{\rho}^{a}},
\end{aligned}
$$

and 


$$
\begin{aligned}
g: \mathbb{A}_{2} & \longrightarrow \mathbb{D}^{\prime} \\
z \delta_{\lambda_{\rho}} & \longmapsto \begin{cases}\widehat{D}_{\rho}:=\sum_{a=1}^{r} D_{\rho}^{a} z \delta_{q_{a}}, & \text { if } \rho \in \Delta^{\text {base }}(1) \\
-\widehat{L}_{i}:=-\sum_{a=1}^{r} L_{\rho}^{a} z \delta_{q_{a}}, & \text { if } \rho_{i} \in \Delta_{(1)}^{\text {v.b. }}\end{cases}
\end{aligned}
$$

and $g\left(q_{a}\right)=q_{a}$. The minus sign comes because the fan $\Delta$ is the one of the dual bundle (see Proposition 3.3). The morphism $f$ is injective. The morphism $g$ is surjective and its kernel is the left ideal generated by the following set :

$$
\left\{\mathcal{Z}_{u}=\sum_{\rho \in \Delta(1)}\left\langle v_{\rho}, u\right\rangle z \delta_{\lambda_{\rho}}, u \in M^{\prime}\right\} \subset \mathbb{A}_{2} .
$$

The GKZ ideal can be defined in $\mathbb{A}_{1}$ and $\mathbb{A}_{2}$ :

In $\mathbb{A}_{1}$, set $\square_{d}^{\prime \prime}:=\left(z \partial_{\lambda}\right)^{d^{+}}-\left(z \partial_{\lambda}\right)^{d^{-}}$for any $d \in H_{2}(X, \mathbb{Z})$ and $\mathcal{Z}_{u}^{\prime}:=\sum_{\rho}\left\langle u, v_{\rho}\right\rangle \lambda_{\rho} z \partial_{\lambda_{\rho}}-$ $\langle u, \beta\rangle z$ for any $u \in M^{\prime}$, where $\beta$ is the constant vector $\left(0_{N},-1, \ldots,-1\right) \in N \times \mathbb{Z}^{k}$. The GKZ ideal is

$$
\mathbb{G}_{1}=\left\langle\square_{d}^{\prime \prime}, \mathcal{Z}_{u}^{\prime}, d \in H_{2}(X, \mathbb{Z}), u \in M^{\prime}\right\rangle \subset \mathbb{A}_{1} .
$$

In $\mathbb{A}_{2}$ set :

$$
\begin{aligned}
& \square_{d}^{\prime}:=\prod_{\substack{\text { bass } \\
\rho \in \Delta(1)}} \prod_{\nu=0}^{d_{\rho}^{+}-1}\left(z \delta_{\lambda_{\rho}}-\nu z\right) \prod_{\substack{\text { v.t. } \\
\rho \in \Delta(1)}} \prod_{\nu=1}^{d_{\rho}^{+}}\left(-z \delta_{\lambda_{\rho}}+\nu z\right) \\
& -q^{d} \prod_{\substack{\text { bass } \\
\rho \in \Delta(1)}}^{d_{\nu=0}^{-}-1}\left(z \delta_{\lambda_{\rho}}-\nu z\right) \prod_{\substack{\nu . \text {.. } \\
\rho \in(1)}} \prod_{\nu=1}^{d_{\rho}^{-}}\left(-z \delta_{\lambda_{\rho}}+\nu z\right),
\end{aligned}
$$

and $\mathcal{Z}_{u}:=\sum_{\rho}\left\langle u, v_{\rho}\right\rangle z \delta_{\lambda_{\rho}}$. The GKZ ideal in $\mathbb{A}_{2}$ is $\mathbb{G}_{2}:=\left\langle\square_{d}^{\prime}, \mathcal{Z}_{u}, d \in H_{2}(X, \mathbb{Z}), u \in M^{\prime}\right\rangle$.

As $g\left(z \delta_{\lambda_{\rho_{i}}}\right)=-\widehat{L}_{i}$ for any $\rho_{i} \in \Delta_{(1)}^{\text {v.b. }}$, we have :

$$
\begin{gathered}
f\left(\square_{d}^{\prime}\right)=\prod_{\substack{\text { base } \\
\rho \in \Delta(1)}} \lambda_{\rho}^{d_{\rho}^{+}} \prod_{\substack{\text { v.b. } \\
\rho \in \Delta(1)}}\left(-\lambda_{\rho}\right)^{d_{\rho}^{+}} \cdot \square_{d}^{\prime \prime} \quad f\left(\mathcal{Z}_{u}\right)=\mathcal{Z}_{u}^{\prime} \\
g\left(\square_{d}^{\prime}\right)=\square_{d}
\end{gathered}
$$

which gives $f\left(\mathbb{G}_{2}\right)=\mathbb{G}_{1}$ and $g\left(\mathbb{G}_{2}\right)=\mathbb{G}^{\prime}$. Passing to the quotient, the morphism $g$ gives an isomorphism :

$$
\mathbb{A}_{2} / \mathbb{G}_{2} \stackrel{\sim}{\longrightarrow} \mathbb{D}^{\prime} / \mathbb{G}^{\prime}=\mathbb{M}
$$

Moreover, viewing $\mathbb{C}\left[\lambda^{ \pm}, z\right]$ as a $\mathbb{C}\left[q^{ \pm}, z\right]$-algebra via the injective morphism

$$
\begin{aligned}
\varphi: \mathbb{C}\left[q^{ \pm}\right] & \longleftrightarrow \mathbb{C}\left[\lambda^{ \pm}\right] \\
q_{a} & \longmapsto(-1)^{c_{1}(\mathcal{E}) \cdot B_{a}} \lambda^{d}
\end{aligned}
$$

we get an isomorphism :

$$
\mathbb{A}_{2} \otimes_{\mathbb{C}\left[q^{ \pm}, z\right]} \mathbb{C}\left[\lambda^{ \pm}, z\right] \stackrel{\sim}{\longrightarrow} \mathbb{A}_{1}
$$

which sends $z \delta_{\rho}$ to $\lambda_{\rho} . z \partial_{q}$. This gives an isomorphism :

$$
\mathbb{A}_{2} / \mathbb{G}_{2} \otimes_{\mathbb{C}\left[q^{ \pm}, z\right]} \mathbb{C}\left[\lambda^{ \pm}, z\right] \stackrel{\sim}{\longrightarrow} \mathbb{A}_{1} / \mathbb{G}_{1} .
$$

In the following, we will write "the module is locally free over an open" meaning "the sheaf associated to the module is locally free". As $\varphi$ is injective, we have $\mathbb{A}_{2} / \mathbb{G}_{2} \simeq \mathbb{D}^{\prime} / \mathbb{G}^{\prime}$ is locally free at a point $\mathfrak{n}$ of $\operatorname{Spec} \mathbb{C}\left[q^{ \pm}, z\right]$ if and only $\mathbb{A}_{1} / \mathbb{G}_{1}$ is locally free at any point $\mathfrak{n}^{\prime}$ of $\operatorname{Spec} \mathbb{C}\left[\lambda^{ \pm}, z\right]$ contained in the fiber of the surjective morphism Spec $\mathbb{C}\left[\lambda^{ \pm}, z\right] \rightarrow \operatorname{Spec} \mathbb{C}\left[q^{ \pm}, z\right]$. 
Now we localize this $\mathbb{C}\left[q^{ \pm}, z\right]$-module above $z \neq 0$. By Theorem 4.5, the module $\mathbb{D}^{\prime} / \mathbb{G}^{\prime} \otimes_{\mathbb{C}\left[q^{ \pm}, z\right]} \mathbb{C}\left[q^{ \pm}, z^{ \pm}\right]$is locally free over $U \times \mathbb{C}^{*}$. By the above isomorphisms, the module $\mathbb{A}_{1} / \mathbb{G}_{1} \otimes_{\mathbb{C}\left[\lambda^{ \pm}, z\right]} \mathbb{C}\left[\lambda^{ \pm}, z^{ \pm}\right]$is also locally free over $\left(\left(\varphi^{\#}\right)^{-1}(U)\right) \times \mathbb{C}^{*}$ where $\varphi^{\#}:\left(\mathbb{C}^{*}\right)^{\Delta(1)} \rightarrow\left(\mathbb{C}^{*}\right)^{r}$ is the scheme morphism associated to $\varphi$ (see (4.13)). On the other hand, we have the isomorphism :

$$
\begin{aligned}
\mathbb{A}_{1} \otimes_{\mathbb{C}\left[\lambda^{ \pm}, z\right]} \mathbb{C}\left[\lambda^{ \pm}, z^{ \pm}\right] & \stackrel{\sim}{\mathbb{C}}\left[\lambda^{ \pm}, z^{ \pm}\right]\left\langle\partial_{\lambda}\right\rangle \\
\lambda_{\rho} & \longmapsto z \lambda_{\rho} \\
\left(z \partial_{\lambda_{\rho}}\right) & \longmapsto \partial_{\lambda_{\rho}} \\
z & \longmapsto z
\end{aligned}
$$

which sends $\square_{d}^{\prime \prime}=\left(z \partial_{\lambda}\right)^{d^{+}}-\left(z \partial_{\lambda}\right)^{d^{-}}$to $\square_{d}^{\prime \prime \prime}:=\partial_{\lambda}^{d^{+}}-\partial_{\lambda}^{d^{-}}$and $\mathcal{Z}_{u}^{\prime}$ to $z \cdot\left(\sum_{\rho}\left\langle u, v_{\rho}\right\rangle \lambda_{\rho} \partial_{\lambda_{\rho}}-\langle u, \beta\rangle\right)$. Put $\mathcal{Z}_{u}^{\prime \prime}:=\sum_{\rho}\left\langle u, v_{\rho}\right\rangle \lambda_{\rho} \partial_{\lambda_{\rho}}-\langle u, \beta\rangle$, the module $\mathbb{A}_{1} / \mathbb{G}_{1} \otimes_{\mathbb{C}\left[\lambda^{ \pm}, z\right]} \mathbb{C}\left[\lambda^{ \pm}, z^{ \pm}\right]$is isomorphic, as a $\mathbb{C}\left[\lambda^{ \pm}\right] \otimes_{\mathbb{C}} \mathbb{C}\left[z^{ \pm}\right]$-module to

$$
\mathbb{C}\left[\lambda^{ \pm}\right]\left\langle\partial_{\lambda}\right\rangle /\left\langle\square_{d}^{\prime \prime \prime}, \mathcal{Z}_{u}^{\prime \prime}\right\rangle \otimes_{\mathbb{C}} \mathbb{C}\left[z^{ \pm}\right] .
$$

We deduce that $\mathbb{C}\left[\lambda^{ \pm}\right]\left\langle\partial_{\lambda}\right\rangle /\left\langle\square_{d}^{\prime \prime \prime}, \mathcal{Z}_{u}^{\prime \prime}\right\rangle$ is locally free over $\left(\varphi^{\#}\right)^{-1}(U)$.

Step 2.2 Let us compute the rank of the locally free module $\mathbb{C}\left[\lambda^{ \pm}\right]\left\langle\partial_{\lambda}\right\rangle /\left\langle\square_{d}^{\prime \prime \prime}, \mathcal{Z}_{u}^{\prime \prime}\right\rangle$ over $\left(\varphi^{\#}\right)^{-1}(U)$ using Corollary 5.11 of [Ado94]. In view of the local freeness, we just need to compute the rank at one point.

Adolphson associates a Laurent polynomial to the module $\mathbb{C}\left[\lambda^{ \pm}\right]\left\langle\partial_{\lambda}\right\rangle /\left\langle\square_{d}^{\prime \prime \prime}, \mathcal{Z}_{u}^{\prime \prime}\right\rangle$, denoted by $f_{\lambda}$. We do not need to give the precise expression of $f_{\lambda}$ for the following. Corollary 5.11 of [Ado94] tells us that over the following set

$$
\left\{\left(\lambda_{\rho}\right) \in\left(\mathbb{C}^{*}\right)^{\Delta(1)} \mid f_{\lambda} \text { is non degenerated }\right\}
$$

the rank is $(n+k) ! \operatorname{Vol}\left(\Gamma_{\Delta}\right)$ where $\Gamma_{\Delta}$ is the convex hull in $N_{\mathbb{R}}^{\prime}$ of the points $\left\{0, v_{\rho}, \rho \in \Delta(1)\right\}$. This set is a nonempty Zariski open subset of $\left(\mathbb{C}^{*}\right)^{\Delta(1)}$. By density, this Zariski open intersects the Zariski open subset $\left(\varphi^{\#}\right)^{-1}(U)$ so that the rank is equal to $(n+k) ! \operatorname{Vol}\left(\Gamma_{\Delta}\right)$.

Denote by $\Gamma_{\Sigma}$ the convex hull in $N_{\mathbb{R}}$ of the points $\left\{0, v_{\rho}, \rho \in \Sigma(1)\right\}$. Show that $(n+$ $k) ! \operatorname{Vol}\left(\Gamma_{\Delta}\right)=n ! \operatorname{Vol}\left(\Gamma_{\Sigma}\right)=\operatorname{dim} H^{2 *}(X)$. When all the $L_{i}$ are nef, the fan $\Delta$ is convex, and 0 is a vertex of this convex hull. As the divisor $-K_{X}-\sum_{i=1}^{k} L_{i}$ is nef, then the vectors $\left(v_{1}, \ldots, v_{k}\right) \in N \times \mathbb{Z}^{k}$ defined by the toric divisors $L_{i}$ all are either vertices or contained in faces of $\Gamma_{\Delta}$ which do not contain 0 . Hence, $\Gamma_{\Delta}$ is a disjoint union of the simplexes $\Gamma_{\Delta}(\tau):=$ $\left(v_{1}, \ldots, v_{k}, v_{\rho_{\theta}}, \theta \in \tau\right)$ where $\tau$ is any simplex defined by generating vectors of the rays in $\Sigma$ (we use the notations of Section 3.1). The volume of a simplex $\Gamma_{\Delta}(\tau)$ can be computed by a determinant on these vectors which simplifies into the volume of $\Gamma_{\Sigma}(\tau):=\left(v_{\rho_{\theta}}, \theta \in \tau\right)$. Since the union of these simplexes is $\Gamma_{\Sigma}$, we have $(n+k) ! \operatorname{Vol}\left(\Gamma_{\Delta}\right)=n ! \operatorname{Vol}\left(\Gamma_{\Sigma}\right)$ which is exactly $\operatorname{dim} H^{2 *}(X)$.

Theorem 4.14. - Let $\mathcal{L}_{1}, \ldots, \mathcal{L}_{k}$ be ample line bundles such that $\left(\omega_{X} \otimes \mathcal{L}_{1} \otimes \cdots \otimes \mathcal{L}_{k}\right)^{\vee}$ is nef. Let $U$ be the open subset of $\mathbf{T}$ defined in Notation 3.34.

1. On $z=0$, the $\mathcal{O}_{U}$-module $\left.\left(\mathcal{M}^{\text {res }} / z \mathcal{M}^{\text {res }}\right)\right|_{U}$ is locally free of rank $\operatorname{dim}_{\mathbb{C}} \overline{H^{2 *}(X)}=$ $\operatorname{dim}_{\mathbb{C}} H^{2 *}(X)-\operatorname{dim}_{\mathbb{C}} \operatorname{ker}\left(m_{c_{\text {top }}}\right)$.

2. On $z \neq 0$, the $\left.\mathcal{O}_{U \times \mathbb{C}^{*}-\text { module }} \mathcal{M}^{\text {res }}\right|_{U \times \mathbb{C}^{*}}$ is locally free of rank less than $\operatorname{dim}_{\mathbb{C}} \overline{H^{2 *}(X)}$.

Remark 4.15. - Using Mirror symmetry, we will also prove that $\left.\mathcal{M}^{\text {res }}\right|_{U \times \mathbb{C}}$ is locally free of rank $\operatorname{dim}_{\mathbb{C}} \overline{H^{2 *}(X)}$. We refer to Remark 5.11 for a precise explanation.

Proof of Theorem 4.14. - On $z \neq 0,\left.\mathcal{M}^{\text {res }}\right|_{U \times \mathbb{C}^{*}}$ is locally free. By Nakayama's lemma, it is enough to prove the first statement.

Consider the residual Batyrev ring $B^{\text {Res }}=\Lambda\left[x_{\rho}\right] /\left(G: x_{\text {top }}\right)$ defined in Subsection 3.6. This is a $\Lambda$-algebras. Denote by $\mathcal{B}^{\text {res }}$ the associated sheaf of rings on $\mathbf{V}=\operatorname{Spec} \Lambda$. When restricted 
to the neighbourhood $\bar{U}$ of the large radius limit, $\mathcal{B}^{\text {res }}$ is locally free of rank $\operatorname{dim}_{\mathbb{C}} \overline{H^{2 *}(X)}$ by Proposition 3.40. Then, Lemma 4.16 below shows that the sheaf $\mathcal{M}^{\text {res }} / z \mathcal{M}^{\text {res }}$ is a sheaf of commutative rings defined over $U$ which is isomorphic to the $\left.\mathcal{B}^{\text {res }}\right|_{U}$.

In the following, we use notations of the proof of Theorem 4.10. The set $\mathcal{P}$ of primitive classes is defined in Notation 3.19. Let $\mathbb{S}:=\left\langle\square_{d}^{\prime}, d \in \mathcal{P}\right\rangle$ be the "square" ideal in $\mathbb{A}_{2}:=$ $\mathbb{C}\left[q^{ \pm}, z\right]\left\langle z \delta_{\lambda}\right\rangle$. Put $\widehat{c}_{\text {top }}^{\lambda}:=\prod_{\rho \in \Delta(1)}^{\text {v.b. }} z \delta_{\lambda_{\rho}} \in \mathbb{A}_{2}$. Do not confuse it with $\widehat{c}_{\text {top }}:=\prod_{i=1}^{k} \widehat{\mathcal{L}}_{i} \in \mathbb{D}^{\prime}$ written in terms of $z \delta_{q}$ 's operators. Recall that

$$
\mathrm{QSR}:=\left\langle R_{d}:=x^{d^{+}}-q^{d} x^{d^{-}}, d \in \mathrm{NE}(Y)_{\mathbb{Z}}\right\rangle
$$

We put :

$$
\begin{aligned}
& \operatorname{Quot}\left(\widehat{c}_{\mathrm{top}}^{\lambda}, \mathbb{S}\right):=\left\langle P \in \mathbb{A}_{2}, \widehat{c}_{\text {top }}^{\lambda} P \in \mathbb{S}\right\rangle \\
& \left(\text { QSR }: x_{\text {top }}\right):=\left\{P \in \mathbb{C}\left[q^{ \pm}\right]\left[x_{\rho}\right], P . x_{\text {top }} \in \text { QSR }\right\}
\end{aligned}
$$

We introduced $x_{\text {top }}:=\prod_{\rho \in \Delta(1)}$ v.b. $x_{\rho}$ in the beginning of Subsection 3.6. Notice that (QSR $: x_{\text {top }}$ ) is the usual quotient ideal of commutative algebra. The set $\left\{P \in \mathbb{C}\left[q^{ \pm}\right]\left[x_{\rho}\right], x_{\text {top }} P \in\right.$ QSR $\}$ is an ideal. However, in the non commutative ring $\mathbb{A}_{2}$, the set $\left\{P \in \mathbb{A}_{2}, \widehat{c}_{\text {top }}^{\lambda} P \in \mathbb{S}\right\}$ is not an ideal anymore (cf. footnote 3$)$.

Lemma 4.16. - $\quad$ 1. There exists an isomorphism of commutative $\mathbb{C}\left[q^{ \pm}\right]$-algebras :

$$
\begin{aligned}
h: \mathbb{A}_{2} /\left(\operatorname{Quot}\left(\widehat{c}_{\mathrm{top}}^{\lambda}, \mathbb{G}_{2}\right)+\langle z\rangle\right) & \longrightarrow \mathbb{C}\left[q^{ \pm}\right]\left[x_{\rho}\right] /\left(\mathrm{QSR}: x_{\mathrm{top}}\right) \\
z & \longmapsto 0 \\
z \delta_{\lambda_{\rho}} & \longmapsto \begin{cases}x_{\rho} & \text { if } \rho \in \Delta_{(1)}^{\text {base }} \\
-x_{\rho} & \text { if } \rho \in \Delta_{(1)}^{\text {v.b. }}\end{cases}
\end{aligned}
$$

2. We pass to the quotient by linear ideals i.e.,

- on the left side, we quotient by $\left\langle\mathcal{Z}_{u}:=\sum_{\rho \in \Delta(1)}\left\langle u, v_{\rho}\right\rangle z \delta_{\rho}\right.$, for $\left.u \in M^{\prime},\right\rangle$

- on the right side, we quotient by $\left\langle Z_{u}:=\sum_{\rho \in \Delta(1)}\left\langle u, v_{\rho}\right\rangle x_{\rho}\right.$, for $u \in M^{\prime}$. to obtain an isomorphism:

$$
\mathbb{M}^{\mathrm{res}} / z \mathbb{M}^{\mathrm{res}} \longrightarrow B^{\mathrm{res}}
$$

between the residual GKZ-module restricted to $z=0$ and the residual Batyrev ring.

Proof. - The second statement follows easily from the first one.

The morphism $h$ is well defined since $h\left(\square_{d}\right)=R_{d}, h\left(\widehat{c}_{\text {top }}^{\lambda}\right)=x_{\text {top }}$ and that setting $z=0$ makes the algebra $\mathbb{A}_{2}$ becomes commutative. It is surjective by construction. We construct its inverse morphism. Consider the isomorphism of commutative $\mathbb{C}\left[q^{ \pm}\right]$-algebras, where $P$ is sent on $\widehat{P}$ :

$$
\begin{aligned}
& \mathbb{C}\left[q^{ \pm}\right]\left[x_{\rho}\right] \longrightarrow \mathbb{A}_{2} /\langle z\rangle \\
& x_{\rho} \longmapsto \widehat{x}_{\rho}=\overline{z \delta_{\lambda_{\rho}}}
\end{aligned}
$$

Where the overline notation means its image in the quotient $\mathbb{A}_{2} /\langle z\rangle$. Recall that for $d \in$ $H_{2}(Y, \mathbb{Z})$, we denote $R_{d}:=x^{d^{+}}-q^{d} x^{d^{-}}$. We have $\widehat{R}_{d}=\overline{\square_{d}^{\prime}}$, and any element of QSR maps to $\overline{\mathbb{G}}_{2}$ (see after $(4.12)$ for the definition of $\mathbb{G}_{2}$ ).

Let $P$ be in (QSR : $\left.x_{\text {top }}\right)$, we show that $\widehat{P}$ belongs to $\overline{\operatorname{Quot}\left(\widehat{c}_{\text {top }}^{\lambda}, \mathbb{G}_{2}\right)}$. Then the morphism defined in (4.17) will induce the inverse of $h$.

There exists a set of polynomials $\left\{A_{d}, d \in \mathcal{P}\right\}$ such that:

$$
x_{\mathrm{top}} P=\sum_{d \in \mathcal{P}} A_{d}(q, x) R_{d}(q, x)
$$


For such an expression above consider the biggest (using the order $\prec_{\varphi}$ see $\S 3.4$.a), leading monomial that appears in $A_{d}(q, x) R_{d}(q, x)$ for $d \in \mathcal{P}$. Among all the expression of (4.18), denote by $m(P)$ the smallest of these leading monomials that is :

$$
m(P):=\min _{\substack{\left(A_{d}\right) d \in \mathcal{P} \\ P=\sum A_{d} R_{d}}} \max \left\{\operatorname{Lm}\left(A_{d} R_{d}\right), d \in \mathcal{P}\right\}
$$

where $\operatorname{Lm}(S)$ is the leading monomial of a polynomial $S$. Notice that $m(P)$ could be different than $\operatorname{Lm}(P)$.

As the function $\varphi$ used to define the order $\prec_{\varphi}$ is associated to an ample divisor we can assume -up to a change of ample divisor- that $\varphi\left(x_{\rho}\right)<0$ for any $\rho \in \Delta(1)$. In particular, the set of monomial smaller than a fixed monomial $m$ is finite, and possess a smaller element, namely the monomial 1 . We will prove by induction on the monomial $m$ :

$$
H(m)=" \forall P \in\left(x_{\text {top }}: \text { QSR }\right), m(P) \prec_{\varphi} m \Longrightarrow \widehat{P} \in \overline{\operatorname{Quot}\left(\widehat{c}_{\text {top }}^{\lambda}, \mathbb{S}\right)} "
$$

If $m=1$, then $x_{\text {top }} P$ is a constant polynomials, which is only possible if $P=0$. Then we have $\widehat{P} \in \operatorname{Quot}\left(\widehat{c}_{\text {top }}^{\lambda}, \mathbb{S}\right)$.

Put $m:=m(P)$ and consider a minimal expression for (4.18) i.e., polynomials $A_{d}$ for $d \in \mathcal{P}$ such that :

$$
x_{\text {top }} P=\sum_{d \in \mathcal{P}} A_{d} R_{d} \text { with } \max \left\{\operatorname{Lm}\left(A_{d} R_{d}\right), d \in \mathcal{P}\right\}=m .
$$

Let $\mathcal{P}^{*}$ be the subset of $\mathcal{P}$ such that $\operatorname{Lm}\left(A_{d} R_{d}\right)=m$. This set is not empty by assumption. If $d$ is in $\mathcal{P}^{*}, A_{d}$ can be written :

$$
A_{d}=\alpha_{d} n_{d}+B_{d}
$$

where $\alpha_{d} \in \mathbb{C}\left[q^{ \pm}\right], n_{d}=\operatorname{Lm}\left(A_{d}\right), \operatorname{Lm}\left(A_{d} R_{d}\right)=\operatorname{Lm}\left(A_{d}\right) \cdot \operatorname{Lm}\left(R_{d}\right)=n_{d} \cdot x^{d^{+}}=m$ and $\operatorname{Lm}\left(B_{d} R_{d}\right) \prec_{\varphi} m$.

If $d \in \mathcal{P} \backslash \mathcal{P}^{*}$ we simply set $B_{d}:=A_{d}$, so that :

$$
x_{\mathrm{top}} P=\sum_{d \in \mathcal{P}^{*}} \alpha_{d} n_{d} R_{d}+\sum_{d \in \mathcal{P}} B_{d} R_{d}
$$

with $\operatorname{Lm}\left(B_{d} R_{d}\right) \prec_{\varphi} m$ for any $d \in \mathcal{P}$.

Consider two cases :

Case $1: x_{\text {top }}$ divides $m$. Then, for any $d \in \mathcal{P}^{*}, x_{\text {top }}$ divides $n_{d} x^{d^{+}}$. Since for any $\rho \in \Delta_{(1)}^{\text {v.b. }}$, the variable $x_{\rho}$ does not appear in $x^{d^{+}}$(because the $\mathcal{L}_{i}$ 's are ample and $\mathcal{P} \subset N E(Y)$ ), then $x_{\text {top }}$ divides $n_{d}$ for any $d \in \mathcal{P}^{*}$. Set $n_{d}=x_{\text {top }} n_{d}^{\prime}$. We find :

$$
x_{\text {top }} P=x_{\text {top }}\left(\sum_{d \in \mathcal{P}^{*}} \alpha_{d} n_{d}^{\prime} R_{d}\right)+\sum_{d \in \mathcal{P}} B_{d} R_{d} .
$$

and the polynomial $S=P-\sum_{d \in \mathcal{P}^{*}} n_{d}^{\prime} R_{d}$ is in (QSR : $x_{\mathrm{top}}$ ) and satisfy $m(S) \prec_{\varphi} m(P)$. By induction, the operator $\widehat{S} \in \mathbb{A}_{2} /\langle z\rangle$ is in $\operatorname{Quot}\left(\widehat{c}_{\mathrm{top}}^{\lambda}, \mathbb{S}\right)$. Moreover, the operator

$$
\widehat{P}=\widehat{S}+\sum_{d \in \mathcal{P}^{*}} \alpha_{d} \widehat{n}_{d}^{\prime} \widehat{R}_{d}=\widehat{S}+\sum_{d \in \mathcal{P}^{*}} \alpha_{d} \widehat{n}_{d}^{\prime} \overline{\square_{d}^{\prime}}
$$

is also in $\overline{\operatorname{Quot}\left(\widehat{c}_{\mathrm{top}}^{\lambda}, \mathbb{S}\right)}$.

Case $2: x_{\text {top }}$ does not divide $m$. Since $\left(\sum_{d \in \mathcal{P}^{*}} \alpha_{d} n_{d} R_{d}\right)+\sum_{d \in \mathcal{P}} B_{d} R_{d}=x_{\text {top }} P$, the coefficient of $m$ in the sum $\left(\sum_{d \in \mathcal{P}^{*}} \alpha_{d} n_{d} R_{d}\right)$ must be zero. This coefficient is exactly $\sum_{d} \alpha_{d}$. Fix a class $c$ in $\mathcal{P}^{*}$; then $\alpha_{c}=-\sum_{d \in \mathcal{P}^{*} \backslash\{c\}} \alpha_{d}$, and we have :

$$
\sum_{d \in \mathcal{P}^{*}} \alpha_{d} n_{d} R_{d}=\alpha_{c} n_{c} R_{c}+\sum_{d \in \mathcal{P}^{*} \backslash\{c\}} \alpha_{d} n_{d} R_{d}=\sum_{d \in \mathcal{P}^{*} \backslash\{c\}} \alpha_{d}\left(n_{d} R_{d}-n_{c} R_{c}\right)
$$


But we have $n_{d} R_{d}-n_{c} R_{c}=n_{d}\left(x^{d^{+}}-q^{d} x^{d^{-}}\right)-n_{c}\left(x^{c^{+}}-q^{d} x^{c^{-}}\right)$and $n_{d} x^{d^{+}}=n_{c} x^{c^{+}}=m$, which gives

$$
n_{d} R_{d}-n_{c} R_{c}=-n_{d} q^{d} x^{d^{-}}+n_{c} q^{d} x^{c^{-}} .
$$

Moreover, $x_{\text {top }}$ divides any $x^{d^{-}}$for any $d \in \mathcal{P}$ (because the $\mathcal{L}_{i}$ 's are ample and $\mathcal{P} \subset$ $N E(Y))$. Denote by $\epsilon=\left(\epsilon_{\rho}\right)_{\rho \in \Delta(1)}$ the multi-index that equals to 0 for $\rho \in \Delta_{(1)}^{\text {base }}$ and 1 for $\rho \in \Delta^{\text {v.b. }}(1)$. We have $x^{d^{-}}=x_{\text {top }} x^{d^{-}-\epsilon}$. This gives, in (4.19) :

$$
n_{d} R_{d}-n_{c} R_{c}=x_{\mathrm{top}}\left(n_{c} q^{d} x^{c^{-}-\epsilon}-n_{d} q^{d} x^{d^{-}-\epsilon}\right) .
$$

For $d \in \mathcal{P}^{*} \backslash\{c\}$ set $C_{d}:=n_{c} q^{d} x^{c^{-}-\epsilon}-n_{d} q^{d} x^{d^{-}-\epsilon}$. We get :

$$
x_{\mathrm{top}} P=x_{\mathrm{top}}\left(\sum_{d \in \mathcal{P}^{*} \backslash\{c\}} \alpha_{d} C_{d}\right)+\sum_{d \in \mathcal{P}} B_{d} R_{d} .
$$

The polynomial $S:=P-\left(\sum_{d \in \mathcal{P}^{*} \backslash\{c\}} \alpha_{d} C_{d}\right)$ is in (QSR : $\left.x_{\text {top }}\right)$ and satisfies $m(S) \prec_{\varphi} m(P)$. By induction, the operator $\widehat{S}$ is in $\overline{\operatorname{Quot}\left(\widehat{c}_{\text {top }}^{\lambda}, \mathbb{S}\right)}$. Moreover, for any $d \in \mathcal{P}^{*} \backslash\{c\}$, the equality $x_{\mathrm{top}} C_{d}=n_{d} R_{d}-n_{c} R_{c}$ gives in $\mathbb{A}_{2} /\langle z\rangle$ :

$$
\widehat{c}_{\text {top }}^{\lambda} \widehat{C}_{d}=\widehat{n}_{d} \overline{\square_{d}^{\prime}}-\widehat{n}_{c} \bar{\square}_{c}^{\prime} .
$$

We deduce that $\widehat{C}_{d}$ is in $\overline{\operatorname{Quot}\left(\widehat{c}_{\text {top }}^{\lambda}, \mathbb{S}\right)}$. Finally, we have

$$
\widehat{P}=\left(\sum_{d \in \mathcal{P}^{*} \backslash\{c\}} \alpha_{d} \widehat{C}_{d}\right)+\widehat{S} \in \overline{\operatorname{Quot}\left(\widehat{c}_{\text {top }} \backslash \mathbb{S}\right)} .
$$

\section{Isomorphisms between quantum $\mathcal{D}$-modules and GKZ systems via mirror symmetry}

5.1. Recalls on Mirror symmetry. - We start by some recalls on mirror symmetry in the framework of Givental. Here, we suppose that $X$ is a smooth toric projective variety endowed with $k$ globally generated line bundles $\mathcal{L}_{1}, \ldots, \mathcal{L}_{k}$ such that $\left(\omega_{X} \otimes \mathcal{L}_{1} \otimes \cdots \otimes \mathcal{L}_{k}\right)^{\vee}$ is nef. We put $\mathcal{E}=\oplus_{i=1}^{k} \mathcal{L}_{i}$.

We introduce a cohomological multi-valued function which will play a central role in mirror symmetry. Recall that $t_{0}$ is the coordinate on $H^{0}(X)$. In the definition below, we use the notation $\mathcal{E}_{0,1, d}(1)$ for the vector bundle on $X_{0,1, d}$ defined in Subsection 2.1.a.

Definition 5.1. - We define local section $J^{\text {tw }}$ of $F=H^{2 *}(X) \times\left(H^{0}(X) \times \bar{V} \times \mathbb{C}\right) \rightarrow$ $\left(H^{0}(X) \times \bar{V} \times \mathbb{C}\right)$ by :

$$
J^{\mathrm{tw}}\left(t_{0}, q, z\right):=e^{t_{0} / z} q^{T / z}\left(1+z^{-1} \sum_{\substack{d \in H_{2}(X, \mathbb{Z}) \\ d \neq 0}} q^{d} e_{1 *}\left(\frac{c_{\mathrm{top}}\left(\mathcal{E}_{0,1, d}(1)\right)}{z-\psi} \cap\left[X_{0,1, d}\right]^{\mathrm{vir}}\right)\right)
$$

where $t_{0}$ is in $H^{0}(X), q$ is in $\bar{V}, z$ is in $\mathbb{C}, \psi$ is defined before Definition 2.5 and $q^{T / z}=$ $q_{1}^{T_{1} / z} \ldots q_{r}^{T_{r} / z}:=e^{z^{-1} \sum_{a=1}^{r} T_{a} \log \left(q_{a}\right)}$ as in the definition of the function $L^{\mathrm{tw}}$ (Formula 2.16).

The relation between this function $J^{\mathrm{tw}}$ and $L^{\mathrm{tw}}$ is given by the following proposition. 
Proposition 5.2. - We have

$$
\begin{aligned}
c_{\text {top }}(\mathcal{E}) J^{\mathrm{tw}}\left(t_{0}, q, z\right) & =e^{t_{0} / z} q^{T / z} c_{\mathrm{top}}(\mathcal{E})\left(1+O\left(z^{-2}\right)\right) \\
& =e^{t_{0} / z} q^{T / z}\left(c_{\mathrm{top}}(\mathcal{E})+z^{-1} \sum_{\substack{a=0 \\
s-1}} q_{\substack{d \in H_{2}(X, \mathbb{Z}) \\
d \neq 0}}\left\langle\widehat{\left.\left.\frac{T_{a} \widehat{c_{\mathrm{top}}}(\mathcal{E})}{z-\psi}\right\rangle_{0,1, d} T^{a}\right)}\right.\right. \\
& =\sum_{a=0}^{s-1} S\left(L^{\mathrm{tw}}\left(t_{0}, q, z\right) T_{a}, \mathbf{1}\right) T^{a} \\
& =c_{\mathrm{top}}(\mathcal{E})\left(L^{\mathrm{tw}}\left(t_{0}, q, z\right)\right)^{-1} \mathbf{1}
\end{aligned}
$$

Proof. - The first equality follows from the definition of $J^{\mathrm{tw}}\left(t_{0}, q, z\right)$.

By definition of twisted Gromov-Witten invariant and projection formula, we have :

$$
\begin{aligned}
\left\langle\frac{\widehat{T_{a} c_{\mathrm{top}}(\mathcal{E})}}{z-\psi}\right\rangle_{0,1, d} & =\int_{\left[X_{0,1, d}\right]^{\mathrm{vir}}} e_{1}^{*}\left(T_{a} \cup c_{\mathrm{top}}(\mathcal{E})\right) \cup \frac{c_{\mathrm{top}}\left(\mathcal{E}_{0,1, d}(1)\right)}{z-\psi} \\
& =\int_{X} T_{a} \cup c_{\mathrm{top}}(\mathcal{E}) \cup e_{1 *}\left(\frac{c_{\mathrm{top}}\left(\mathcal{E}_{0,1, d}(1)\right)}{z-\psi} \cap\left[X_{0,1, d}\right]^{\mathrm{vir}}\right)
\end{aligned}
$$

We deduce the second equality from

$$
\sum_{a=0}^{s-1}\left\langle\frac{\widetilde{T_{a} c_{\mathrm{top}}(\mathcal{E})}}{z-\psi}\right\rangle_{0,1, d} T^{a}=c_{\mathrm{top}}(\mathcal{E}) e_{1 *}\left(\frac{c_{\mathrm{top}}\left(\mathcal{E}_{0,1, d}(1)\right)}{z-\psi} \cap\left[X_{0,1, d}\right]^{\mathrm{vir}}\right) .
$$

Let us show the third equality. Using Proposition A.2 (twisted $S_{n}$ invariance) and Proposition A.4 (twisted string equation) we deduce that for $d \neq 0$ in $H_{2}(X, \mathbb{Z})$,

$$
\left\langle\frac{T_{a}}{z-\psi}, \widetilde{c_{\mathrm{top}}(\mathcal{E})}\right\rangle_{0,2, d}=z^{-1}\left\langle\widehat{\frac{T_{a} c_{\mathrm{top}}(\mathcal{E})}{z-\psi}}\right\rangle_{0,1, d}
$$

Using Formula (2.16) for $L^{\mathrm{tw}}(q, z)$, we have

$$
\begin{aligned}
\sum_{a=0}^{s-1} S & \left(L^{\mathrm{tw}}\left(t_{0}, q, z\right) T_{a}, \mathbf{1}\right) T^{a} \\
& =e^{t_{0} / z} \sum_{a=0}^{s-1}\left(q^{T / z} T_{a}, \mathbf{1}\right)^{\mathrm{tw}} T^{a} \\
& +e^{t_{0} / z} \sum_{a=0}^{s-1} \sum_{b=0}^{s-1} \sum_{\substack{d \in H_{2}(X, \mathbb{Z}) \\
d \neq 0}} q^{d}\left\langle\frac{q^{T / z} T_{a}}{z-\psi}, \widetilde{T}_{b}\right\rangle_{0,2, d}\left(T^{b}, \mathbf{1}\right)^{\mathrm{tw}} T^{a} \\
& =e^{t_{0} / z}\left(q^{T / z} c_{\mathrm{top}}(\mathcal{E})+\sum_{a=0}^{s-1} \sum_{\substack{d \in H_{2}(X, \mathbb{Z}) \\
d \neq 0}} q^{d}\left\langle\frac{q^{T / z} T_{a}}{z-\psi}, \widetilde{c_{\mathrm{top}}(\mathcal{E})}\right\rangle_{0,2, d} T^{a}\right)
\end{aligned}
$$

As the expression above does not depend on the choice of a basis. Let us choose the basis $\left(q^{-T / z} T_{a}\right)_{a \in\{0, \ldots, s-1\}}$ whose dual basis is $\left(q^{T / z} T^{a}\right)_{a \in\{0, \ldots, s-1\}}$. Then we get 


$$
\begin{aligned}
& \sum_{a=0}^{s-1}\left(e^{t_{0} / z} L^{\mathrm{tw}}(q,-z) T_{a}, \mathbf{1}\right)^{\mathrm{tw}} T^{a} \\
& =e^{t_{0} / z} q^{T / z}\left(c_{\mathrm{top}}(\mathcal{E})+\sum_{\substack { a=0 \\
\begin{subarray}{c}{d \in H_{2}(X, \mathbb{Z}) \\
d \neq 0{ a = 0 \\
\begin{subarray} { c } { d \in H _ { 2 } ( X , \mathbb { Z } ) \\
d \neq 0 } }\end{subarray}} q^{d}\left(\left\langle\frac{T_{a}}{z-\psi} \widehat{c_{\mathrm{top}}(\mathcal{E})}\right\rangle_{0,2, d} T^{a}\right)\right.
\end{aligned}
$$

Then you apply (5.3) and we get the desired equality.

Show the last equality. From Proposition 2.20, we deduce that

$$
S\left(L^{\mathrm{tw}}\left(t_{0}, q, z\right) T_{a}, L^{\mathrm{tw}}\left(t_{0}, q, z\right) \mathbf{1}\right)=S\left(T_{a}, \mathbf{1}\right) .
$$

Recall that $(\cdot, \cdot)$ is the Poincaré Duality on $X$. We deduce that

$$
\begin{aligned}
\sum_{a=0}^{s-1} S\left(L^{\mathrm{tw}}\left(t_{0}, q, z\right) T_{a}, \mathbf{1}\right) T^{a} & =\sum_{a=0}^{s-1}\left(T_{a}, c_{\mathrm{top}}(\mathcal{E})\left(L^{\mathrm{tw}}\left(t_{0}, q, z\right)\right)^{-1} \mathbf{1}\right) T^{a} \\
& =c_{\mathrm{top}}(\mathcal{E})\left(L^{\mathrm{tw}}\left(t_{0}, q, z\right)\right)^{-1} \mathbf{1} .
\end{aligned}
$$

We deduce a relation with $\bar{L}$.

Corollary 5.4. - We have, in the reduced cohomology ring $H^{2 *}(X) / \operatorname{ker} m_{c_{t o p}}$ :

$$
\overline{J^{\mathrm{tw}}}\left(t_{0}, q, z\right)=\left(\bar{L}\left(t_{0}, q, z\right)\right)^{-1} \overline{\mathbf{1}} .
$$

Proof. - The last equality of Proposition 5.2 implies that $\overline{J^{\mathrm{tw}}\left(t_{0}, q, z\right)}=\overline{\left(L^{\mathrm{tw}}\left(t_{0}, q, z\right)\right)^{-1} \mathbf{1}}$ which is $\left(\bar{L}\left(t_{0}, q, z\right)\right)^{-1} \overline{\mathbf{1}}$ by definition of $\bar{L}$ (cf. Formula (2.32)).

Recall that to a ray $\theta \in \Sigma(1)$, we associate a toric divisor denoted by $D_{\theta}$. For any classes $d \in H_{2}(X, \mathbb{Z})$, put

$$
d_{\theta}:=\int_{d} D_{\theta} \text { and } d_{L_{i}}:=\int_{d} L_{i}=\int_{d} c_{1}\left(\mathcal{L}_{i}\right)
$$

We define a cohomological multi-valued function by

$$
I(q, z):=q^{T / z} \sum_{d \in H_{2}(X, \mathbb{Z})} q^{d} A_{d}(z)
$$

where

$$
\begin{aligned}
A_{d}(z) & :=\prod_{i=1}^{k} \frac{\prod_{m=-\infty}^{d_{L_{i}}}\left(\left[L_{i}\right]+m z\right)}{\prod_{m=-\infty}^{0}\left(\left[L_{i}\right]+m z\right)} \prod_{\theta \in \Sigma(1)} \frac{\prod_{m=-\infty}^{0}\left(\left[D_{\theta}\right]+m z\right)}{\prod_{m=-\infty}^{d_{\theta}}\left(\left[D_{\theta}\right]+m z\right)} \\
q^{T / z} & :=e^{z^{-1} \sum_{a=1}^{r} T_{a} \log \left(q_{a}\right)} .
\end{aligned}
$$

The mirror theorem of Givental ( $c f$. [Giv98, Theorem 0.1] and [CG07, Corrolary 5]. See also [CK99, Theorem 11.2.16] ) tells us the following.

Theorem 5.6. - [CG07, Corrolary 5] Let $X$ be a smooth toric projective variety with $k$ globally generated line bundles $\mathcal{L}_{1}, \ldots, \mathcal{L}_{k}$ such that $\left(\omega_{X} \otimes \mathcal{L}_{1} \otimes \cdots \otimes \mathcal{L}_{k}\right)^{\vee}$ is nef. There exists a neighborhood $\bar{W}$ of the large radius limit $q=0$ in $\mathbf{V}$, defining an open set $W:=\bar{W} \cap \mathbf{T}$ in $\mathbf{T}$ (cf. Notation 2.13) and there exists a single-valued map

$$
\text { Mir : } W \subset \mathbf{T} \rightarrow H^{0}(X) \oplus V \subset H^{0}(X) \oplus \mathbf{T}
$$


such that

$$
\operatorname{Mir}(q)=(0, q)+O(q) \text { and } J^{\mathrm{tw}}(\operatorname{Mir}(q), z)=\frac{I(q, z)}{F(q)}
$$

where $F(q)$ is an invertible function which is the first term in the development of the function $I$ in the power of $z^{-1}$ that is

$$
I(q, z):=F(q) \mathbf{1}+O\left(z^{-1}\right) .
$$

Proof. - Most of the statements are proved in Corrolary 5 of [CG07]. The single-valued of the map Mir is proved in the section 4.1 of [Iri09]. The two things that are not proved are the statements about the existence of the neighborhood $W$ and the asymptotic of Mir. To compute the mirror map, we develop the function $I$ in the power of $z^{-1}$, we have

$$
I(q, z)=F(q) \mathbf{1}+z^{-1} \sum_{a=0}^{r} G_{a}(q) T_{a}+O\left(z^{-2}\right) .
$$

where $F(0)=1$. Then we have :

$$
\operatorname{Mir}(q):=\Psi \circ \pi\left(F(q)^{-1} \sum_{a=0}^{r} G_{a}(q) T_{a}\right)
$$

where $\pi$ the quotient map $H^{0}(X) \oplus H^{2}(X) \rightarrow H^{0}(X) \oplus H^{2}(X) / H^{2}(X, \mathbb{Z})$ and $\Psi$ is an isomorphism between $H^{2}(X) / H^{2}(X, \mathbb{Z})$ and $\mathbf{T}$ (Formula $(2.10)$ ) We will prove that

$$
F(q)^{-1} \sum_{a=0}^{r} G_{a}(q) T_{a}=\sum_{a=1}^{r} T_{a} \log \left(q_{a}\right)+O(q)
$$

This will imply both statements on the map Mir.

To prove equality (5.7), we need to develop the function $I$ with respect to $z^{-1}$. Denote by $\mathrm{NE}(X)_{\mathbb{Z}}$ the (integral) Mori cone of $X$. From [CK99, Proof of Proposition 5.5.4 p.100] we deduce that the terms in the definition of the $I$ function (see (5.5)) vanish when $d \notin \mathrm{NE}(X)_{\mathbb{Z}}$, that is we have :

$$
I(q, z)=q^{T / z} \sum_{d \in \operatorname{NE}(X)_{\mathbb{Z}}} q^{d} A_{d}(z) .
$$

As for any $i \in\{1, \ldots, k\}$ the divisor $L_{i}$ is nef ${ }^{(5)}$, we deduce that $d_{L_{i}} \geq 0$ for $d \in \mathrm{NE}(X)_{\mathbb{Z}}$ that is

$$
A_{d}(z)=\prod_{i=1}^{k} \prod_{m=1}^{d_{L_{i}}}\left(\left[L_{i}\right]+m z\right) \prod_{\theta \in \Sigma(1)} \frac{\prod_{m=-\infty}^{0}\left(\left[D_{\theta}\right]+m z\right)}{\prod_{m=-\infty}^{d_{\theta}}\left(\left[D_{\theta}\right]+m z\right)}
$$

We develop the cohomological function $I(q, z)$ with respect to $z^{-1}$ to the order 1 . For any $\theta \in \Sigma(1)$, put $\varepsilon\left(d_{\theta}\right)=1$ if $d_{\theta}<0$ and 0 otherwise. We find that $I(q, z)$ is equal to

$$
\begin{aligned}
& \sum_{d \in \mathrm{NE}(X)_{\mathbb{Z}}} q^{d} z^{d_{\left(K_{X}+\sum_{i} L_{i}\right)}-\sum_{\theta \in \Sigma(1)} \varepsilon\left(d_{\theta}\right)}(-1)^{\sum_{\theta \in \Sigma(1)} d_{\theta}^{-}}\left(\prod_{i=1}^{k} d_{L_{i}} !\right) \prod_{\theta \in \Sigma(1)}\left(-\left[D_{\theta}\right]\right)^{\varepsilon\left(d_{\theta}\right)} \frac{\left(d_{\theta}^{-}-\varepsilon\left(d_{\theta}\right)\right) !}{d_{\theta}^{+} !} \\
& {\left[\mathbf{1}+z^{-1}\left(\sum_{a=1}^{r} T_{a} \log \left(q_{a}\right)+\sum_{i=1}^{k}\left[L_{i}\right] \sum_{m=1}^{d_{L_{i}}} \frac{1}{m}-\sum_{\theta \in \Sigma(1)}\left[D_{\theta}\right] \sum_{m=1}^{\left|d_{\theta}\right|-\varepsilon\left(d_{\theta}\right)} \frac{1}{m}\right)+O\left(z^{-2}\right)\right]}
\end{aligned}
$$

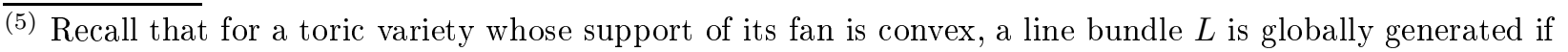
and only if $L$ is nef ( $c f$. [Mus, Proposition 7 p.22 chapter VI]). 
We decompose the Mori cone in four disjoint subsets :

$$
\begin{aligned}
& A:=\left\{d \in \mathrm{NE}(X)_{\mathbb{Z}} \mid d_{\left(K_{X}+\sum_{i} L_{i}\right)}=0 \text { and } d_{\theta} \geq 0, \forall \theta \in \Sigma(1)\right\} \\
& B:=\left\{d \in \mathrm{NE}(X)_{\mathbb{Z}} \mid d_{\left(K_{X}+\sum_{i} L_{i}\right)}=0 \text { and } \exists ! \theta_{0} \in \Sigma(1), \varepsilon\left(d_{\theta_{0}}\right)=1\right\} \\
& C:=\left\{d \in \mathrm{NE}(X)_{\mathbb{Z}} \mid d_{\left(K_{X}+\sum_{i} L_{i}\right)}=-1 \text { and } \varepsilon\left(d_{\theta}\right)=0, \forall \theta \in \Sigma(1)\right\} \\
& D:=\operatorname{NE}(X)_{\mathbb{Z}} \backslash\{A \coprod B \coprod C\} .
\end{aligned}
$$

As $-K_{X}-L_{1}-\cdots-L_{k}$ is nef, for any $d \in \mathrm{NE}(X)_{\mathbb{Z}}$ we have $d_{\left(K_{X}+L_{1}+\cdots+L_{k}\right)} \leq 0$. So the first term in the Taylor expansion is the constant term which appears only for $d \in A$. This term takes value in $H^{0}(X)$ i.e., it is $F(q) 1$ with $F$ the following scalar function :

$$
F(q):=\sum_{d \in A} q^{d} \frac{\prod_{i=1}^{k} d_{L_{i}} !}{\prod_{\theta \in \Sigma(1)} d_{\theta} !}
$$

Notice that this function is invertible in a neighborhood of $q=0$ because $d=0$ belongs to $A$ so that $F(q) \neq 0$ in a suitable neighborhood of $q=0$.

Compute the term in front of $z^{-1}$ :

1. from $A$, we get the following element in $H^{2}(X)$

$$
z^{-1}\left[\sum_{d \in A} q^{d} \frac{\prod_{i=1}^{k} d_{L_{i}} !}{\prod_{\theta}\left(d_{\theta}\right) !}\left(\left(\sum_{a=1}^{r} T_{a} \log q_{a}\right)+\sum_{i=1}^{k}\left[L_{i}\right] \sum_{m=1}^{d_{L_{i}}} m^{-1}-\sum_{\theta \in \Sigma(1)}\left[D_{\theta}\right] \sum_{m=1}^{d_{\theta}} \frac{1}{m}\right)\right]
$$

2. from $B$, we get

$$
z^{-1}\left[D_{\theta_{0}}\right]\left(\sum_{d \in B} q^{d}(-1)^{-d_{\theta_{0}}-1}\left(\prod_{i=1}^{k} d_{L_{i}} !\right)\left(-d_{\theta_{0}}-1\right) ! \prod_{\theta \neq \theta_{0}} \frac{1}{\left(d_{\theta}\right) !}\right) \in H^{2}(X)
$$

3. from $C$, we get

$$
z^{-1} \mathbf{1}\left(\sum_{d \in C} q^{d} \frac{\prod_{i=1}^{k} d_{L_{i}} !}{\prod_{\theta} d_{\theta} !}\right) \in H^{0}(X)
$$

Now we develop with respect to $q$ when $q$ is near 0 . As $d=0$ belongs only to subset $A$, we deduce Equality (5.7).

Remark 5.9. - If we are in the most famous case of the quintic in $X:=\mathbb{P}^{4}$ that is $\mathcal{L}=$ $\mathcal{O}(5)$. We have $\operatorname{NE}(X)_{\mathbb{Z}}=\mathbb{N}, \mathcal{L} \otimes \omega_{X}$ is trivial, and the toric divisor $D_{\theta}$ satisfies $\left[D_{\theta}\right]=H \in$ $H^{2}(X)$, where $H=c_{1}(\mathcal{O}(1))$. For any $d \in \mathrm{NE}\left(()_{\mathbb{Z}} X\right) \subset H_{2}(X, \mathbb{Z})$, we have $d_{\theta}=d \geq 0$ and $d_{L}=5 d$. The subset $A$ is $\mathbb{N}$ and $B, C$ are empty so that

$$
F(q)=\sum_{d \geq 0} q^{d} \frac{(5 d) !}{(d !)^{5}}
$$

and the term in front of $z^{-1}$ is

$$
\begin{aligned}
& \sum_{d \in \mathbb{N}} q^{d} \frac{(5 d) !}{(d !)^{5}}\left(H \log q+5 H \sum_{m=1}^{5 d} \frac{1}{m}-5 H \sum_{m=1}^{d} \frac{1}{m}\right) \\
= & H\left[F(q) \log q+5\left(\sum_{d \geq 1} q^{d} \frac{(5 d) !}{(d !)^{5}} \sum_{m=d+1}^{5 d} \frac{1}{m}\right)\right] .
\end{aligned}
$$


5.2. Isomorphism's theorems. - We can now state our main theorem. Recall from Section 2 that we defined $\operatorname{QDM}(X, \mathcal{E}):=\left(F, \nabla, S, F_{\mathbb{Z}}\right)$ and $\overline{\operatorname{QDM}}(X, \mathcal{E}):=\left(\bar{F}, \bar{\nabla}, \bar{S}, \bar{F}_{\mathbb{Z}}\right)$ where $F$ and $\bar{F}$ are bundle over $V \times \mathbb{C}$. We denote by $\mathcal{F}($ resp. $\overline{\mathcal{F}})$ the sheaf of sections of $F$ $($ resp. $\bar{F})$.

Recall that $W$ is a neighborhood of the large radius limit $q=0$ in $\mathbf{T} \subset \mathbf{V}$. In Theorem 5.6, we defined a map

$$
\begin{aligned}
\operatorname{Mir} \times \operatorname{id}: W \times \mathbb{C} & \rightarrow H^{0}(X) \times V \times \mathbb{C} \\
(q, z) & \mapsto(\operatorname{Mir}(q), z)
\end{aligned}
$$

Recall from Theorem 4.10, the sheaf $\left.\mathcal{M}\right|_{U \times \mathbb{C}}$ is a vector bundle of rank $\operatorname{dim}_{\mathbb{C}} \overline{H^{2 *}(X)}$ with an integrable connection. Notice that for the sheaf $\mathcal{M}^{\text {res }}$ we only have the result of Theorem 4.14 that we do not have a priori the local freeness over $U \times \mathbb{C}$. The local freeness for $\mathcal{M}^{\text {res }}$ will follow from Theorem 5.10 below (see Remark 5.11). Recall that $\mathcal{M}$ is defined (see Definition 4.4 ) as a quotient by an ideal denoted $\mathbb{G}$. Using Notation 4.1 , we put $\widehat{c}_{\text {top }}:=\prod_{i=1}^{k} \widehat{c_{1}\left(\mathcal{L}_{i}\right)}$ that is

$$
\widehat{c}_{\mathrm{top}}:=\prod_{i=1}^{k} \sum_{a=1}^{r} L_{a}^{i} z \delta_{a}
$$

where for $i \in\{1, \ldots, k\}$ and $c_{1}\left(\mathcal{L}_{i}\right):=\sum_{a=1}^{r} L_{a}^{i} T_{a}$.

Theorem 5.10. - Let $X$ be a smooth toric variety with $k$ line bundles $\mathcal{L}_{1}, \ldots, \mathcal{L}_{k}$ such that $\left(\omega_{X} \otimes \mathcal{L}_{1} \otimes \ldots \otimes \mathcal{L}_{k}\right)^{\vee}$ is nef. We put $\mathcal{E}:=\oplus_{i=1}^{k} \mathcal{L}_{i}$. For a small real number $\varepsilon$ in $\mathbb{R}_{>0}$, put

$$
W_{\varepsilon}:=\left\{\left(q_{1}, \ldots, q_{r}\right) \in W|0<| q_{a} \mid<\varepsilon\right\} .
$$

There exists $\varepsilon$ in $\mathbb{R}_{>0}$ such that

1. If the line bundles $\mathcal{L}_{1}, \ldots, \mathcal{L}_{k}$ are globally generated, then we have the following isomorphism of sheaf of $\mathcal{O}_{W_{\varepsilon} \times \mathbb{C}^{-}}$modules :

$$
\left.\mathcal{M}\right|_{W_{\varepsilon} \times \mathbb{C}} \stackrel{\sim}{\longrightarrow}(\operatorname{Mir} \times \mathrm{id})^{*}(\mathcal{F}, \nabla)
$$

where Mir is the mirror map of Givental.

2. If the line bundles $\mathcal{L}_{1}, \ldots, \mathcal{L}_{k}$ are ample, then we have the following commutative diagram

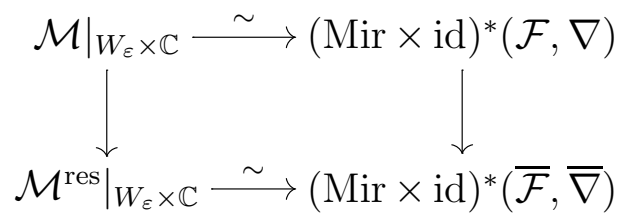

Remark 5.11. - Recall that $\left.\mathcal{M}\right|_{U \times \mathbb{C}}$ is locally free by Theorem 4.10. However, as far as is known at this point, $\left.\mathcal{M}^{\text {res }}\right|_{U \times \mathbb{C}}$ is only locally free of expected rank over $U \times\{0\}$ and locally free (of a smaller or equal rank) over $U \times \mathbb{C}^{*}$ (Theorem 4.14). Theorem 5.10, gives us local freeness of $\left.\mathcal{M}^{\text {res }}\right|_{U \times \mathbb{C}}$ (see the end of the proof).

Theorem 2.42 says that under some conditions we have $\overline{\mathrm{QDM}}(X, \mathcal{E}) \simeq \mathrm{QDM}_{\mathrm{amb}}(Z)$. So we deduce the following corollary.

Corollary 5.12. - Let $X$ be a smooth toric variety with $k$ ample line bundles $\mathcal{L}_{1}, \ldots, \mathcal{L}_{k}$ such that $\left(\omega_{X} \otimes \mathcal{L}_{1} \otimes \cdots \otimes \mathcal{L}_{k}\right)^{\vee}$ is nef. Let $Z$ be the zeros of a generic section of $\mathcal{E}:=\oplus_{i=1}^{k} \mathcal{L}_{i}$. Assume that $\operatorname{dim}_{\mathbb{C}} Z \geq 3$. We have $\left.\mathcal{M}^{\mathrm{res}}\right|_{W_{\varepsilon} \times \mathbb{C}}$ is isomorphic to $(\mathrm{Mir} \times \mathrm{id})^{*}\left(F_{\mathrm{amb}}^{Z}, \nabla_{\mathrm{amb}}^{Z}\right)$.

Remark 5.13. - This corollary answer to the question addressed in the [CK99, p.94-95 and p.101]: "What differential equations shall we add to $\mathcal{G}$ to get an isomorphism with $\mathrm{QDM}_{\mathrm{amb}}(Z) ? "$ 
To prove the Theorem 5.10, we will need some preliminary results.

We denote by $\operatorname{Mir}^{*} \nabla$ the pullback connection on the bundle $(\operatorname{Mir} \times \text { id })^{*} F \rightarrow W \times \mathbb{C}$. For an endomorphism $u$, we denote $z^{u}:=\exp (u \log z)$.

Proposition 5.14. - With the hypothesis of Theorem 5.10.

1. The morphism

$$
\begin{aligned}
\varphi:\left.\mathcal{M}\right|_{W_{\varepsilon} \times \mathbb{C}} & \longrightarrow(\operatorname{Mir} \times \mathrm{id})^{*}(\mathcal{F}, \nabla) \\
P\left(q, z, z \delta_{q}, z \delta_{z}\right) & \longmapsto L^{\mathrm{tw}}(\operatorname{Mir}(q), z) z^{-\mu} z^{c_{1}\left(\mathcal{T}_{X} \otimes \mathcal{E}^{\vee}\right)} P\left(q, z, z \delta_{q}, z \delta_{z}\right) z^{-c_{1}\left(\mathcal{T}_{X} \otimes \mathcal{E}^{\vee}\right)} z^{\mu} J^{\mathrm{tw}}(\operatorname{Mir}(q), z)
\end{aligned}
$$

is well defined.

2. The morphism above induced a well defined morphism $\varphi^{\prime}$ that make the following diagram commutative.

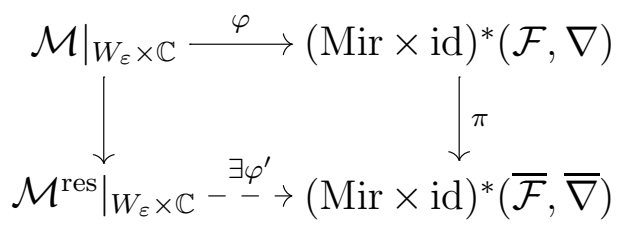

Moreover the composition morphism $\pi \circ \varphi$ sends

$$
P\left(q, z, z \delta_{q}, z \delta_{z}\right) \longmapsto P\left(q, z, z \operatorname{Mir}^{*} \nabla_{\delta_{q}}, z \operatorname{Mir}^{*} \nabla_{\delta_{z}}\right) \overline{\mathbf{1}}
$$

Remark 5.15. - We should say a word on the definition of $\varphi$ that seem quite complicated. The reason is that we want the natural expression for $\varphi^{\prime}$ which is the one above. All the problem comes from $J^{\mathrm{tw}}\left(t_{0}, q, z\right)$ is not $L^{\mathrm{tw}}\left(t_{0}, q, z\right)^{-1} \mathbf{1}$, but they are equal after cupping by $c_{\text {top }}(\mathcal{E})$ (see Proposition 5.2). Of course if we cup by $c_{\text {top }}(\mathcal{E})$ the expression above of $\varphi$, it simplifies a lot, but it will not be an isomorphism anymore.

Proof of Proposition 5.14. - From Theorem 5.6 we have that $J^{\mathrm{tw}}(\operatorname{Mir}(q), z)=I(q, z) / F(q)$. Lemma 5.16 shows that the morphism $\varphi$ is well defined.

Lemma 5.21 implies that for any $R \in \operatorname{Quot}\left(\widehat{c}_{\text {top }}, \mathbb{G}\right)$ we have

$$
\overline{R z^{-c_{1}\left(\mathcal{T}_{X} \otimes \mathcal{E}^{\vee}\right)} z^{\mu} I(q, z)}=0 .
$$

This implies that $\varphi^{\prime}$ is well defined. By Corollary 5.4, we have

$$
\overline{J^{\mathrm{tw}}}(\operatorname{Mir}(q), z)=(\bar{L}(\operatorname{Mir}(q), z))^{-1} \overline{\mathbf{1}} .
$$

We deduce that

$$
\begin{aligned}
\pi \circ \varphi\left(P\left(q, z, z \delta_{q}, \delta_{z}\right)\right) & =P\left(q, z, z \operatorname{Mir}^{*} \nabla_{\delta_{q}}, \operatorname{Mir}^{*} \nabla_{\delta_{z}}\right) \bar{L}(\operatorname{Mir}(q), z) \overline{J^{\mathrm{tw}}}(\operatorname{Mir}(q), z) \\
& =P\left(q, z, z \operatorname{Mir}^{*} \nabla_{\delta_{q}}, \operatorname{Mir}^{*} \nabla_{\delta_{z}}\right) \overline{\mathbf{1}}
\end{aligned}
$$

Lemma 5.16. - Put $q^{d}:=\prod_{a=1}^{r} q_{a}^{\int_{d} T_{a}}=\prod_{a=1}^{r} q_{a}^{d_{a}}$. For any $d \in H_{2}(X, \mathbb{Z})$, we have

$$
\begin{aligned}
\square_{d}\left(z^{-c_{1}\left(\mathcal{T}_{X} \otimes \mathcal{E}^{\vee}\right)} z^{\mu} I(q, z)\right) & =0 \\
\widehat{\mathfrak{E}}\left(z^{-c_{1}\left(\mathcal{T}_{X} \otimes \mathcal{E}^{\vee}\right)} z^{\mu} I(q, z)\right) & =0 .
\end{aligned}
$$


where

$$
\begin{aligned}
\square_{d} & :=\prod_{i=1}^{k} \prod_{\nu=1}^{d_{L_{i}}^{-}}\left(\widehat{\mathcal{L}}_{i}+\nu z\right) \prod_{\theta \in \Sigma(1)} \prod_{\nu=0}^{d_{\theta}^{+}-1}\left(\widehat{D}_{\theta}-z \nu\right) \\
& -q^{d} \prod_{i=1}^{k} \prod_{\nu=1}^{d_{L_{i}}^{+}}\left(\widehat{\mathcal{L}}_{i}+\nu z\right) \prod_{\theta \in \Sigma(1)} \prod_{\nu=0}^{d_{\theta}^{-}-1}\left(\widehat{D}_{\theta}-z \nu\right) \\
\widehat{\mathfrak{E}} & :=z \delta_{z}+c_{1}\left(\widehat{\mathcal{T}_{X} \otimes} \mathcal{E}^{\vee}\right)(\text { cf. Notation 4.1) }
\end{aligned}
$$

Proof. - In this proof, we denote $d_{\mathcal{T}_{X} \otimes \mathcal{E}^{\vee}}:=\int_{d} c_{1}\left(\mathcal{T}_{X} \otimes \mathcal{E}^{\vee}\right)$. For any $\alpha \in H^{2}(X)$, we have $[\mu, \alpha]=\alpha$. This implies that

$$
z^{\mu} \frac{\alpha}{z}=\alpha z^{\mu}
$$

From this we deduce that $z^{\mu} A_{d}(z)=z^{-d_{\mathcal{T}_{X} \otimes \mathcal{E}}} A_{d}(1)$. Using the definition (5.5) of the cohomological function $I$, we deduce that

$$
z^{-c_{1}\left(\mathcal{T}_{X} \otimes \mathcal{E}^{\vee}\right)} z^{\mu} I(q, z)=\sum_{d \in H_{2}(X, \mathbb{Z})} q^{T+d} z^{-c_{1}\left(\mathcal{T}_{X} \otimes \mathcal{E}^{\vee}\right)-d_{\mathcal{T}_{X} \otimes \mathcal{E} \vee}} A_{d}(1) .
$$

For any class $\alpha \in H^{2}(X)$, a direct computation shows that

$$
\begin{aligned}
\widehat{\alpha} q^{T+d} & =q^{T+d} z\left(\alpha+d_{\alpha}\right) \\
z \delta_{z}\left(z^{\left.-c_{1}\left(\mathcal{T}_{X} \otimes \mathcal{E}^{\vee}\right)-d_{c_{1}\left(\mathcal{T}_{X} \otimes \mathcal{E}^{\vee}\right)}\right)}\right. & =z\left(-c_{1}\left(\mathcal{T}_{X} \otimes \mathcal{E}^{\vee}\right)-d_{\mathcal{T}_{X} \otimes \mathcal{E}^{\vee}}\right) z^{-c_{1}\left(\mathcal{T}_{X} \otimes \mathcal{E}^{\vee}\right)-d_{\mathcal{T}_{X} \otimes \mathcal{E}^{\vee}}}
\end{aligned}
$$

We deduce that

$$
z \delta_{z}\left(q^{T+d} z^{-c_{1}\left(\mathcal{T}_{X} \otimes \mathcal{E}^{\vee}\right)-d_{\mathcal{T}_{X} \otimes \mathcal{E}^{\vee}}}\right)=-c_{1}\left(\widehat{\mathcal{T}_{X} \otimes} \mathcal{E}^{\vee}\right)\left(q^{T+d} z^{-c_{1}\left(\mathcal{T}_{X} \otimes \mathcal{E}^{\vee}\right)-d_{\mathcal{T}_{X} \otimes \mathcal{E}^{\vee}}}\right) .
$$

This implies the second equality of the Lemme.

Using Formula (5.19), the equality $\square_{d}\left(z^{-c_{1}\left(\mathcal{T}_{X} \otimes \mathcal{E}^{\vee}\right)} z^{\mu} I(q, z)\right)=0$ for any $d \in H_{2}(X, \mathbb{Z})$ reduced to the equality below. For any $d, d^{\prime} \in H_{2}(X, \mathbb{Z})$, a direct computation show that we have

$$
\begin{array}{r}
A_{d-d^{\prime}}(1) \prod_{i=1}^{k} \prod_{\nu=1}^{d_{L_{i}}^{+}}\left(\left[L_{i}\right]+\left(d-d^{\prime}\right)_{L_{i}}+\nu\right) \prod_{\theta \in \Sigma(1)} \prod_{\nu=0}^{d_{\theta}^{+}-1}\left(\left[D_{\theta}\right]+\left(d-d^{\prime}\right)_{\theta}-\nu\right) \\
=A_{d}(1) \prod_{i=1}^{k} \prod_{\nu=1}^{d_{L_{i}}^{-}}\left(\left[L_{i}\right]+d_{L_{i}}+\nu\right) \prod_{\theta \in \Sigma(1)} \prod_{\nu=0}^{d_{\theta}^{-}-1}\left(\left[D_{\theta}\right]+d_{\theta}-\nu\right) .
\end{array}
$$

Recall that $\mathbb{G}$ is the ideal that defined $G$ (cf. Definition 4.4)

Lemma 5.21. - If $R\left(q, z, z \delta_{q}, z \delta_{z}\right)$ is in the quotient ideal Quot $\left(\widehat{c}_{\text {top }}, \mathbb{G}\right)$ then the cohomological valued function $R\left(q, z, z \delta_{q}, z \delta_{z}\right) z^{-c_{1}\left(\mathcal{T}_{X} \otimes \mathcal{E}^{\vee}\right)} z^{\mu} I(q, z)$ belongs to ker $m_{c_{\text {top }}}$ where $m_{c}$ : $\alpha \mapsto c_{\text {top }}(\mathcal{E}) \cup \alpha$.

Proof. - In this proof, we denote $d_{\mathcal{T}_{X} \otimes \mathcal{E}^{\vee}}:=\int_{d} c_{1}\left(\mathcal{T}_{X} \otimes \mathcal{E}^{\vee}\right)$. From Formulas (5.19) and (5.20), we deduce that

$$
\begin{aligned}
& R\left(q, z, z \delta_{q}, z \delta_{z}\right) q^{T+d} z^{-c_{1}\left(\mathcal{T}_{X} \otimes \mathcal{E}^{\vee}\right)-d_{\mathcal{T}_{X} \otimes \mathcal{E}^{\vee}}} \\
& =R\left(q, z, z(T+d), z\left(-c_{1}\left(\mathcal{T}_{X} \otimes \mathcal{E}^{\vee}\right)-d_{\mathcal{T}_{X} \otimes \mathcal{E}^{\vee}}\right)\right) q^{T+d} z^{-c_{1}\left(\mathcal{T}_{X} \otimes \mathcal{E}^{\vee}\right)-d_{\mathcal{T}_{X} \otimes \mathcal{E}^{\vee}}} .
\end{aligned}
$$


We decompose

$$
R\left(q, z, z \delta_{q}, z \delta_{z}\right)=\sum_{\substack{d^{\prime} \in H_{2}(X, \mathbb{Z}) \\ \text { finite }}} q^{d^{\prime}} R_{d^{\prime}}\left(z, z \delta_{q}, z \delta_{z}\right) .
$$

From Equalities (5.18) and (5.22), we deduce that

$$
R\left(q, z, z \delta_{q}, z \delta_{z}\right) z^{-c_{1}\left(\mathcal{T}_{X} \otimes \mathcal{E}^{\vee}\right)} z^{\mu} I(q, z)=\sum_{d \in H_{2}(X, \mathbb{Z})} q^{d+T} z^{-c_{1}\left(\mathcal{T}_{X} \otimes \mathcal{E}^{\vee}\right)-d_{\mathcal{T}_{X} \otimes \mathcal{E} \vee}} B_{d}(z)
$$

where

$$
B_{d}(z):=\sum_{\substack{d^{\prime} \in H_{2}(X, \mathbb{Z}) \\ \text { finite }}} R_{d^{\prime}}\left(z, z(T+d), z\left(-c_{1}\left(\mathcal{T}_{X} \otimes \mathcal{E}^{\vee}\right)-d_{\mathcal{T}_{X} \otimes \mathcal{E}^{\vee}}\right)\right) A_{d-d^{\prime}}(1) .
$$

To prove the lemme, it is enough to show that $c_{\text {top }}(\mathcal{E}) B_{d}(z)=0$ for all $d \in H_{2}(X, \mathbb{Z})$. From the definition of the ideal $\operatorname{Quot}\left(\widehat{c}_{\text {top }}, \mathbb{G}\right)$ and Lemma 5.16, we have

$$
\begin{aligned}
\widehat{c}_{\mathrm{top}} R\left(q, z, z \delta_{q}, z \delta_{z}\right) z^{-c_{1}\left(\mathcal{T}_{X} \otimes \mathcal{E}^{\vee}\right)} z^{\mu} I(q, z) & =0 \\
\sum_{d \in H_{2}(X, \mathbb{Z})} q^{d+T} z^{-c_{1}\left(\mathcal{T}_{X} \otimes \mathcal{E}^{\vee}\right)-d_{\mathcal{T}_{X} \otimes \mathcal{E} \vee}}\left(\prod_{i=1}^{k} z\left(\left[L_{i}\right]+d_{L_{i}}\right)\right) B_{d}(z) & =0 .
\end{aligned}
$$

As $c_{\text {top }}(\mathcal{E}) B_{d}: \mathbb{C} \rightarrow H^{*}(X)$ is a polynomial function in $z$, it is enough to prove that it vanishes on $\mathbb{C}^{*}$. Assume $z \in \mathbb{C}^{*}$. As $q \in\left(\mathbb{C}^{*}\right)^{r}$, we deduce that $q^{T}$ and $z^{-c_{1}\left(\mathcal{T}_{X} \otimes \mathcal{E}^{\vee}\right)}$ are invertible in $H^{*}(X)$. Denote by $I_{d}:=\left\{i \in\{1, \ldots, k\} \mid d_{L_{i}}=0\right\}$ and $I_{d}^{c}$ its complementary set. For $i \in I_{d}^{c}$, the class $\left[L_{i}\right]+d_{L_{i}}$ is invertible in $H^{*}(X)$. So we deduce that

$$
\left(\prod_{i \in I_{d}}\left[L_{i}\right]\right) B_{d}(z)=0 \text {. }
$$

This implies that $c_{\text {top }}(\mathcal{E}) B_{d}(z)=0$ as $c_{\text {top }}(\mathcal{E})=\prod_{i=1}^{k}\left[L_{i}\right]$.

Proof of Theorem 5.10. - We first prove that $\varphi$ is an isomorphism. Theorem 4.10 implies that $\operatorname{rk} \mathcal{M}=\operatorname{rk} F$. So it is enough to prove that the morphisms $\varphi$ are surjective near the large radius limit point. From (5.8) and (5.19), we deduce that for any $\alpha \in H^{2}(X)$, we have

$$
\widehat{\alpha} I(q, z)=q^{T / z}(\alpha+O(q)) .
$$

As $H^{2 *}(X)$ is generated by $H^{2}(X)$, we deduce that for any $a \in\{0, s-1\}$, there exits an operator $P_{a}\left(q, z, z \delta_{q}\right)$ (notice that we do not need $\delta_{z}$ in the operator $P_{a}$ ) such that

$$
P_{a}\left(q, z, z \delta_{q}\right) I(q, z) F(q)^{-1}=q^{T / z}\left(T_{a}+O(q)\right)
$$

where $F(q)$ is defined in Theorem 5.6. From the definition of the function $L^{\mathrm{tw}}\left(t_{0}, q, z\right)$ (cf. Equality (2.16)), we deduce that

$$
L^{\mathrm{tw}}\left(t_{0}, q, z\right) \gamma=e^{-t_{0} / z} q^{-T / z}(\gamma+O(q)) .
$$

By the mirror Theorem 5.6 we have that

$$
\operatorname{Mir}(q)=q+O(q) .
$$

Putting the last three arguments together, for any $a \in\{0, \ldots, s-1\}$ we have

$$
\varphi\left(P_{a}\left(q, z, z \delta_{q}\right)\right)=L^{\mathrm{tw}}(\operatorname{Mir}(q), z) q^{T / z}\left(T_{a}+O(q)\right)=T_{a}+o(1) .
$$

This proves the surjectivity of $\varphi$ near the large radius limit. As it is an open condition, it is true in a neighborhood of $q=0$.

Let prove that $\varphi^{\prime}$ is an isomorphism. First, the surjectivity of $\varphi$ implies the surjectivity of $\pi \circ \varphi$. We deduce that $\varphi^{\prime}$ is also surjective. On $z \neq 0$, Theorem 4.14 implies that the rank of $\mathcal{M}^{\text {res }}$ is less than $\operatorname{rk} \bar{F}$. Hence the surjectivity implies that its rank is $\operatorname{rk} \bar{F}$. This also implies 
that $\mathcal{M}^{\text {res }}$ is locally free on $U \times \mathbb{C}$ of $\operatorname{rank} \operatorname{dim}{\overline{H^{2 *}(X)}}_{\mathbb{C}}=\operatorname{rk} \bar{F}$. We deduce that $\varphi^{\prime}$ is an isomorphism.

A

\section{Twisted Axioms for Gromov-Witten invariants}

In this Appendix, we will state and prove the twisted axioms for twisted Gromov-Witten invariants. For the "untwisted" axioms, we refer to two papers of Behrend and Manin ([BM96] and [Beh97]). Some of the twisted axioms are stated (but not proved) by Pandharipande in [Pan98]. One should also mention the indirect proof given by Tseng [Tse10] where the Corollary 4.2.3 implies the twisted axioms even though there are not stated there. This appendix is due to lack of references on twisted Gromov-Witten invariants. Its aim is to fill a gap concerning results well known by experts.

Recall from Notation 2.1 and $T_{0}, \ldots, T_{s-1}$ be a basis of $H^{2 *}(X)$. We denote by $T^{a}$ the Poincaré dual of $T_{a}$ for $a \in\{0, \ldots, s-1\}$. Let $d$ be in $H_{2}(X, \mathbb{Z})$. Denote $X_{0, \ell, d}$ the moduli space of stable map of degree $d$ from rational curve with $\ell$ marked points to $X$. For $i$ in $\{1, \ldots, \ell\}$, denote by $\mathrm{e}_{i}: X_{0, \ell, d} \rightarrow X$ the evaluation map at the $i$ th marked point. The universal curve is

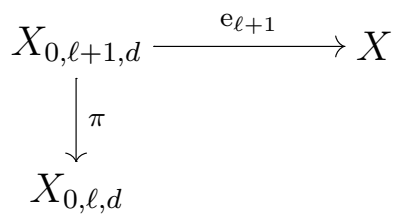

where $\pi$ is the map that forgets the $(\ell+1)$-th point and stabilizes and $\mathrm{e}_{\ell+1}$ is the evaluation at the $(\ell+1)$-th marked point. For the definition of twisted Gromov-Witten invariant, we refer to Definition 2.5. Let $\mathcal{E}_{0, \ell, d}$ be the sheaf defined in Proposition 2.2. For $j$ in $\{1, \ldots, \ell\}$, we have the following exact sequence (see (2.4))where the surjective morphism $\mathcal{E}_{0, \ell, d} \rightarrow \mathrm{e}_{j}^{*} \mathcal{E}$ evaluates the section to be the $j$-th marked point.

$$
0 \longrightarrow \mathcal{E}_{0, \ell, d}(j) \longrightarrow \mathcal{E}_{0, \ell, d} \longrightarrow \mathrm{e}_{j}^{*} \mathcal{E} \longrightarrow 0
$$

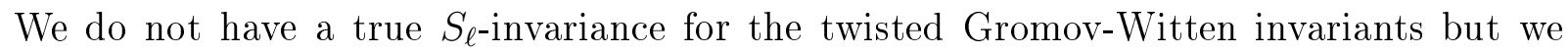
have the following proposition.

Proposition A.2 (Twisted $S_{\ell}$-invariance). - For any $\gamma_{1}, \ldots, \gamma_{\ell}$ in $H^{2 *}(X), m_{1}, \ldots, m_{\ell}$ in $\mathbb{N}$, for any $\sigma \in S_{\ell}$ and $j$ in $\{1, \ldots, \ell\}$, we have

$$
\begin{aligned}
& \left\langle\tau_{m_{1}}\left(\widetilde{c_{1}(\mathcal{E})} \cup \gamma_{1}\right), \ldots, \tau_{m_{\ell}}\left(\gamma_{\ell}\right)\right\rangle_{0, \ell, d} \\
& =\left\langle\tau_{m_{\sigma(1)}}\left(\gamma_{\sigma(1)}\right), \ldots, \tau_{m_{\sigma(j)}}\left(\widehat{c_{1}(\mathcal{E}) \cup} \gamma_{\sigma(j)}\right), \ldots, \tau_{m_{\sigma(\ell)}}\left(\gamma_{\sigma(\ell)}\right)\right\rangle_{0, \ell, d}
\end{aligned}
$$

Proof. - From the exact sequence (A.1), for any $j$ in $\{0, \ldots, \ell\}$ we have

$$
\mathrm{e}_{j}^{*}\left(c_{1}(\mathcal{E})\right) \cup c_{\text {top }}\left(\mathcal{E}_{0, \ell, d}(j)\right)=c_{\text {top }}\left(\mathcal{E}_{0, \ell, d}\right)
$$

This implies the proposition.

Let us recall some notations from Gathmann [Gat03]. For $i$ in $\{1, \ldots, \ell\}$, consider the injection morphism $\sigma_{i}: X_{0, \ell, d} \rightarrow X_{0, \ell+1, d}$ which replace the $i$-th marked point by a contracted rational component with marked point $x_{i}$ and $x_{\ell+1}$ (see Figure 2). The substack $D_{i}:=\sigma_{i}\left(X_{0, \ell, d}\right)$ is isomorphic to $X_{0, \ell, d}$ and $\pi\left(D_{i}\right)=X_{0, \ell, d}$. So $D_{i}$ carries a natural virtual fundamental class denoted by $\left[D_{i}\right]^{\mathrm{vir}}$ and it is of virtual codimension 1 . Usually, we call it boundary divisors. We have the following proposition which is proved in [Gat03]. 


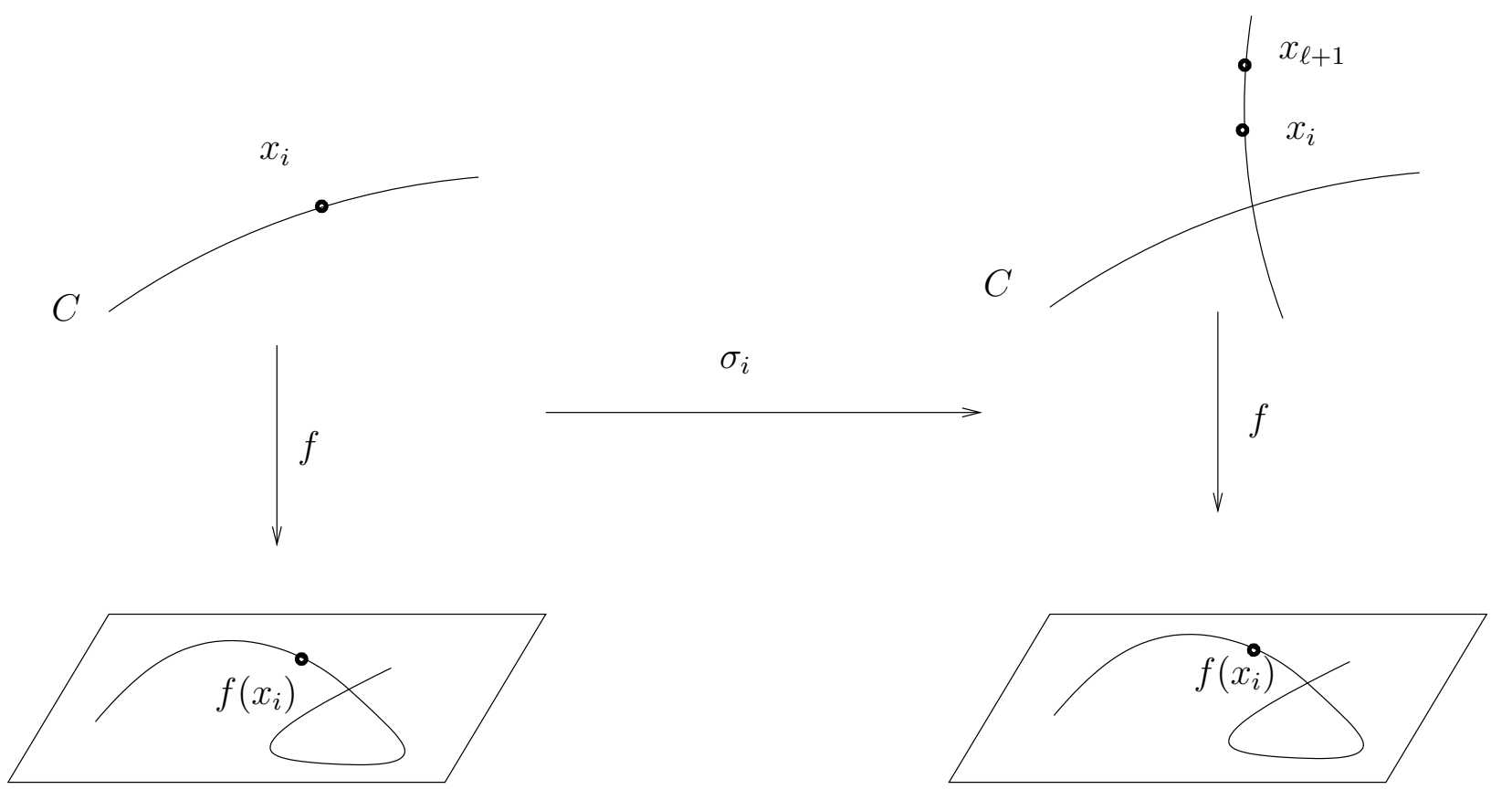

FiguRE 2. L'application $\sigma_{i}$.

Proposition A.3 (See Corollary 1.3.2 [Gat03]). - Let $\ell$ be in $\mathbb{N}$. Let d be in $H_{2}(X, \mathbb{Z})$. Let $\gamma_{1}, \ldots, \gamma_{\ell}$ be in $H^{2 *}(X)$. Let $m_{1}, \ldots, m_{\ell}$ be in $\mathbb{N}$. Let $\alpha$ be in $H^{2 *}\left(X_{0, \ell+1, d}\right)$. We have the following equality in $H^{*}\left(X_{0, \ell, d}\right)$

$$
\begin{aligned}
\pi_{*}\left(\prod_{j=1}^{\ell} \psi_{j}^{m_{j}} \mathrm{e}_{j}^{*} \gamma_{j} \cdot \alpha \cdot\left[X_{0, \ell+1, d}\right]^{\mathrm{vir}}\right) & =\prod_{j=1}^{\ell} \psi_{j}^{m_{j}} \mathrm{e}_{j}^{*} \gamma_{j} \pi_{*}\left(\alpha \cdot\left[X_{0, \ell+1, d}\right]^{\mathrm{vir}}\right) \\
& +\sum_{i \mid m_{i}>0}\left(\psi_{i}^{m_{i}-1} \mathrm{e}_{i}^{*} \gamma_{i} \prod_{\substack{j=1 \\
j \neq i}}^{\ell} \psi_{j}^{m_{j}} \mathrm{e}_{j}^{*} \gamma_{j}\right) \pi_{*}\left(\alpha \cdot\left[D_{i}\right]^{\mathrm{vir}}\right)
\end{aligned}
$$

\section{Proposition A.4 (Twisted Fundamental class equation / string equation )}

Let $\ell$ be in $\mathbb{N}, d$ be in $H_{2}(X, \mathbb{Z}), \gamma_{1}, \ldots, \gamma_{\ell}$ be in $H^{2 *}(X)$ and $m_{1}, \ldots, m_{\ell}$ be in $\mathbb{N}$. Denote by 1 the unit of the cohomology ring. For $n>2$ or $d \neq 0$ and for $k \in\{1, \ldots, n\}$, we have

$$
\begin{aligned}
& \left.\left\langle\tau_{m_{1}}\left(\gamma_{1}\right), \ldots, \widetilde{\tau_{m_{k}}\left(\gamma_{k}\right.}\right), \ldots, \tau_{m_{\ell}}\left(\gamma_{\ell}\right), \mathbf{1}\right\rangle_{0, \ell+1, d} \\
& \left.=\sum_{i \mid m_{i}>0}\left\langle\tau_{m_{1}}\left(\gamma_{1}\right), \ldots, \tau_{m_{i}-1}\left(\gamma_{i}\right), \ldots, \widetilde{\tau_{m_{k}}\left(\gamma_{k}\right.}\right), \ldots, \tau_{m_{\ell}}\left(\gamma_{\ell}\right)\right\rangle_{0, \ell, d}
\end{aligned}
$$

Remark A.5. - From Propositions A.2 and A.4, we deduce

$$
\begin{aligned}
& \left\langle\tau_{m_{1}}\left(\gamma_{1}\right), \ldots, \tau_{m_{\ell}}\left(\gamma_{\ell}\right), \widetilde{c_{1}(\mathcal{E})}\right\rangle_{0, \ell+1, d} \\
& \left.=\sum_{i: m_{i}>0}\left\langle\tau_{m_{1}}\left(\gamma_{1}\right), \ldots, \tau_{m_{i}-1} \widetilde{\left(\gamma_{i} \cup c_{1}\right.}(\mathcal{E})\right), \ldots, \tau_{m_{\ell}}\left(\gamma_{\ell}\right)\right\rangle_{0, \ell, d} \\
& =\sum_{i: m_{i}>0}\left\langle\tau_{m_{1}}\left(\widetilde{\gamma_{1} \cup c_{1}}(\mathcal{E})\right), \ldots, \tau_{m_{i}-1}\left(\gamma_{i}\right), \ldots, \tau_{m_{\ell}}\left(\gamma_{\ell}\right)\right\rangle_{0, \ell, d}
\end{aligned}
$$


Proof of Proposition A.4. - We have

$$
\begin{aligned}
& \left.\left\langle\tau_{m_{1}}\left(\gamma_{1}\right), \ldots, \widetilde{\tau_{m_{k}}\left(\gamma_{k}\right.}\right), \ldots, \tau_{m_{\ell}}\left(\gamma_{\ell}\right), \mathbf{1}\right\rangle_{0, \ell+1, d} \\
& =\operatorname{deg}\left(\pi_{*}\left(\prod_{i=1}^{\ell} \psi_{i}^{m_{i}} \mathrm{e}_{i}^{*} \gamma_{i} \cdot c_{\mathrm{top}}\left(\mathcal{E}_{0, \ell+1, d}(k)\right) \cdot\left[X_{0, \ell+1, d}\right]^{\mathrm{vir}}\right)\right)
\end{aligned}
$$

We use Proposition A.3 with $\alpha=c_{\text {top }}\left(\mathcal{E}_{0, \ell+1, d}(k)\right)$. We get

$$
\begin{aligned}
& \pi_{*}\left(\prod_{j=1}^{\ell} \psi_{j}^{m_{j}} \mathrm{e}_{j}^{*} \gamma_{j} \cdot c_{\mathrm{top}}\left(\mathcal{E}_{0, \ell+1, d}(k)\right) \cdot\left[X_{0, \ell+1, d}\right]^{\mathrm{vir}}\right) \\
& =\prod_{j=1}^{\ell} \psi_{j}^{m_{j}} \mathrm{e}_{j}^{*} \gamma_{j} \pi_{*}\left(c_{\mathrm{top}}\left(\mathcal{E}_{0, \ell+1, d}(k)\right) \cdot\left[X_{0, \ell+1, d}\right]^{\mathrm{vir}}\right) \\
& +\sum_{i \mid m_{i}>0}\left(\psi_{i}^{m_{i}-1} \mathrm{e}_{i}^{*} \gamma_{i} \prod_{\substack{j=1 \\
j \neq i}}^{\ell} \psi_{j}^{m_{j}} \mathrm{e}_{j}^{*} \gamma_{j}\right) \pi_{*}\left(c_{\mathrm{top}}\left(\mathcal{E}_{0, \ell+1, d}(k)\right) \cdot\left[D_{i}\right]^{\mathrm{vir}}\right)
\end{aligned}
$$

As $k \neq \ell+1$, we have $c_{\text {top }}\left(\mathcal{E}_{0, \ell+1, d}(k)\right)=\pi^{*} c_{\text {top }}\left(\mathcal{E}_{0, \ell, d}(k)\right)$.

By Axiom IV (See Definition 7.1) of [BM96] proved in [Beh97]. We have $\pi^{*}\left[X_{0, \ell, d}\right]$ vir $=$ $\left[X_{0, \ell+1, d}\right]^{\text {vir }}$. As $\pi$ is of relative dimension 1, the morphism $\pi_{*} \pi^{*}$ is the zero. This implies that the first term in the right hand side of (A.6) vanishes. By definition of the virtual class $\left[D_{i}\right]^{\text {vir }}$ (see paragraph before Proposition A.3), we have $\pi_{*}\left[D_{i}\right]^{\text {vir }}=\left[X_{0, l, d}\right]^{\text {vir }}$. Hence projection formula implies the proposition.

Proposition A.7 (Twisted Divisor axiom). - Let $\ell$ be in $\mathbb{N}_{\geq 0}$, $d$ be in $H_{2}(X, \mathbb{Z})$, $\gamma_{1}, \ldots, \gamma_{\ell}$ be in $H^{*}(X, \mathbb{C})$ and $m_{1}, \ldots, m_{\ell}$ be in $\mathbb{N}_{\geq 0}$. Let $\gamma$ be in $H^{2}(X, \mathbb{C})$.

$$
\begin{aligned}
& \left\langle\tau_{m_{1}}\left(\gamma_{1}\right), \ldots, \widetilde{\tau_{m_{k}}\left(\gamma_{k}\right)}, \ldots, \tau_{m_{\ell}}\left(\gamma_{\ell}\right), \gamma\right\rangle_{0, \ell+1, d} \\
& =\left(\int_{d} \gamma\right)\left\langle\tau_{m_{1}}\left(\gamma_{1}\right), \ldots, \widetilde{\tau_{m_{k}}\left(\gamma_{k}\right)}, \ldots, \tau_{m_{\ell}}\left(\gamma_{\ell}\right)\right\rangle_{0, \ell, d} \\
& \left.+\sum_{i: m_{i}>0}\left\langle\tau_{m_{1}}\left(\gamma_{1}\right), \ldots, \tau_{m_{i}-1(\gamma \cup} \gamma_{i}\right), \ldots, \tau_{m_{\ell}}\left(\gamma_{\ell}\right)\right\rangle_{0, \ell, d}
\end{aligned}
$$

Proof. - We use Proposition A.3 with $\alpha=\mathrm{e}_{\ell+1}^{*} \gamma$. We get that

$$
\begin{aligned}
& \left.\left\langle\tau_{m_{1}}\left(\gamma_{1}\right), \ldots, \widetilde{\tau_{m_{j}}\left(\gamma_{k}\right.}\right), \ldots, \tau_{m_{\ell}}\left(\gamma_{\ell}\right), \gamma\right\rangle_{0, \ell+1, d} \\
& =\operatorname{deg} \pi_{*}\left(\mathrm{e}_{\ell+1}^{*} \gamma \prod_{i=1}^{\ell} \psi_{i}^{m_{i}} \mathrm{e}_{i}^{*} \gamma_{i} \cdot c_{\mathrm{top}}\left(\mathcal{E}_{0, \ell+1, d}(k)\right) \cdot\left[X_{0, \ell+1, d}\right]^{\mathrm{vir}}\right) \\
& =\operatorname{deg}\left(\prod_{i=1}^{\ell} \psi_{i}^{m_{i}} \mathrm{e}_{i}^{*} \gamma_{i} \cdot\left[X_{0, \ell, d}\right]^{\mathrm{vir}} \cdot \pi_{*}\left(\mathrm{e}_{\ell+1}^{*} \gamma\right)\right) \\
& +\sum_{i \mid m_{i}>0} \operatorname{deg}\left(\psi_{i}^{m_{i}-1} \mathrm{e}_{i}^{*} \gamma_{i} \prod_{\substack{j=1 \\
j \neq i}} \psi_{j}^{m_{j}} \mathrm{e}_{j}^{*} \gamma_{j} \cdot \pi_{*}\left(\mathrm{e}_{\ell+1}^{*} \gamma \cdot\left[D_{i}\right]^{\mathrm{vir}}\right)\right)
\end{aligned}
$$


As $\pi$ is of relative dimension 1 , we have $\pi_{*} \mathrm{e}_{\ell+1}^{*} \gamma$ is in $A^{0}\left(X_{0, \ell, d}\right)$, we deduce that $\pi_{*} \mathrm{e}_{\ell+1}^{*} \gamma=$ $\operatorname{deg}\left(\pi_{*} \mathrm{e}_{\ell+1}^{*} \gamma \cdot[\mathrm{pt}]\right)\left[X_{0, \ell, d}\right]$. Let $[C, f, \underline{x}]$ be a point in $X_{0, \ell, d}$. By projection formula for $\pi$ and $\mathrm{e}_{\ell+1}$, we have

$$
\begin{aligned}
\operatorname{deg}\left(\pi_{*} \mathrm{e}_{\ell+1}^{*} \gamma \cdot[C, f, \underline{x}]\right) & =\operatorname{deg}\left(\pi_{*}\left(e_{\ell+1}^{*} \gamma \cdot \pi^{*}[C, f, \underline{x}]\right)\right) \\
& =\operatorname{deg}\left(e_{\ell+1}^{*} \gamma \cdot \pi^{*}[C, f, \underline{x}]\right) \\
& =\operatorname{deg}\left(\gamma \cdot \mathrm{e}_{\ell+1_{*}} \pi^{*}[C, f, \underline{x}]\right)
\end{aligned}
$$

As $\pi$ is the universal curve, $\pi^{*}[C, f, \underline{x}]$ is the class of the curve $(C, \underline{x}, f)$ and $\mathrm{e}_{\ell+1}$ restricted to this curve is $f$. So $\mathrm{e}_{\ell+1_{*}} \pi^{*}[C, f, \underline{x}]=f_{*}(C, \underline{x}, f)$ which $d$ by definition. Se we get $\operatorname{deg}\left(\pi_{*} e_{\ell+1}^{*} \gamma\right.$. $[\mathrm{pt}])=\operatorname{deg}(\gamma \cdot d)=\int_{d} \gamma$. So the term in (A.8) is exactly the first term in the right hand side of the equality of the proposition. As $\pi_{*}\left[D_{i}\right]^{\text {vir }}=\left[X_{0, \ell, d}\right]^{\text {vir }}$ and $\mathrm{e}_{\ell+1}$ is exactly $\mathrm{e}_{i}$ on $D_{i}$, projection formula implies that (A.9) is exactly the second term of the right hand side of the equality of the proposition.

Proposition A.10 (Twisted Dilaton equation). - Let $\ell$ be a non negative integer and $d$ be in $H_{2}(X, \mathbb{Z})$. Let $\gamma_{1}, \ldots, \gamma_{\ell}$ be in $H^{2 *}(X), m_{1}, \ldots, m_{\ell}$ be in $\mathbb{N}_{\geq 0}$. If $j \in\{1, \ldots, \ell\}$ we have

$$
\begin{aligned}
& \left\langle\tau_{m_{1}}\left(\gamma_{1}\right), \ldots, \widetilde{\tau_{m_{j}}\left(\gamma_{j}\right)}, \ldots, \widetilde{\tau_{m_{\ell}}\left(\gamma_{\ell}\right)}, \tau_{1}(\mathbf{1})\right\rangle_{0, \ell+1, d} \\
& =(-2+n)\left\langle\tau_{m_{1}}\left(\gamma_{1}\right), \ldots, \widetilde{\tau_{m_{j}}\left(\gamma_{j}\right)}, \ldots, \tau_{m_{\ell}}\left(\gamma_{\ell}\right)\right\rangle_{0, \ell, d}
\end{aligned}
$$

Proof. - We use Proposition A.3 with $\alpha=\psi_{\ell+1}$. We use the notation of the proof of A.7. We deduce that for a point $[C, f, \underline{x}]$ in $X_{0, \ell, d}$

$$
\pi_{*}\left(\psi_{\ell+1} \cdot \pi^{*}[C, f, \underline{x}]\right)=(-2+\ell)[C, f, \underline{x}]
$$

Changing $\mathrm{e}_{\ell+1}^{*} \gamma$ by $\psi_{\ell+1}$ in (A.8), we get

$$
\left.(-2+\ell)\left\langle\tau_{m_{1}}\left(\gamma_{1}\right), \ldots, \widetilde{\tau_{m_{j}}\left(\gamma_{j}\right.}\right), \ldots, \tau_{m_{\ell}}\left(\gamma_{\ell}\right)\right\rangle_{0, \ell, d}
$$

As the bundle $\mathfrak{N}_{\ell+1}$ (cf. before Definition 2.5) is trivial on $D_{i}$, we deduce that changing $\mathrm{e}_{\ell+1}^{*} \gamma$ by $\psi_{\ell+1}=0$ in (A.9) gives zero.

We follow Remark 1.2.8 of [Gat03]. Fix an integer $\ell$ and a homology class $d$ in $H_{2}(X, \mathbb{Z})$. Let $I_{1}, I_{2}$ be two subsets of $\{1, \ldots, \ell\}$ such that $I_{1} \sqcup I_{2}=\{1, \ldots, n\}$. Let $d_{1}, d_{2}$ in $H_{2}(X, \mathbb{Z})$ such that $d_{1}+d_{2}=d$. Denote by $\Delta: X \rightarrow X \times X$ the diagonal morphism. We define $D\left(I_{1}, d_{1} \mid I_{2}, d_{2}\right)$ by the following cartesian diagram

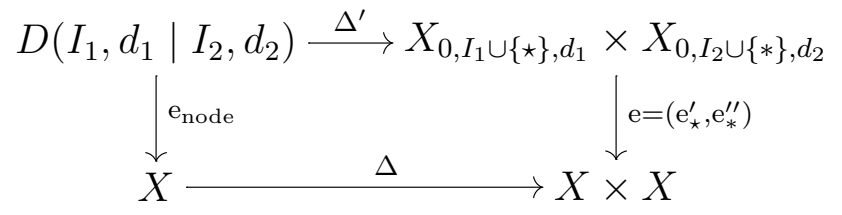

where $\mathrm{e}_{\star}^{\prime}: X_{0, I_{1}, d_{1}} \rightarrow X$ (resp. $\mathrm{e}_{*}^{\prime \prime}: X_{0, I_{2}, d_{2}} \rightarrow X$ ) is the evaluation morphism at the marked point $\star($ resp. $*)$. Geometrically, a point in the stack $D\left(I_{1}, d_{1} \mid I_{2}, d_{2}\right)$ is the data of two stable maps $\left(C_{1}, \underline{x}_{1}, f_{1}\right)$ in $X_{0, I_{1} \cup\{\star\}, d_{1}}$ and $\left(C_{2}, \underline{x}_{2}, f_{2}\right)$ in $X_{0, I_{2} \cup\{*\}, d_{2}}$ such that $f_{1}\left(x_{1, \star}\right)=f_{2}\left(x_{2, *}\right)$. Identifying the marked points $x_{1, \star}$ and $x_{2, *}$, we get a nodal curve $C=C_{1} \cup C_{2}$ with a stable map $f=\left(f_{1}, f_{2}\right): C \rightarrow X$ of degree $d=d_{1}+d_{2}$. We deduce that the $D\left(I_{1}, d_{1} \mid I_{2}, d_{2}\right) \hookrightarrow$ $X_{0, \ell, d}$ and that the map enode $: D\left(I_{1}, d_{1} \mid I_{2}, d_{2}\right) \rightarrow X$ is the evaluation at the node which is $C_{1} \cap C_{2}$. We define the virtual class on $D\left(I_{1}, d_{1} \mid I_{2}, d_{2}\right)$ by the following

$$
\left[D\left(I_{1}, d_{1} \mid I_{2}, d_{2}\right)\right]^{\mathrm{vir}}:=\Delta^{!}\left(\left[X_{0, I_{1} \cup\{\star\}, d_{1}}\right]^{\mathrm{vir}} \otimes\left[X_{0, I_{2} \cup\{*\}, d_{2}}\right]^{\mathrm{vir}}\right)
$$


A direct computation shows that the virtual codimension of $\left[D\left(I_{1}, d_{1} \mid I_{2}, d_{2}\right)\right]^{\mathrm{vir}}$ in $X_{0, \ell, d}$ is 1 that's why we call them boundary divisors ${ }^{(6)}$.

To prove twisted Topological Recursion Relations and twisted WDVV, we need to prove the twisted splitting axiom.

Proposition A.12 (Twisted splitting axiom). - Let $\ell$ be in $\mathbb{N}_{>0}$ and d be in $H_{2}(X, \mathbb{Z})$. Let $\gamma_{1}, \ldots, \gamma_{\ell}$ be in $H^{2 *}(X)$ and $m_{1}, \ldots, m_{\ell}$ be in $\mathbb{N}_{\geq 0}$. Fix a partition $I_{1} \bigsqcup I_{2}=\{1, \ldots, \ell\}$ and two homology classes $d_{1}, d_{2} \in H_{2}(X, \mathbb{C})$ such that $d_{1}+d_{2}=d$. Fix $j \in I_{1}$. Denote by $\iota$ the inclusion $D\left(I_{1}, d_{1} \mid I_{2}, d_{2}\right) \hookrightarrow X_{0, \ell, d}$. We have

$$
\begin{aligned}
& \int_{\left[D\left(I_{1}, d_{1} \mid I_{2}, d_{2}\right)\right]^{\mathrm{vir}}} c_{\mathrm{top}}\left(\iota^{*} \mathcal{E}_{0, \ell, d}(j)\right) \prod_{i=1}^{\ell} \psi_{i}^{m_{i}} \mathrm{e}_{i}^{*} \gamma_{i} \\
& =\sum_{a=0}^{s-1}\left\langle T_{a}, \widetilde{\tau_{m_{j}}\left(\gamma_{j}\right)}, \prod_{i \neq j \in I_{1}} \tau_{m_{i}}\left(\gamma_{i}\right)\right\rangle_{0, \# I_{1}+1, d_{1}}\left\langle\widetilde{T}^{a}, \prod_{i \in I_{2}} \tau_{m_{i}}\left(\gamma_{i}\right)\right\rangle_{0, \# I_{2}+1, d_{2}}
\end{aligned}
$$

Proof. - We use the notation of the diagram (A.11). Let $p_{1}: X_{0, I_{1} \cup\{\star\}, d_{1}} \times X_{0, I_{2} \cup\{*\}, d_{2}} \rightarrow$ $X_{0, I_{1} \cup\{\star\}, d_{1}}$ the projection on the first factor and $p_{2}$ the projection on the second factor. First we prove the following equality

$$
\iota^{*} c_{\text {top }}\left(\mathcal{E}_{0, \ell, d}(j)\right)=c_{\text {top }}\left(\Delta^{\prime *} p_{1}^{*} \mathcal{E}_{0, I_{1} \cup\{\star\}, d_{1}}(j) \oplus \Delta^{\prime *} p_{2}^{*} \mathcal{E}_{0, I_{2} \cup\{*\}, d_{2}}(*)\right)
$$

We define the surjective morphism $\iota^{*} \mathcal{E}_{0, \ell, d} \rightarrow \mathrm{e}_{\text {node }}^{*} \mathcal{E} \oplus \mathrm{e}_{j}^{*} \mathcal{E}$ by evaluating the section of $\iota^{*} \mathcal{E}_{0, \ell, d}$ at the $j$-th marked point and at the node ${ }^{(7)}$. We define $\mathcal{E}_{0, \ell, d}(j$, node) to be the kernel of this morphism. We deduce the following exact sequence of bundles over $D\left(I_{1}, d_{1} \mid I_{2}, d_{2}\right)$.

$$
0 \longrightarrow \mathcal{E}_{0, \ell, d}(j, \text { node }) \longrightarrow \iota^{*} \mathcal{E}_{0, \ell, d} \longrightarrow \mathrm{e}_{\text {node }}^{*} \mathcal{E} \oplus \mathrm{e}_{j}^{*} \mathcal{E} \longrightarrow 0
$$

Pulling-back the exact sequence (A.1) via the composition $p_{1} \circ \Delta^{\prime}\left(\right.$ resp. $\left.p_{2} \circ \Delta^{\prime}\right)$ on $X_{0, I_{1} \cup\{\star\}, d_{1}}$ (resp. on $X_{0, I_{2} \cup\{\star\}, d_{2}}$ ), we deduce a morphism from $\Delta^{\prime *} p_{1}^{*} \mathcal{E}_{0, I_{1} \cup\{\star\}, d_{1}}$ (resp. from $\left.\Delta^{\prime *} p_{2}^{*} \mathcal{E}_{0, I_{2} \cup\{*\}, d_{2}}\right)$ to $\mathrm{e}_{\text {node }}^{*} \mathcal{E}$. We deduce the following exact sequence

$$
0 \longrightarrow \iota^{*} \mathcal{E}_{0, \ell, d} \stackrel{a}{\longrightarrow} \Delta^{\prime *} p_{1}^{*} \mathcal{E}_{0, I_{1} \cup\{\star\}, d_{1}} \oplus \Delta^{\prime *} p_{2}^{*} \mathcal{E}_{0, I_{2} \cup\{*\}, d_{2}} \stackrel{b}{\longrightarrow} \mathrm{e}_{\text {node }}^{*} \mathcal{E} \longrightarrow 0
$$

where at the stable map $(C, \underline{x}, f)$ in $D\left(I_{1}, d_{1} \mid I_{2}, d_{2}\right)$, as $C=C_{1} \cup C_{2}$ the morphism $a$ sends a section $s \in H^{0}\left(C, f^{*} \mathcal{E}\right)$ to $\left(\left.s\right|_{C_{1}},\left.s\right|_{C_{2}}\right)$. The morphism $b$ send $\left(s_{1}, s_{2}\right)$ to $s_{1}(\star)-s_{2}(*)$. The sequence above is exact because if $s_{1}(\star)=s_{2}(*)$ then they glue in a section in $H^{0}\left(C, f^{*} \mathcal{E}\right)$. From (A.14) and (A.15), we deduce the following exact sequence

$$
0 \longrightarrow \mathcal{E}_{0, \ell, d}(j, \text { node }) \longrightarrow \Delta^{\prime *} p_{1}^{*} \mathcal{E}_{0, I_{1} \cup\{\star\}, d_{1}}(j) \oplus \Delta^{\prime *} p_{2}^{*} \mathcal{E}_{0, I_{2} \cup\{*\}, d_{2}}(*) \longrightarrow \mathrm{e}_{\text {node }}^{*} \mathcal{E} \longrightarrow 0
$$

Denote by $c_{t}(\mathcal{E})$ the total Chern class of $\mathcal{E}$. From (A.14) and (A.16), we deduce that

$$
\begin{aligned}
c_{t}\left(\Delta^{\prime *} p_{1}^{*} \mathcal{E}_{0, I_{1} \cup\{\star\}, d_{1}}(j) \oplus \Delta^{\prime *} p_{2}^{*} \mathcal{E}_{0, I_{2} \cup\{*\}, d_{2}}(*)\right) & =c_{t}\left(\mathcal{E}_{0, \ell, d}(j, \text { node })\right) c_{t}\left(\mathrm{e}_{\text {node }}^{*} \mathcal{E}\right) \\
& =c_{t}\left(\iota^{*} \mathcal{E}_{0, \ell, d}\right) c_{t}\left(\mathrm{e}_{j}^{*} \mathcal{E}\right)^{-1} \\
& =c_{t}\left(\iota^{*} \mathcal{E}_{0, \ell, d}(j)\right)
\end{aligned}
$$

This implies the Equality (A.13).

Let us prove the Equality of the Proposition A.12. Denote by $X_{1}:=X_{0, I_{1} \cup\{\star\}, d_{1}}, X_{2}:=$ $X_{0, I_{2} \cup\{*\}, d_{2}}, \mathcal{E}_{1}:=\mathcal{E}_{0, I_{1} \cup\{\star\}, d_{1}}(j)$ and $\mathcal{E}_{2}:=\mathcal{E}_{0, I_{2} \cup\{*\}, d_{2}}(*)$. We have

\footnotetext{
(6) The $D_{i}$ defined before Proposition A.3 are special cases of $D\left(I_{1}, d_{1} \mid I_{2}, d_{2}\right)$

(7) Notice that the evaluation at the node is not defined on $X_{0, \ell, d}$ but only on $D\left(I_{1}, d_{1} \mid I_{2}, d_{2}\right)$.
} 


$$
\begin{aligned}
& \int_{\left[D\left(I_{1}, d_{1} \mid I_{2}, d_{2}\right)\right]^{\mathrm{vir}}} c_{\mathrm{top}}\left(\iota^{*} \mathcal{E}_{0, \ell, d}(j)\right) \prod_{i=1}^{\ell} \psi_{i}^{m_{i}} \mathrm{e}_{i}^{*} \gamma_{i} \\
& =\operatorname{deg}\left(c_{\mathrm{top}}\left(\iota^{*} \mathcal{E}_{0, \ell, d}(j)\right) \prod_{i=1}^{\ell} \psi_{i}^{m_{i}} \mathrm{e}_{i}^{*} \gamma_{i} \Delta^{!}\left(\left[X_{1}\right]^{\mathrm{vir}} \otimes\left[X_{2}\right]^{\mathrm{vir}}\right)\right)
\end{aligned}
$$

On $X_{1}\left(\right.$ resp. $\left.X_{2}\right)$, we denote by $\psi_{i}^{\prime}\left(\right.$ resp. $\left.\psi_{i}^{\prime \prime}\right)$ the $\psi$ 's classes and $\mathrm{e}_{i}^{\prime}\left(\right.$ resp. $\left.\mathrm{e}_{i}^{\prime \prime}\right)$ the evaluation map. We put $\alpha_{i}:=\psi_{i}^{m_{i}} \mathrm{e}_{i}^{*} \gamma_{i}, \alpha_{i}^{\prime}:=\psi_{i}^{\prime m_{i}} \mathrm{e}_{i}^{\prime *} \gamma_{i}$ and $\alpha_{i}^{\prime \prime}:=\psi_{i}^{\prime \prime m_{i}} \mathrm{e}_{i}^{\prime \prime *} \gamma_{i}$. Using the Diagram (A.11) and Equality (A.13), we push forward to $X \times X$ and we get

$$
\begin{aligned}
& \int_{\left[D\left(I_{1}, d_{1} \mid I_{2}, d_{2}\right)\right]^{\mathrm{vir}}} c_{\mathrm{top}}\left(\iota^{*} \mathcal{E}_{0, \ell, d}(j)\right) \prod_{i=1}^{\ell} \alpha_{i} \\
& =\operatorname{deg}\left(\Delta_{*} \Delta^{*} \mathrm{e}_{*}\left(c_{\mathrm{top}}\left(\mathcal{E}_{1}\right) \otimes c_{\mathrm{top}}\left(\mathcal{E}_{2}\right) \cdot\left[X_{1}\right]^{\mathrm{vir}} \otimes\left[X_{2}\right]^{\mathrm{vir}} \prod_{i \in I_{1}} \alpha_{i}^{\prime} \otimes\left[X_{2}\right] \prod_{i \in I_{2}}\left[X_{1}\right] \otimes \alpha_{i}^{\prime \prime}\right)\right)
\end{aligned}
$$

Now, we use that $\Delta_{*} \Delta^{*}$ is just the intersection with the class of the diagonal, which is $\sum_{a} T_{a} \otimes T^{a}$. Using projection formula, we deduce the Equality of the proposition.

Remark A.17. - We have also the following equality

$$
\begin{aligned}
& \int_{\left[D\left(I_{1}, d_{1} \mid I_{2}, d_{2}\right)\right]^{\mathrm{vir}}} c_{\mathrm{top}}\left(\iota^{*} \mathcal{E}_{0, \ell, d}(j)\right) \prod_{i=1}^{\ell} \psi_{i}^{m_{i}} \mathrm{e}_{i}^{*} \gamma_{i} \\
& \left.=\sum_{a=0}^{s-1}\left\langle\widetilde{T_{a}} \widetilde{\tau_{m_{j}}\left(\gamma_{j}\right.}\right), \prod_{i \neq j \in I_{1}} \tau_{m_{i}}\left(\gamma_{i}\right)\right\rangle_{0, \# I_{1}+1, d_{1}}\left\langle T^{a}, \prod_{i \in I_{2}} \tau_{m_{i}}\left(\gamma_{i}\right)\right\rangle_{0, \# I_{2}+1, d_{2}}
\end{aligned}
$$

Where the double tilde mean that we are twisting in the Gromov-Witten invariant with two classes namely $c_{\text {top }}\left(\mathcal{E}_{0, I_{1} \cup\{\star\}, d_{1}}(j)\right)$ and $c_{\text {top }}\left(\mathcal{E}_{0, I_{1} \cup\{\star\}, d_{1}}(\star)\right)$. The proof is almost the same. Instead of the exact sequence (A.16), we use

$$
0 \longrightarrow \mathcal{E}_{0, \ell, d}(j, \text { node }) \longrightarrow \Delta^{* *} p_{1}^{*} \mathcal{E}_{0, I_{1} \cup\{\star\}, d_{1}}(j, \star) \oplus \Delta^{\prime *} p_{2}^{*} \mathcal{E}_{0, I_{2} \cup\{*\}, d_{2}} \longrightarrow \mathrm{e}_{\text {node }}^{*} \mathcal{E} \longrightarrow 0
$$

So we get the equality

$$
\iota^{*} c_{\text {top }}\left(\mathcal{E}_{0, \ell, d}(j)\right)=c_{\text {top }}\left(\Delta^{\prime *} p_{1}^{*} \mathcal{E}_{0, I_{1} \cup\{\star\}, d_{1}}(j, \star) \oplus \Delta^{\prime *} p_{2}^{*} \mathcal{E}_{0, I_{2} \cup\{*\}, d_{2}}\right)
$$

With the same arguments, we get Equality (A.18).

Denote by $\tau:=\sum_{a=0}^{s-1} t_{a} T_{a}$. Denote by

$$
\left\langle\left\langle\tau_{m_{1}}\left(\gamma_{1}\right), \ldots, \tau_{m_{\ell}}\left(\gamma_{\ell}\right)\right\rangle\right\rangle_{0}:=\sum_{\ell \geq 0} \sum_{d \in H_{2}(X, \mathbb{Z})} \frac{1}{\ell !}\left\langle\tau_{m_{1}}\left(\gamma_{1}\right), \ldots, \tau_{m_{\ell}}\left(\gamma_{\ell}\right), \tau, \ldots, \tau\right\rangle 0, \ell+n, d
$$

Proposition A.20 (Twisted TRR i.e., Topological Recursion Relation)

Let $\gamma_{1}, \gamma_{2}, \gamma_{3}$ be in $H^{2 *}(X)$. Let $m_{1}, m_{2}, m_{3}$ be in $\mathbb{N}_{\geq 0}$. We have the following equalities :

$$
\begin{aligned}
& \left\langle\left\langle\tau_{m_{1}+1}\left(\gamma_{1}\right), \tau_{m_{2}}\left(\gamma_{2}\right), \widetilde{\tau_{m_{3}}\left(\gamma_{3}\right)}\right\rangle\right\rangle_{0}=\sum_{a=0}^{s-1}\left\langle\left\langle\tau_{m_{2}}\left(\gamma_{2}\right), \widetilde{\tau_{m_{3}}\left(\gamma_{3}\right)}, T^{a}\right\rangle\right\rangle_{0}\left\langle\left\langle\tau_{m_{1}}\left(\gamma_{1}\right), \widetilde{T}_{a}\right\rangle\right\rangle_{0} \\
& \left\langle\left\langle\widetilde{\tau_{m_{1}+1}\left(\gamma_{1}\right)}, \tau_{m_{2}}\left(\gamma_{2}\right), \tau_{m_{3}}\left(\gamma_{3}\right)\right\rangle\right\rangle_{0}=\sum_{a=0}^{s-1}\left\langle\left\langle\tau_{m_{2}}\left(\gamma_{2}\right), \tau_{m_{3}}\left(\gamma_{3}\right), \widetilde{T}^{a}\right\rangle\right\rangle_{0}\left\langle\left\langle\widetilde{\tau_{m_{1}}\left(\gamma_{1}\right)}, T_{a}\right\rangle\right\rangle_{0}
\end{aligned}
$$


Proof. - The proof is completely parallel to the classical case ( $c f$. for instance Proposition 1.3.9 of [Gat03]). We have

$$
\psi_{1} \cdot\left[X_{0, \ell, d}\right]^{\mathrm{vir}}=\sum_{\substack{d_{1}+d_{2}=d \\ I_{1} \sqcup I_{2}=\{1, \ldots, \ell\} \\ 2,3 \in I_{1}, 1 \in I_{2}}}\left[D\left(I_{1}, d_{1} \mid I_{2}, d_{2}\right)\right]^{\mathrm{vir}}
$$

Intersecting this equality with $\psi$ 's classes and $\mathrm{e}_{i}^{*} \gamma$ and using twisted splitting axiom of Proposition A.12, we deduce the twisted TRR equality.

Remark A.23. - Using Remark A.17, we get two other twisted TRR relations

$$
\begin{aligned}
& \left.\left\langle\left\langle\tau_{m_{1}+1}\left(\gamma_{1}\right), \tau_{m_{2}}\left(\gamma_{2}\right), \widetilde{\tau_{m_{3}}\left(\gamma_{3}\right)}\right)\right\rangle\right\rangle_{0}=\sum_{a=0}^{s-1}\left\langle\left\langle\tau_{m_{2}}\left(\gamma_{2}\right), \widetilde{\tau_{m_{3}}\left(\gamma_{3}\right)}, \widetilde{T}^{a}\right\rangle\right\rangle_{0}\left\langle\left\langle\tau_{m_{1}}\left(\gamma_{1}\right), T_{a}\right)\right\rangle_{0} \\
& \left.\left\langle\left\langle\widetilde{\tau_{m_{1}+1}\left(\gamma_{1}\right)}, \tau_{m_{2}}\left(\gamma_{2}\right), \tau_{m_{3}}\left(\gamma_{3}\right)\right\rangle\right\rangle_{0}=\sum_{a=0}^{s-1}\left\langle\left\langle\tau_{m_{2}}\left(\gamma_{2}\right), \tau_{m_{3}}\left(\gamma_{3}\right), T^{a}\right\rangle\right\rangle_{0}\left\langle\widehat{\left\langle\tau_{m_{1}}\left(\gamma_{1}\right)\right.}, \widetilde{T}_{a}\right\rangle\right\rangle_{0}
\end{aligned}
$$

Proposition A.24 (Twisted WDVV equations). - Let $\gamma_{1}, \gamma_{2}, \gamma_{3}, \gamma_{4}$ be in $H^{2 *}(X)$. Let $m_{1}, m_{2}, m_{3}, m_{4}$ be in $\mathbb{N}_{\geq 0}$. We have the following equality:

$$
\begin{aligned}
& \sum_{a=0}^{s-1}\left\langle\left\langle\tau_{m_{1}}\left(\gamma_{1}\right), \tau_{m_{2}}\left(\gamma_{2}\right), \widetilde{T}_{a}\right\rangle\right\rangle_{0}\left\langle\left\langle\tau_{m_{3}}\left(\gamma_{3}\right), \widetilde{\tau_{m_{4}}\left(\gamma_{4}\right)}, T^{a}\right\rangle\right\rangle_{0} \\
& =\sum_{a=0}^{s-1}\left\langle\left\langle\tau_{m_{1}}\left(\gamma_{1}\right), \tau_{m_{3}}\left(\gamma_{3}\right), \widetilde{T}_{a}\right\rangle\right\rangle_{0}\left\langle\left\langle\tau_{m_{2}}\left(\gamma_{2}\right), \widetilde{\tau_{m_{4}}\left(\gamma_{4}\right)}, T^{a}\right\rangle\right\rangle_{0}
\end{aligned}
$$

Proof. - The proof is completely parallel to the classical case ( $c f$. for instance Proposition 1.3.8 of [Gat03]). We just use the twisted splitting axiom instead of the classical one. From Axiom V of Definition 7.1 in [BF97] proved in [Beh97], we have

$$
\sum_{\substack{d_{1}+d_{2}=d \\ I_{1} \sqcup I_{2}=\{1, \ldots, \ell\} \\ 1,2 \in I_{1}, 2,3 \in I_{2}}}\left[D\left(I_{1}, d_{1} \mid I_{2}, d_{2}\right)\right]^{\mathrm{vir}}=\sum_{\substack{d_{1}+d_{2}=d \\ I_{1} \sqcup I_{2}=\{1, \ldots, \ell\} \\ 1,3 \in I_{1}, 2,4 \in I_{2}}}\left[D\left(I_{1}, d_{1} \mid I_{2}, d_{2}\right)\right]^{\mathrm{vir}} .
$$

Intersecting this equality with $\psi$ 's classes and $\mathrm{e}_{i}^{*} \gamma$ and using twisted splitting axiom of Proposition A.12, we deduce the twisted WDVV equality.

Remark A.26. - Using the other twisted splitting axiom of Remark A.17, we get an other twisted WDVV axiom

$$
\begin{aligned}
& \sum_{a=0}^{s-1}\left\langle\left\langle\tau_{m_{1}}\left(\gamma_{1}\right), \tau_{m_{2}}\left(\gamma_{2}\right), T_{a}\right\rangle\right\rangle_{0}\left\langle\left\langle\tau_{m_{3}}\left(\gamma_{3}\right), \widetilde{\tau_{m_{4}}\left(\gamma_{4}\right)}, \widetilde{T}^{a}\right\rangle\right\rangle_{0} \\
& =\sum_{a=0}^{s-1}\left\langle\left\langle\tau_{m_{1}}\left(\gamma_{1}\right), \tau_{m_{3}}\left(\gamma_{3}\right), T_{a}\right\rangle\right\rangle_{0}\left\langle\left\langle\tau_{m_{2}}\left(\gamma_{2}\right) \widetilde{\tau_{m_{4}}\left(\gamma_{4}\right)}, \widetilde{T}^{a}\right\rangle\right\rangle_{0}
\end{aligned}
$$

B

\section{Proof of Proposition 2.17}

Proposition B.1. - 1. The connection $\nabla$ is flat. 
2. For $a \in\{1, \ldots, r\}$ and $\gamma \in H^{2 *}(X)$ we have

$$
\begin{aligned}
& \nabla_{\partial_{t_{0}}} L^{\mathrm{tw}}\left(t_{0}, q, z\right) \gamma=0, \quad \nabla_{\delta_{a}} L^{\mathrm{tw}}\left(t_{0}, q, z\right) \gamma=0 \\
& \nabla_{\delta_{z}} L^{\mathrm{tw}}\left(t_{0}, q, z\right) \gamma=L^{\mathrm{tw}}\left(t_{0}, q, z\right)\left(\mu-\frac{c_{1}\left(\mathcal{T}_{X} \otimes \mathcal{E}^{\vee}\right)}{z}\right) \gamma
\end{aligned}
$$

3. The multi-valued cohomological function $L^{\mathrm{tw}}\left(t_{0}, q, z\right) z^{-\mu} z^{c_{1}\left(\mathcal{T}_{X} \otimes \mathcal{E}^{\vee}\right)}$ is a fundamental solution of $\nabla$.

Proof. - (1) Let us prove the flatness of $\nabla$. We have to prove that for any $a, b \in\{1, \ldots, r\}$ and for any $c \in\{0, \ldots, s-1\}$, we have

$$
\begin{aligned}
& {\left[\nabla_{\delta_{a}}, \nabla_{\delta_{b}}\right] T_{c}=0} \\
& {\left[\nabla_{\delta_{a}}, \nabla_{\delta_{z}}\right] T_{c}=0}
\end{aligned}
$$

The first equation comes from the following.

$$
\begin{aligned}
& \nabla_{\delta_{a}} \nabla_{\delta_{b}} T_{c}=\frac{1}{z} \delta_{a}\left(T_{b} \bullet \bullet_{q}^{\mathrm{tw}} T_{c}\right)+\frac{1}{z^{2}}\left(T_{a} \bullet_{q}^{\mathrm{tw}}\left(T_{b} \bullet_{q}^{\mathrm{tw}} T_{c}\right)\right) \\
& \nabla_{\delta_{b}} \nabla_{\delta_{a}} T_{c}=\frac{1}{z} \delta_{b}\left(T_{a} \bullet \bullet_{q}^{\mathrm{tw}} T_{c}\right)+\frac{1}{z^{2}}\left(T_{b} \bullet_{q}^{\mathrm{tw}}\left(T_{a} \bullet_{q}^{\mathrm{tw}} T_{c}\right)\right)
\end{aligned}
$$

As $a, b \in\{1, \ldots, r\}$, the first terms are equal by the divisor axiom (see Proposition A.7). The second terms are equal by associativity and commutativity of the quantum product (see Proposition 2.14). Let us show the equation (B.3). By definition of the connection, we have

$$
\left[\nabla_{\delta_{a}}, \nabla_{\delta_{z}}\right] T_{c}=-\frac{1}{z}\left[\delta_{a}, \mathfrak{E} \bullet_{q}^{\mathrm{tw}}\right] T_{c}+\left[\frac{1}{z} T_{a} \bullet_{q}^{\mathrm{tw}}, \delta_{z}\right] T_{c}-\frac{1}{z^{2}}\left[T_{a} \bullet_{q}^{\mathrm{tw}}, \mathfrak{\bullet} \bullet_{q}^{\mathrm{tw}}\right] T_{c}+\frac{1}{z}\left[T_{a} \bullet_{q}^{\mathrm{tw}}, \mu\right] T_{c}
$$

The third term vanishes by associativity and commutativity of the quantum product. From Equalities below (B.4), (B.5), (B.6), we deduce (B.3), hence the flatness.

For any $a \in\{1, \ldots, r\}$ and for any $c \in\{0, \ldots, s-1\}$, we have

$$
\begin{aligned}
& {\left[\frac{1}{z} T_{a} \bullet_{q}^{\mathrm{tw}}, \delta_{z}\right] T_{c}=\frac{1}{z} T_{a} \bullet_{q}^{\mathrm{tw}} T_{c}} \\
& {\left[T_{a} \bullet_{q}^{\mathrm{tw}}, \mu\right] T_{c}=} \\
& \quad\left(\frac{\operatorname{deg}\left(T_{c}\right)}{2}-\operatorname{dim}_{\mathbb{C}} X\right) T_{a} \bullet_{q}^{\mathrm{tw}} T_{c}+\sum_{e=0}^{s-1} \frac{\operatorname{deg}\left(T_{e}\right)}{2} \sum_{d \in H_{2}(X, \mathbb{Z})} q^{d}\left\langle T_{a}, T_{c}, \widetilde{T}_{e}\right\rangle_{0,3, d} T^{e} \\
& \quad\left[\delta_{a}, \bullet_{q}^{\mathrm{tw}}\right] T_{c}= \\
& \quad\left(1+\frac{\operatorname{deg}\left(T_{c}\right)}{2}-\operatorname{dim}_{\mathbb{C}} X\right) T_{a} \bullet_{q}^{\mathrm{tw}} T_{c}+\sum_{e=0}^{s-1} \frac{\operatorname{deg}\left(T_{e}\right)}{2} \sum_{d \in H_{2}(X, \mathbb{Z})} q^{d}\left\langle T_{a}, T_{c}, \widetilde{T}_{e}\right\rangle_{0,3, d} T^{e}
\end{aligned}
$$

The equality (B.4) follows from

$$
\left[\frac{1}{z} T_{a} \bullet{ }_{q}^{\mathrm{tw}}, \delta_{z}\right] T_{c}=-\delta_{z}\left(\frac{1}{z} T_{a} \bullet{ }_{q}^{\mathrm{tw}}\right) T_{c}=\frac{1}{z} T_{a} \bullet \bullet_{q}^{\mathrm{tw}} T_{c} .
$$


Equality (B.5) follows from the difference of the two equalities below.

$$
\begin{aligned}
T_{a} \bullet_{q}^{\mathrm{tw}} \mu\left(T_{c}\right) & =\left(\frac{\operatorname{deg}\left(T_{c}\right)}{2}-\frac{\operatorname{dim}_{\mathbb{C}} X-\mathrm{rk} \mathcal{E}}{2}\right) T_{a} \bullet_{q}^{\mathrm{tw}} T_{c} \\
\mu\left(T_{a} \bullet_{q}^{\mathrm{tw}} T_{c}\right) & =\sum_{e=0}^{s-1} \sum_{d \in H_{2}(X, \mathbb{Z})} q^{d}\left\langle T_{a}, T_{c}, \widetilde{T}_{e}\right\rangle_{0,3, d} \mu\left(T^{e}\right) \\
& =\sum_{e=0}^{s-1}\left(\frac{\operatorname{dim}_{\mathbb{C}} X+\mathrm{rk} \mathcal{E}}{2}-\frac{\operatorname{deg} T_{e}}{2}\right) \sum_{d \in H_{2}(X, \mathbb{Z})} q^{d}\left\langle T_{a}, T_{c}, \widetilde{T}_{e}\right\rangle_{0,3, d} T^{e}
\end{aligned}
$$

(Recall that $\operatorname{deg} T^{e}=2 \operatorname{dim} X-\operatorname{deg} T_{e}$ ).

Let us prove the last equality (B.6). By Divisor Axiom A.7 and Fundamental class Axiom A.4, we have that

$$
\begin{aligned}
{\left[\delta_{a}, \mathfrak{E} \bullet{ }_{q}^{\mathrm{tw}}\right] T_{c} } & =\delta_{a}\left(\mathfrak{E} \bullet_{q}^{\mathrm{tw}} T_{c}\right) \\
& =\sum_{e=0}^{s-1} \sum_{d \in H_{2}(X, \mathbb{Z})} q^{d}\left\langle T_{a}, \mathfrak{E}, T_{c}, \widetilde{T}_{e}\right\rangle_{0,3, d} T^{e} \\
& =\sum_{e=0}^{s-1} \sum_{d \in H_{2}(X, \mathbb{Z})} q^{d} \int_{d} c_{1}\left(\mathcal{T}_{X} \otimes \mathcal{E}^{\vee}\right)\left\langle T_{a}, T_{c}, \widetilde{T}_{e}\right\rangle_{0,3, d} T^{e}
\end{aligned}
$$

Notice that if the Gromov-Witten invariant $\left\langle T_{a}, \mathfrak{E}, T_{c}, \widetilde{T}_{e}\right\rangle_{0,3, d}$ does not vanish then we have :

$$
1+\frac{\operatorname{deg}\left(T_{c}\right)}{2}+\frac{\operatorname{deg}\left(T_{e}\right)}{2}+\int_{d} c_{1}(\mathcal{E})=\int_{d} c_{1}\left(\mathcal{T}_{X}\right)+1-\operatorname{dim}_{\mathbb{C}} X
$$

so we deduce that $c_{1}\left(\mathcal{T}_{X} \otimes \mathcal{E}^{\vee}\right)=1-\operatorname{dim}_{\mathbb{C}} X+\frac{\operatorname{deg}\left(T_{c}\right)}{2}+\frac{\operatorname{deg}\left(T_{e}\right)}{2}$. Putting this in (B.7), we deduce the equality (B.6).

(2) As $\mathbf{1}$ is the unit for $\bullet_{q}^{\mathrm{tw}}$, we have the first equality.

Let us prove the second equality of (2). It is enough to prove it for $L^{\mathrm{tw}}(q, z):=L^{\mathrm{tw}}(0, q, z)$. Let $\gamma_{1}, \ldots, \gamma_{\ell} \in H^{2 *}(X)$. Denote by

$$
\left\langle\left\langle\tau_{m_{1}}\left(\gamma_{1}\right), \ldots, \tau_{m_{\ell}}\left(\gamma_{\ell}\right)\right\rangle\right\rangle_{0}^{\text {small }}
$$

the correlator defined in (A.19) where $\tau$ is replace by $\sum_{a=1}^{r} T_{a} \log q_{a}$.

Using the twisted divisor axiom (Proposition A.7) and after some computations (see [CK99], proposition 10.2.3 for example) we have :

$$
L^{\mathrm{tw}}(q, z) \gamma=\gamma-\sum_{a=0}^{s-1}\left\langle\left\langle\frac{\gamma}{z+\psi}, \widetilde{T}_{a}\right\rangle\right\rangle_{0}^{\text {small }} T^{a}
$$

where $(\psi+z)^{-1}=\sum_{\ell \geq 0}(-1)^{\ell} z^{-\ell-1} \psi^{\ell}$. For $b \in\{1, \ldots, r\}$, we have

$$
\delta_{b} L^{\mathrm{tw}}(q, z) \gamma=-\sum_{a=0}^{s-1}\left\langle\left\langle\frac{\gamma}{z+\psi}, T_{b}, \widetilde{T}_{a}\right\rangle\right\rangle_{0}^{\text {small }} T^{a}
$$

Notice that we can write the twisted quantum product with the correlator notation that is

$$
\gamma_{1} \bullet_{q}^{\mathrm{tw}} \gamma_{2}=\sum_{a=0}^{s-1}\left\langle\left\langle\gamma_{1}, \gamma_{2}, \widetilde{T}_{a}\right\rangle\right\rangle_{0}^{\mathrm{small}} T^{a}
$$


We have that

$$
\begin{aligned}
\frac{1}{z} T_{b} \bullet_{q}^{\mathrm{tw}} L^{\mathrm{tw}}(q, z) \gamma & =\frac{1}{z} \sum_{a=0}^{s-1}\left\langle\left\langle T_{b}, \gamma, \widetilde{T}_{a}\right\rangle\right\rangle_{0}^{\text {small }} T^{a} \\
& +\sum_{\ell \geq 0} \sum_{a=0}^{s-1}(-1)^{\ell+1} z^{-\ell-2} \sum_{c=0}^{s-1}\left\langle\left\langle\gamma \psi^{\ell}, \widetilde{T}_{c}\right\rangle\right\rangle_{0}^{\text {small }}\left\langle\left\langle T_{b}, T^{c}, \widetilde{T}_{a}\right\rangle\right\rangle_{0}^{\text {small }} T^{a}
\end{aligned}
$$

Using Equality (A.21) of Proposition A.20 (i.e., twisted TRR), we get

$$
\begin{aligned}
& \frac{1}{z} T_{b} \bullet{ }_{q}^{\mathrm{tw}} L^{\mathrm{tw}}(q, z) \gamma \\
& =\frac{1}{z} \sum_{a=0}^{s-1}\left\langle\left\langle T_{c}, \gamma, \widetilde{T}_{a}\right\rangle\right\rangle_{0}^{\text {small }} T^{a}+\sum_{\ell \geq 0} \sum_{a=0}^{s-1}(-1)^{\ell+1} z^{-\ell-2}\left\langle\left\langle\gamma \psi^{\ell+1}, T_{b}, \widetilde{T}_{a}\right\rangle\right\rangle_{0}^{\text {small }} T^{a} \\
& =\sum_{a=0}^{s-1}\left\langle\left\langle\frac{\gamma}{z+\psi}, T_{b}, \widetilde{T}_{a}\right\rangle\right\rangle_{0}^{\text {small }} T^{a}
\end{aligned}
$$

Adding (B.9) and (B.10), we deduce that for any $\gamma \in H^{2 *}(X)$ and any $b \in\{1, \ldots, r\}$

$$
\nabla_{\delta_{b}} L^{\mathrm{tw}}(q, z) \gamma=0
$$

To prove the last equality of (2) we use Formula (2.16) for $L^{\text {tw }}$. Then put $G^{\text {tw }}(q, z):=$ $e^{t_{0} / z} L^{\mathrm{tw}}\left(t_{0}, q, z\right) q^{T / z}$. Define the vector field $\widetilde{\mathfrak{E}}:=\sum_{a=1}^{r} e_{a} \delta_{a}+t_{0} \partial_{t_{0}}$ where $\mathfrak{E}=c_{1}\left(\mathcal{T}_{X} \otimes \mathcal{E}^{\vee}\right)=$ $\sum_{a=1}^{r} e_{a} T_{a}$. Using the second equality of (2), we have to prove that

$$
\left(z \partial_{z}+\operatorname{Lie}_{\widetilde{E}}+\mu\right) L^{\mathrm{tw}}\left(t_{0}, q, z\right) \gamma=\left(\mu-\frac{c_{1}\left(\mathcal{T}_{X} \otimes \mathcal{E}^{\vee}\right)}{z}\right) \gamma
$$

First we show that the operator $\left(z \partial_{z}+\operatorname{Lie}_{\widetilde{E}}+\mu\right)$ commutes with $G^{\mathrm{tw}}(q, z)$. Then to finish the proof we check that for any $a \in\{0, \ldots, s-1\}$ we have

$$
\begin{aligned}
\delta_{z}\left(q^{-T / z} T_{a}\right) & =z^{-1} q^{-T / z} \sum_{b=1}^{r} \log \left(q_{b}\right) T_{b} \cup T_{a} \\
\operatorname{Lie}_{\widetilde{E}}\left(q^{-T / z} T_{a}\right) & =-q^{-T / z} \frac{c_{1}\left(\mathcal{T}_{X} \otimes \mathcal{E}^{\vee}\right)}{z} T_{a} \\
\mu\left(q^{-T / z} T_{a}\right) & =q^{-T / z} \mu\left(T_{a}\right)-z^{-1} q^{-T / z} \sum_{b=1}^{r} \log \left(q_{b}\right) T_{b} \cup T_{a} \\
\left(\delta_{z}+\operatorname{Lie}_{\widetilde{E}}\right) e^{-t_{0} / z} & =0
\end{aligned}
$$

Let us prove

$$
\left(\delta_{z}+\operatorname{Lie}_{\widetilde{\mathfrak{E}}}+\mu\right) G^{\mathrm{tw}}(q, z)=G^{\mathrm{tw}}(q, z)\left(\delta_{z}+\operatorname{Lie}_{\widetilde{\mathfrak{E}}}+\mu\right) .
$$

The developing in $z$ the terms of $G^{\text {tw }}(q, z)$, we denote

$$
A(a, q, z, j, d):=z^{-j-1} q^{d}\left\langle\gamma \psi^{j}, \widetilde{T}_{a}\right\rangle_{0,2, d} T^{a}
$$

We have

$$
\begin{aligned}
\mu(A(a, q, z, j, d)) & =\left(\frac{\operatorname{dim}_{\mathbb{C}} X+\mathrm{rk} \mathcal{E}}{2}-\frac{\operatorname{deg}\left(T_{a}\right)}{2}\right) A(a, q, z, j, d) \\
\delta_{z} A(a, q, z, j, d) & =(-j-1) A(a, q, z, j, d) \\
\operatorname{Lie}_{\widetilde{E}} A(a, q, z, j, d) & =\left(\int_{d} c_{1}\left(\mathcal{T}_{X} \otimes \mathcal{E}^{\vee}\right)\right) A(a, q, z, j, d)
\end{aligned}
$$


As $A(a, q, z, j, d) \neq 0$ implies that

$$
j+\frac{\operatorname{deg}(\gamma)}{2}+\frac{\operatorname{deg}\left(T_{a}\right)}{2}+\int_{d} c_{1}(\mathcal{E})=\int_{d} c_{1}\left(\mathcal{T}_{X}\right)+2+\operatorname{dim}_{\mathbb{C}} X-3
$$

We deduce that

$$
\left(\delta_{z}+\operatorname{Lie}_{\widetilde{E}}+\mu\right) A(a, q, z, j, d)=\left(\frac{\operatorname{deg}(\gamma)}{2}-\frac{\operatorname{dim}_{\mathbb{C}} X-\operatorname{rk} \mathcal{E}}{2}\right) A(a, q, z, j, d)
$$

This implies the desired commuting relation (B.11) hence the second equality of (2).

(3) For any class $c \in H^{2}(X)$, we have that $[\mu, c]=c$. Applying the formula $\operatorname{Ad}_{\exp (X)}=e^{\operatorname{ad}_{X}}$ we deduce that $z^{\mu} \frac{c}{z} z^{-\mu}=c$. Put $c:=c_{1}\left(\mathcal{T}_{X} \otimes \mathcal{E}^{\vee}\right)$, this implies

$$
\left(\delta_{z}+\mu-\frac{c_{1}\left(\mathcal{T}_{X} \otimes \mathcal{E}^{\vee}\right)}{z}\right) z^{-\mu} z^{c_{1}\left(\mathcal{T}_{X} \otimes \mathcal{E}^{\vee}\right)}=0 .
$$

Using (2) and the equality above, for any $\gamma \in H^{2 *}(X)$, we have

$$
\begin{aligned}
& \nabla_{\delta_{z}}\left(L^{\mathrm{tw}}\left(t_{0}, q, z\right) z^{-\mu} z^{c_{1}\left(\mathcal{T}_{X} \otimes \mathcal{E}^{\vee}\right)} \gamma\right) \\
& =L^{\mathrm{tw}}\left(t_{0}, q, z\right) \delta_{z}\left(z^{-\mu} z^{c_{1}\left(\mathcal{T}_{X} \otimes \mathcal{E}^{\vee}\right)}\right)+\left(\nabla_{\delta_{z}} L^{\mathrm{tw}}\left(t_{0}, q, z\right)\right) z^{-\mu} z^{c_{1}\left(\mathcal{T}_{X} \otimes \mathcal{E}^{\vee}\right)} \gamma=0
\end{aligned}
$$

That is $L^{\mathrm{tw}}\left(t_{0}, q, z\right) z^{-\mu} z^{c_{1}\left(\mathcal{T}_{X} \otimes \mathcal{E}^{\vee}\right)}$ is a fundamental solution of $\nabla$.

\section{References}

[Ado94] Alan Adolphson, Hypergeometric functions and rings generated by monomials, Duke Math. J. 73 (1994), no. 2, 269-290. MR 1262208 (96c:33020)

[Bar00] Serguei Barannikov, Semi-infinite Hodge structures and mirror symmetry for projective spaces, Math.AG/0010157 (2000), 17.

[Bat93] Victor V Batyrev, Quantum cohomology rings of toric manifolds, alg-geom/9310004 (1993).

[Bat94] Victor V. Batyrev, Dual polyhedra and mirror symmetry for Calabi-Yau hypersurfaces in toric varieties, J. Algebraic Geom. 3 (1994), no. 3, 493-535. MR 1269718 (95c:14046)

[Beh97] K. Behrend, Gromov-Witten invariants in algebraic geometry, Invent. Math. 127 (1997), no. $3,601-617$.

[BF97] K. Behrend and B. Fantechi, The intrinsic normal cone, Invent. Math. 128 (1997), no. 1, 45-88.

[BH93] Winfried Bruns and Jürgen Herzog, Cohen-Macaulay rings, Cambridge Studies in Advanced Mathematics, vol. 39, Cambridge University Press, Cambridge, 1993.

[BM96] K. Behrend and Yu. Manin, Stacks of stable maps and Gromov-Witten invariants, Duke Math. J. 85 (1996), no. 1, 1-60.

[CCLT06] Tom Coates, Alessio Corti, Y.-P. Lee, and Hsian-Hua Tseng, Small quantum orbifold cohomology of weighted projective spaces, math.AG/0608481 (2006), 50.

[CG07] Tom Coates and Alexander Givental, Quantum Riemann-Roch, Lefschetz and Serre, Ann. of Math. (2) $\mathbf{1 6 5}$ (2007), no. 1, 15-53.

[CK99] David A. Cox and Sheldon Katz, Mirror symmetry and algebraic geometry, Mathematical Surveys and Monographs, vol. 68, American Mathematical Society, Providence, RI, 1999.

[CLS11] David Cox, John Little, and Hal Schenck, Toric varieties, http://www.cs.amherst.edu/ dac/toric.html (2011).

[CvR08] David A Cox and Christine von Renesse, Primitive collections and toric varieties, 0808.1836 (2008).

[Eis95] David Eisenbud, Commutative algebra, Graduate Texts in Mathematics, vol. 150, SpringerVerlag, New York, 1995, With a view toward algebraic geometry. 
[FP97] W. Fulton and R. Pandharipande, Notes on stable maps and quantum cohomology, Algebraic geometry-Santa Cruz 1995, Proc. Sympos. Pure Math., vol. 62, Amer. Math. Soc., Providence, RI, 1997, pp. 45-96.

[Fu193] William Fulton, Introduction to toric varieties, Annals of Mathematics Studies, vol. 131, Princeton University Press, Princeton, NJ, 1993, The William H. Roever Lectures in Geometry.

[Gat03] Andreas Gathmann, Gromov-Witten invariants of hypersurfaces, Habilitation thesis (2003), http://www.mathematik.uni-kl.de/ gathmann/pub.php.

[GGZ87] I. M. Gel'fand, M. I. Graev, and A. V. Zelevinskiı̌, Holonomic systems of equations and series of hypergeometric type, Dokl. Akad. Nauk SSSR 295 (1987), no. 1, 14-19.

[Giv95] A. B. Givental', Homological geometry. I. Projective hypersurfaces, Selecta Math. (N.S.) 1 (1995), no. 2, 325-345.

[Giv96] Alexander B. Givental, Equivariant Gromov-Witten invariants, Internat. Math. Res. Notices (1996), no. 13, 613-663, alg-geom/9603021.

[Giv98] Alexander Givental, A mirror theorem for toric complete intersections, Topological field theory, primitive forms and related topics (Kyoto, 1996), Progr. Math., vol. 160, Birkhäuser Boston, Boston, MA, 1998, pp. 141-175.

[GKZ90] I. M. Gel'fand, M. M. Kapranov, and A. V. Zelevinsky, Generalized Euler integrals and A-hypergeometric functions, Adv. Math. 84 (1990), no. 2, 255-271.

[Gro66] A. Grothendieck, Éléments de géométrie algébrique. IV: Étude locale des schemas et des morphismes de schemas. (Troisieme partie). (French).

[GS08] M. A. Guest and H. Sakai, Orbifold quantum D-modules associated to weighted projective spaces, ArXiv e-prints (2008).

[Gue10] Martin A. Guest, Differential equations aspects of quantum cohomology, Geometric and topological methods for quantum field theory, Cambridge Univ. Press, Cambridge, 2010, pp. 5485.

[GZK88] I. M. Gel'fand, A. V. Zelevinskiŭ, and M. M. Kapranov, Equations of hypergeometric type and Newton polyhedra, Dokl. Akad. Nauk SSSR 300 (1988), no. 3, 529-534.

[GZK89] _ Hypergeometric functions and toric varieties, Funktsional. Anal. i Prilozhen. 23 (1989), no. 2, 12-26.

[Her06] Claus Hertling, $t t^{*}$ geometry and mixed Hodge structures, Singularity theory and its applications, Adv. Stud. Pure Math., vol. 43, Math. Soc. Japan, Tokyo, 2006, pp. 73-84.

[HTT08] Ryoshi Hotta, Kiyoshi Takeuchi, and Toshiyuki Tanisaki, D-modules, perverse sheaves, and representation theory, Progress in Mathematics, vol. 236, Birkhäuser Boston Inc., Boston, MA, 2008, Translated from the 1995 Japanese edition by Takeuchi.

[Iri06] Hiroshi Iritani, Quantum D-modules and equivariant Floer theory for free loop spaces, Math. Z. 252 (2006), no. 3, 577-622.

[Iri07] _ Convergence of quantum cohomology by quantum Lefschetz, J. Reine Angew. Math. 610 (2007), 29-69.

[Iri08] , Quantum D-modules and generalized mirror transformations, Topology 47 (2008), no. 4, 225-276.

[Iri09] _ An integral structure in quantum cohomology and mirror symmetry for toric orbifolds, Adv. Math. 222 (2009), no. 3, 1016-1079.

[Iri11] Hiroshi Iritani, Quantum cohomology and periods, 1101.4512 (2011).

[Kar78] Max Karoubi, K-theory, Springer-Verlag, Berlin, 1978, An introduction, Grundlehren der Mathematischen Wissenschaften, Band 226.

[Kim99] Bumsig Kim, Quantum hyperplane section theorem for homogeneous spaces, Acta Math. 183 (1999), no. 1, 71-99. MR 1719555 (2001i:14076)

[KKP03] Bumsig Kim, Andrew Kresch, and Tony Pantev, Functoriality in intersection theory and a conjecture of Cox, Katz, and Lee, J. Pure Appl. Algebra 179 (2003), no. 1-2, 127-136.

[KKP08] L. Katzarkov, M. Kontsevich, and T. Pantev, Hodge theoretic aspects of mirror symmetry, ArXiv e-prints (2008). 
[Kon95] Maxim Kontsevich, Homological algebra of mirror symmetry, Proceedings of the International Congress of Mathematicians, Vol. 1, 2 (Zürich, 1994) (Basel), Birkhäuser, 1995, pp. 120-139. MR 1403918 (97f:32040)

[Mat86] Hideyuki Matsumura, Commutative ring theory, Cambridge Studies in Advanced Mathematics, vol. 8, Cambridge University Press, Cambridge, 1986, Translated from the Japanese by M. Reid.

[Mav00] Anvar R Mavlyutov, On the chiral ring of Calabi-Yau hypersurfaces in toric varieties, math/0010318 (2000).

[Mus] Mircea Mustata, Toric varieties.

[Pan98] Rahul Pandharipande, Rational curves on hypersurfaces (after A. Givental), Astérisque (1998), no. 252, Exp. No. 848, 5, 307-340, Séminaire Bourbaki. Vol. 1997/98, math/9806133.

[RS10] T. Reichelt and C. Sevenheck, Logarithmic Frobenius manifolds, hypergeometric systems and quantum D-modules, arXiv:1010.2118 (2010).

[Sab05] Claude Sabbah, Polarizable twistor D-modules, Astérisque (2005), no. 300, vi +208.

[Stu96] Bernd Sturmfels, Gröbner bases and convex polytopes, University Lecture Series, vol. 8, American Mathematical Society, Providence, RI, 1996.

[Tse10] Hsian-Hua Tseng, Orbifold quantum Riemann-Roch, lefschetz and serre, Geometry and Topology (2010), no. 14, 1-81, math/0506111.

Etienne Mann, Etienne Mann, Université Montpellier 2, Institut de Mathématique et de Modélisation de Montpellier, UMR 5149, Case courier 051 Place Eugène Bataillon F-34 095 Montpellier CEDEX 5 • E-mail : etienne.mann@math.univ-montp2.fr Url : http://www.math.univ-montp2.fr/ ${ }^{\sim} \operatorname{mann} /$ index.html

Thierry Mignon, Thierry Mignon, Université Montpellier 2, Institut de Mathématique et de Modélisation de Montpellier, UMR 5149, Case courier 051 Place Eugène Bataillon F-34 095 Montpellier CEDEX 5 • E-mail : thierry.mignon@math.univ-montp2.fr Url : http://www.math.univ-montp2.fr/ 〜mignon/ 